\title{
MODOPTIM: A General Optimization Program for Ground-Water Flow Model Calibration and Ground-Water Management with MODFLOW
}
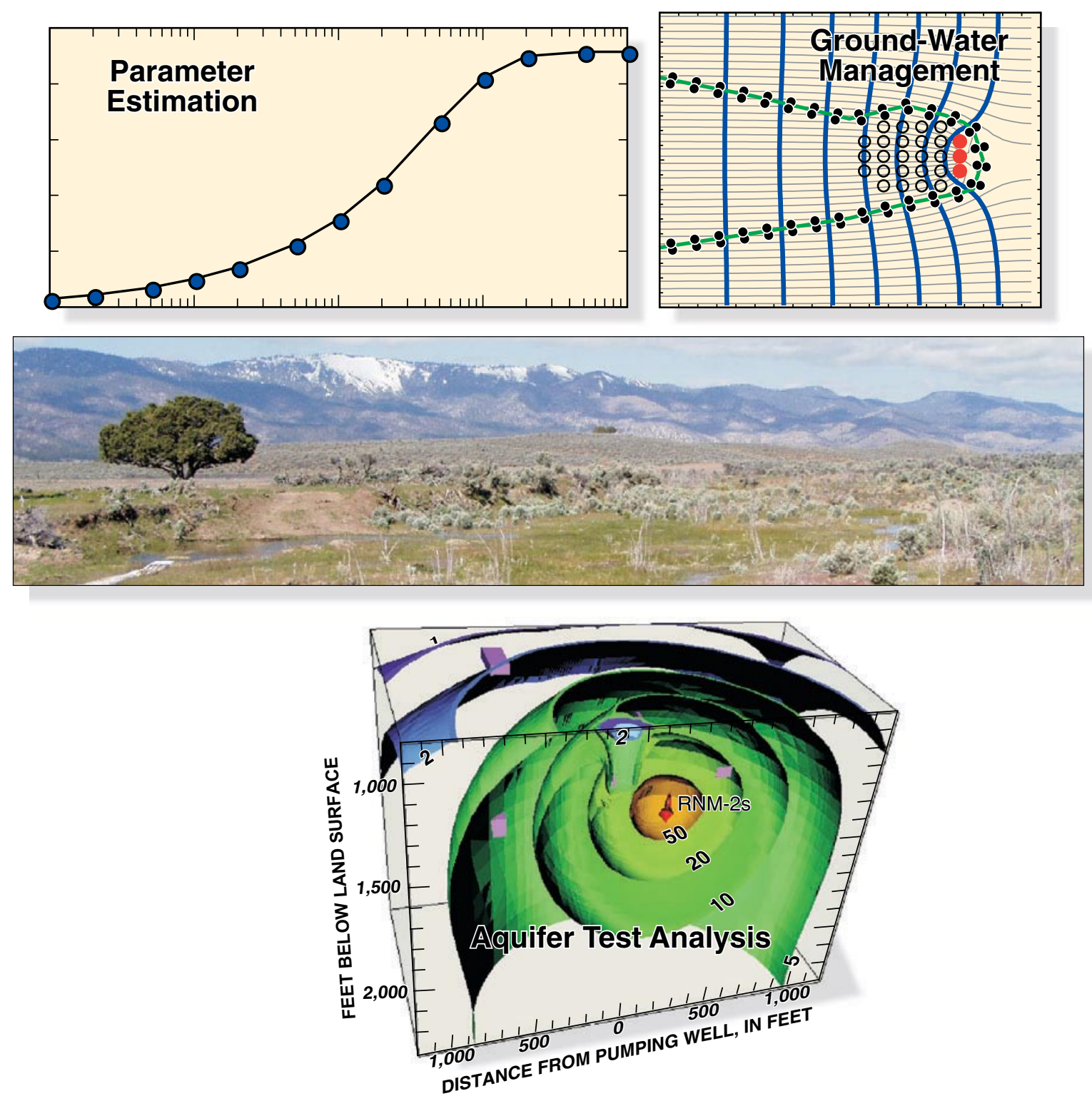

Scientific Investigations Report 2006-5009

U.S. Department of the Interior

U.S. Geological Survey 



\section{MODOPTIM: A General Optimization Program for Ground- Water Flow Model Calibration and Ground-Water Management with MODFLOW}

By Keith J. Halford

U.S. GEOLOGICAL SURVEY

Scientific Investigations Report 2006-5009

Carson City, Nevada

2006 


\title{
U.S. DEPARTMENT OF THE INTERIOR
}

\author{
P. LYNN SCARLETT, Acting Secretary
}

\section{U.S. GEOLOGICAL SURVEY}

P. Patrick Leahy, Acting Director

Any use of trade, product, or firm names in this publication is for descriptive purposes only and does not imply endorsement by the U.S. Government.

For additional information write to:

U.S. Geological Survey

Director, USGS Nevada Integrated Science Center

333 West Nye Lane, Room 203

Carson City, NV 89706-0866

Email: GS-W-NVpublic-info@usgs.gov

URL: http://nevada.usgs.gov/ 


\section{CONTENTS}

Abstract

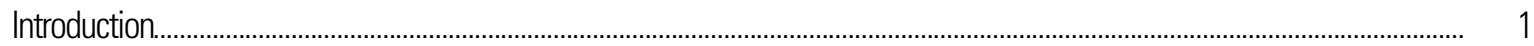

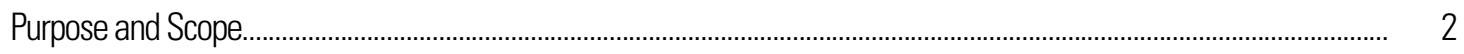

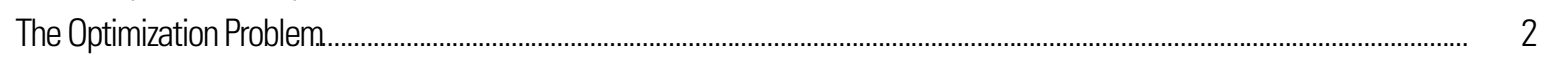

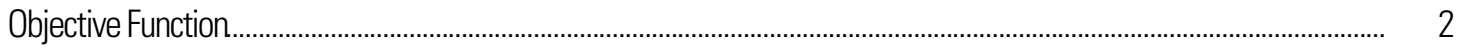

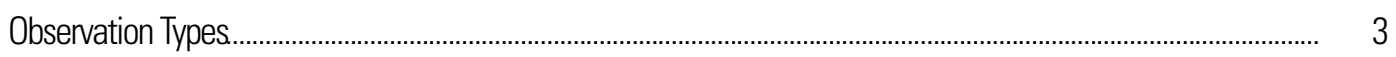

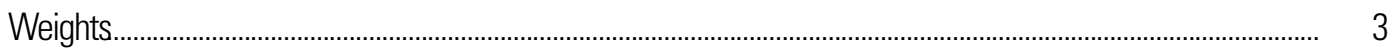

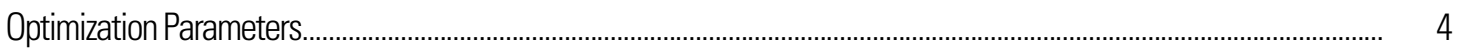

Zonation and Parameter-Weight Matrices............................................................................................................. 4

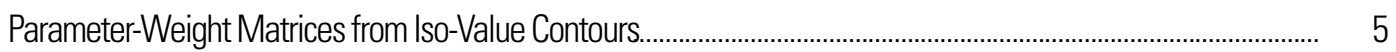

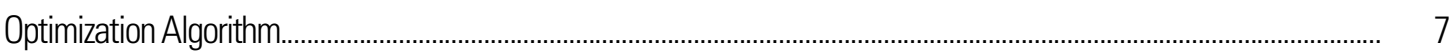

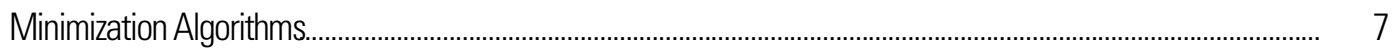

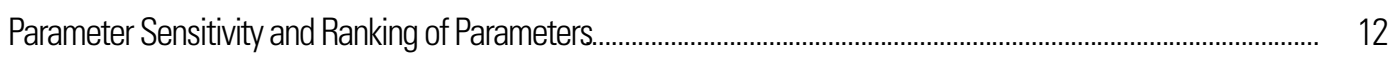

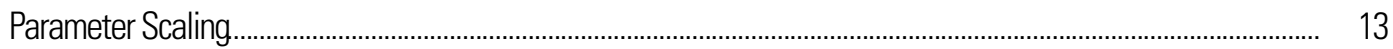

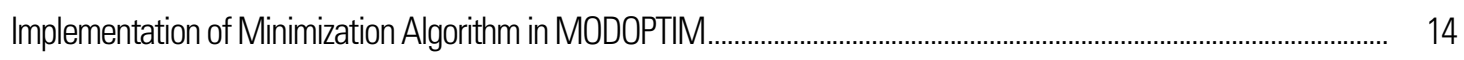

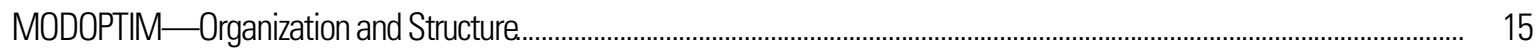

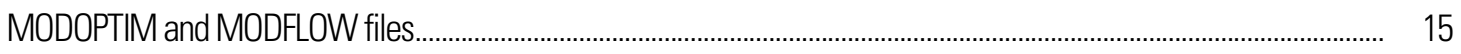

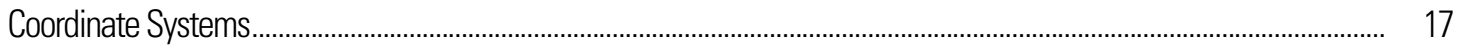

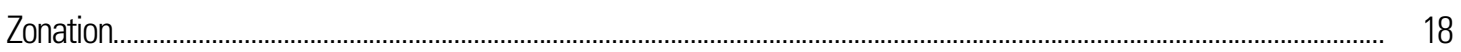

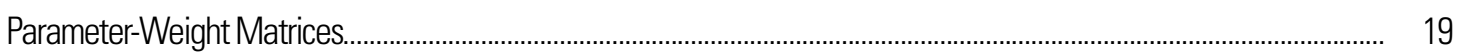

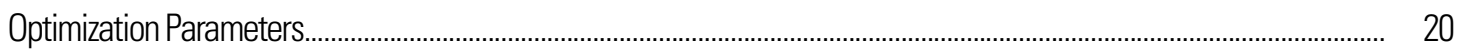

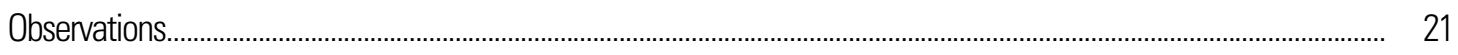

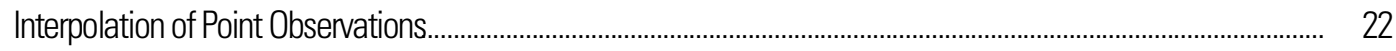

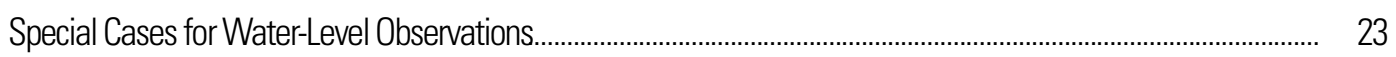

Water-Levels in Pumping Wells....................................................................................................................... 23

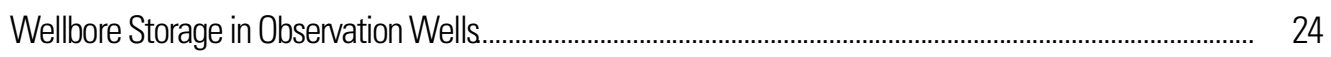

Rotation of Observation Wells................................................................................................................... 24

Subsidence Observations ..................................................................................................................................... 25

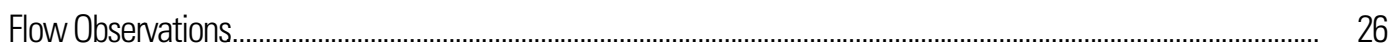

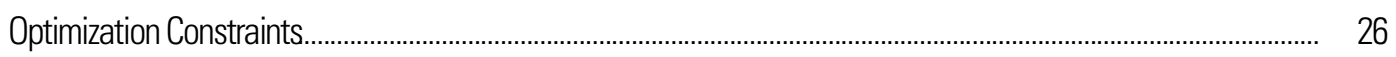

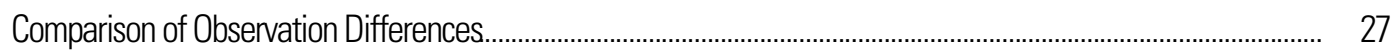


Weighting of Observations.................................................................................................................................................. 28

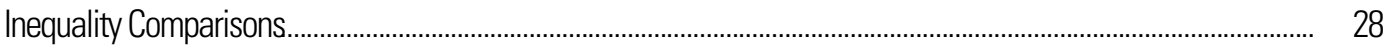

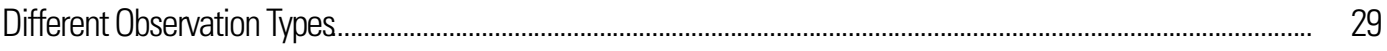

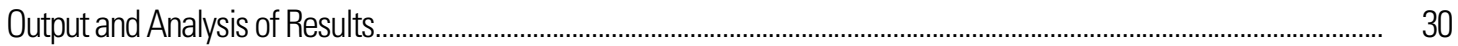

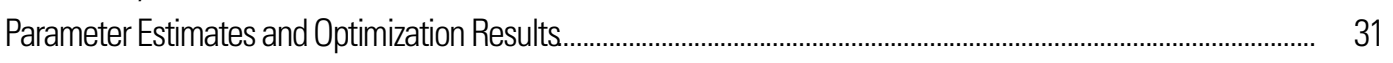

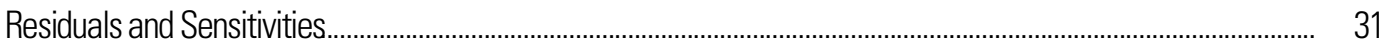

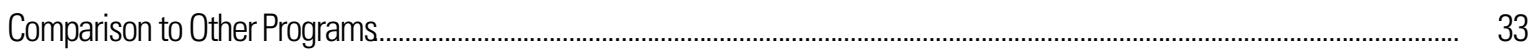

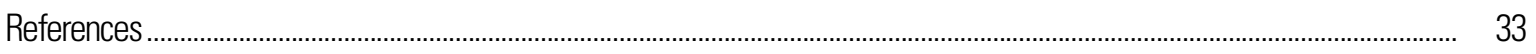

Appendix A Input structures used in MODOPTIM files ....................................................................... 36

Appendix B Input for main optimization file ………............................................................................... 38

Appendix C Input for composite MODFLOW/MODOPTIM file with parameters to be estimated ............... 48

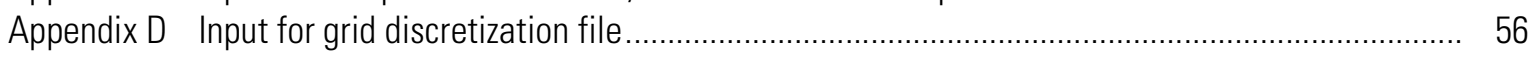

Appendix E Time-variant hydraulic-property package (VAR1) ....................................................... 59 


\section{FIGURES}

Figure 1. Two hypothetical hydraulic-conductivity distributions: (a) zones and (b) a parameter-weight matrix.............. 5

Figure 2. An iso-value contour imposed on a 10-by-10 grid and the zone of influence for a segment of the isovalue contour.

Figure 3. Two hydraulic-conductivity distributions constructed with a parameter-weight matrix of distances to the iso-value contour depicted in figure 2 .....

Figure 4. A two-parameter error surface and two steepest-descent search paths. ................................................... 7

Figure 5. A steepest-descent and a Gauss-Newton search path.............................................................. 9

Figure 6. The root-mean-square error change of the search paths shown in figure 5.......................................... 9

Figure 7. Errors in search direction and range from the Gauss-Newton approximation............................................ 10

Figure 8. Effect of the variable a on the direction and magnitude of parameter change. .......................................... 11

Figure 9. Comparison of the relative sensitivity of natural recharge, vertical anisotropy, and underflow recharge to root-mean-square error change as these same parameters are varied independently ............................... 12

Figure 10. Effect of log transforms and scaling on parameter sensitivity .............................................................. 15

Figure 11. Flow chart of MODOPTIM main program. …….................................................................................... 16

Figure 12. Relation between Cartesian coordinate system and layer, row, and column indices that are used to locate observation points, define zones, and construct weight matrices from iso-value lines.................... 17

Figure 13. Example of an IZONE array constructed from three polygons.............................................................. 18

Figure 14. Effect of weight matrices WM1 and WM2 on the spatial variability of a hydraulic property distribution and the temporal variability of a pumpage distribution, respectively ....................................... 19

Figure 15. A recharge distribution from two optimization parameters that share a common zone. .............................. 21

Figure 16. Bi-linear interpolation from the four surrounding nodes in a layer and tri-linear interpolation from the eight surrounding nodes to a point observation.............................................................................. 22

Figure 17. Interpretation of head change between layers in a quasi-3D and 3D simulation. .................................... 23

Figure 18. Rotation of lateral transmissivity ellipse or rotation of observation wells about the production well

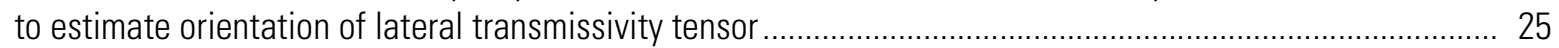

Figure 19. Translation of observation wells to a quarter section........................................................................... 25

Figure 20. Discharge from river and drain cells that are summed over an area that spans two observation zones........... 26

Figure 21. Effect of comparing water levels and water levels from an offset time on sensitivity estimates.................... 27

Figure 22. Error response to minimum and maximum ground-water discharge estimates as (a) two independent measurements and (b) a pair of inequality measurements ................................................................ 29

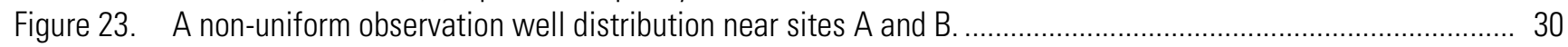

Figure 24. Weighted sensitivities for transmissivity change in a Theis aquifer at radii of 25, 100, and 400 feet

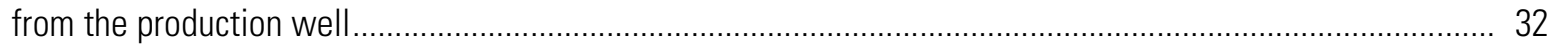

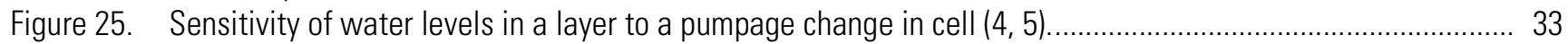




\section{CONVERSION FACTORS AND ABBREVIATED WATER-QUALITY UNITS}

\begin{tabular}{rll}
\hline Multiply & \multicolumn{1}{c}{ By } & To obtain \\
\hline foot $(\mathrm{ft})$ & 0.3048 & meter \\
foot squared per day $1\left(\mathrm{ft}^{2} / \mathrm{d}\right)$ & 0.0929 & meter squared per day \\
cubic foot per second $\left(\mathrm{ft}^{3} / \mathrm{s}\right)$ & 0.02832 & cubic meter per second \\
gallon per minute $(\mathrm{gal} / \mathrm{min})$ & 0.06308 & liter per second \\
\hline
\end{tabular}

${ }^{1}$ Expresses transmissivity. An alternative way of expressing transmissivity is cubic foot per day per square foot, times foot of aquifer thickness.

Chemical concentration is discussed in metric units, in milligrams per liter (mg/L). Milligrams per liter is a unit expressing the mass of solute (milligrams) per unit volume (liter) of water. One thousand micrograms per liter is equivalent to 1 milligram per liter. For concentrations less than 7,000 milligrams per liter, the numerical value is about the same as for concentrations in parts per million. 


\title{
MODOPTIM: A General Optimization Program for Ground-Water Flow Model Calibration and Ground-Water Management with MODFLOW
}

\author{
By Keith J. Halford
}

\section{ABSTRACT}

MODOPTIM is a non-linear ground-water model calibration and management tool that simulates flow with MODFLOW-96 as a subroutine. A weighted sum-of-squares objective function defines optimal solutions for calibration and management problems. Water levels, discharges, water quality, subsidence, and pumping-lift costs are the five direct observation types that can be compared in MODOPTIM. Differences between direct observations of the same type can be compared to fit temporal changes and spatial gradients. Water levels in pumping wells, wellbore storage in the observation wells, and rotational translation of observation wells also can be compared. Negative and positive residuals can be weighted unequally so inequality constraints such as maximum chloride concentrations or minimum water levels can be incorporated in the objective function. Optimization parameters are defined with zones and parameter-weight matrices. Parameter change is estimated iteratively with a quasi-Newton algorithm and is constrained to a user-defined maximum parameter change per iteration. Parameters that are less sensitive than a userdefined threshold are not estimated. MODOPTIM facilitates testing more conceptual models by expediting calibration of each conceptual model. Examples of applying MODOPTIM to aquifer-test analysis, ground-water management, and parameter estimation problems are presented.

\section{INTRODUCTION}

Ground-water model calibration and ground-water management problems seek to determine the "best" estimates of model parameters. "Best" estimates of hydraulic conductivity and recharge distributions are determined by minimizing differences between simulated and measured observations such as water levels and discharges for model calibration problems. Withdrawal and injection schedules are optimized by minimizing differences between simulated and desired conditions such as water levels, water-level gradients, water-level declines, flow rates, water quality, and pumping-lift costs for ground-water management problems.

Ground-water model calibration and ground-water management evaluations can be described collectively as optimization problems. The theory and application of optimization methods to the simulation of fluid flow in porous media has been documented by many investigators in both the hydrologic sciences and petroleum engineering. Optimization terminology includes many names, such as parameter estimation (Townley and Wilson, 1985; Carrera and Neuman, 1986), nonlinear regression (Cooley, 1982; Vecchia and Cooley, 1987; Hill, 1992), inverse problem (Kuiper, 1986), automatic calibration (Holmes and others, 1989; Poeter and Hill, 1997), automatic history-matching (Jacquard and Jain, 1965; Jahns, 1966; Coats and others, 1970; Thomas and others, 1971; Chen and others, 1974; Watson and Lee, 1986; Yang and others, 1987), optimal control (Chavent and others, 1975; Wasserman and others, 1975), optimal ground-water management (Gorelick, 1983; Dougherty and Marryott, 1991; Chau, 1992), systems analysis (Yeh, 1992), and optimization (Gorelick and Wagner, 1986; Reichard, 1995).

Optimization techniques are a superior method for determining unknown model parameters than trial-and-error methods. Optimization forces a modeler to carefully examine and rigorously define which observations must be simulated well to achieve a "best" fit. The relative importance or sensitivity of each parameter and degree of correlation between parameters can be quantitatively assessed within the context of how a modeler has defined "best." Optimization yields better estimates of model parameters than trial-and-error methods because, unlike human modelers, an algorithm is indifferent to the inherent tedium of model calibration. 
MODOPTIM is an optimization tool that facilitates calibrating ground-water flow models and managing ground-water development. Addressing both calibration and management problems is needed because the relative worth of a ground-water management solution is dependent on how well the underlying ground-water flow model is calibrated. Combining both functions in one tool eases the transition from model calibration to ground-water management and reduces the learning curve for users.

\section{Purpose and Scope}

This report describes the organization, structure, and use of the computer program MODOPTIM. The theory and implementation of the optimization algorithm also are described. Data input, model output, and general applications of MODOPTIM are illustrated with examples of aquifer-test analysis, ground-water management, and parameter estimation.

\section{THE OPTIMIZATION PROBLEM}

Application of optimization methods to the simulation of fluid flow in porous media has three basic components regardless of the specific problem being solved. The "best" solution must be formally defined by an objective function that provides the basis of comparison between simulated and measured observations. The unknowns to be estimated such as aquifer properties or well-pumping schedules must be well-posed parameters. An optimization technique is needed that will minimize differences between measured and simulated observations.

\section{Objective Function}

The objective function defines what will be optimized and is a measure of how well a ground-water flow model is calibrated or a ground-water management solution meets the desired goals. The reasonableness of parameter estimates is highly dependent on how the objective function is formulated. If the objective function is poorly formulated, the optimization algorithms may reduce the difference between simulated and measured observations to nothing but will produce meaningless parameter estimates.

Model error is defined by a weighted, sum-of-squares objective function in MODOPTIM.

$$
\begin{gathered}
S S(x)=\sum_{i=1}^{n o b s} f(x)_{i}^{2} \\
f(x)_{i}=\left(\hat{o}(x)_{i}-o_{i}\right) w_{i}
\end{gathered}
$$

where:

$x \quad$ is the vector of parameters being estimated,

nobs is the number of simulated and measured observations that are compared, and

$f(x)_{i} \quad$ is the $i^{\text {th }}$ weighted residual which is the difference between the $i^{\text {th }}$ simulated observation $\left(\hat{o}(x)_{i}\right)$ and the $i^{\text {th }}$ measured observation $\left(o_{i}\right)$ times the $i^{\text {th }}$ weight $\left(w_{i}\right)$. The weights emphasize more accurate measurements, allow for the use of multiple measurement types with different units, and facilitate incorporating inequality comparisons.

Although the sum-of-squares serves as the objective function, root-mean-square (RMS) error is frequently reported instead because RMS error is more directly comparable to actual values and serves as a composite of the average and the standard deviation of a set of compared values. Root-mean-square error is related to the sum-ofsquares error by 


\section{Observation Types}

$$
R M S=\sqrt{\frac{S S}{n o b s}} .
$$

Water levels, flows, water quality, subsidence, and pumping-lift costs are the five direct observation types that can be compared in MODOPTIM. A wide range of observation types are available to accommodate both model calibration and ground-water management. Water levels and discharges are the direct observation types used for model calibration. For ground-water management problems, water-quality targets, such as chloride or total-dissolvedsolids concentrations, and pumping-lift costs are additional observation types that describe management objectives and constrain parameter estimates. The additional concentration and reference elevation information needed to simulate flow-rate-averaged concentrations and pumping-lift costs, respectively, are acquired and tracked within MODFLOW by the multi-node-well (MNW) package (Halford and Hanson, 2002).

A change or difference between two direct observations of the same type can serve as a derived type of observation. Differences between water levels in a cluster of wells, incremental gains or losses along a river reach, water-level declines in a pumped well, and seasonal water-level changes in an observation well that lacks vertical geodetic control are examples of derived observation types that might constrain model calibration. Derived observation types also are needed for ground-water management problems. Controlling the advective movement of contaminants, minimizing water-level declines, and pumping-lift costs are a few examples of ground-water management constraints that are better posed as derived observation types.

Water levels in pumping wells and water levels affected by wellbore storage in observation wells also can be compared using MODOPTIM. The effects of these two special cases are normally neglected unless the model is discretized very finely around the production or observation wells of interest (Barrash and Dougherty, 1997). Water levels in pumping wells are approximated by simulating the head difference between a model cell and the well using the Theim equation, which assumes that steady-state flow occurs near the pumping well. Water-level responses in an observation well can lag because the storage coefficient of a wellbore is great relative to the surrounding aquifer. Wellbore storage effects in observation wells generally are not significant except for the analysis of some aquifer tests. Neglecting wellbore storage in the observation wells can cause the specific storage of an aquifer to be overestimated (Moench, 1997).

\section{Weights}

Different observation types are made comparable by the use of weights and composited into a single objective function (Watson and others, 1980; Cooley, 1982; Carrera and Neuman, 1986; Hill, 1992). Weights account for differences in units and normalize the relative sensitivity of the optimization routine to observations of different types that share the same units. For example, the range of water-level differences between aquifers can be substantially smaller than the range of water-level measurements within aquifers. Consequently, one might want to weight waterlevel differences more than water-level measurements if the relative importance of the two observation types is perceived as being similar.

If disparate types of observations are not formally combined into one objective function, the individual calibrating a model or solving a ground-water management problem will still subjectively weight the importance of minimizing one error term more than another. A composite objective function does not remove this subjectivity. Defining an objective function formalizes the problem and forces the individual calibrating a model to consciously acknowledge the relative importance of matching different observation types such as water levels, water-level differences, and stream-discharge measurements.

Inequality comparisons or one-sided constraints can be incorporated in the objective function by unequal weighting of residuals. Two weights are specified for each observation, one for positive residuals and the other for negative residuals. An inequality comparison is specified by assigning a weight of 0 to the unconstrained residuals. For example, a chloride concentration target of $250 \mathrm{mg} / \mathrm{L}$ would be specified, a relatively large weight would be assigned to positive residuals, and a weight of 0 would be assigned to negative residuals. Positive residuals occur where simulated concentrations are greater than target concentrations. 
Inequality comparisons are primarily for defining ground-water management constraints. For example, a waterquality standard should not be exceeded; discharge to a wetland should be greater than a minimum; or contaminated water from a plume should not cross a specified boundary; these are typical constraints that are added to the objective function as an inequality comparison. Model calibration also is served well by inequality comparisons that incorporate indefinite data in the objective function and constrain parameter estimates. For example, one might not know the water level in a pumped well but it is known that the water level must be higher than the pump intake.

\section{Optimization Parameters}

Optimization parameters are the unknown model input variables that are changed to reduce the discrepancy between simulated and measured observations. Aquifer properties or well-pumping schedules are grouped so that all variables can be modified with a single parameter. Optimization parameters typically are based on common physical, spatial, and temporal relations.

Grouping aquifer properties into optimization parameters constrains the optimization problem and makes the problem more tractable. Objective functions become less sensitive to parameter change and more sensitive to noise as the number of independent parameters increases. In the extreme case of treating all of the aquifer properties and potential pumpage at each node as independent parameters, most optimization procedures cease to function and final parameter estimates are wholly dependent on initial parameter estimates.

Defining optimization parameters typically is more difficult for model calibration than for ground-water management problems. Ground-water flow model calibration typically estimates aquifer properties such as transmissivity, vertical leakance, or recharge and the basis for defining these aquifer properties with zones and parameter-weight matrices tends to be somewhat subjective. Ground-water management problems generally attempt to estimate pumpage schedules. Optimization parameters such as pumping schedules are defined handily because well locations are unambiguous.

Calibration with an excessive number of optimization parameters produces a smaller error between simulated and measured observations. However, corresponding estimates of hydraulic properties are frequently misestimated to a greater degree than those estimated using schemes with fewer parameters. As the number of parameters increases, more parameters are insensitive to model change (Shah and others, 1978; Yeh and Yoon, 1981). Best estimates of hydraulic conductivity and drawdown have been estimated with only a few homogeneous zones in controlled numerical experiments (Freyberg, 1988).

\section{Zonation and Parameter-Weight Matrices}

Zonation and parameter-weight matrices are used in MODOPTIM to define optimization parameters with a common spatial or temporal relation. Zonation assumes the aquifer system can be subdivided into homogeneous zones that are characterized by independent optimization parameters. For example, the hydraulic conductivity distribution in figure 1a was the product of characterizing the distribution with four zones. Abrupt transitions between zones is appropriate when geologic data indicate discontinuous boundaries such as faults or facies changes. Zonation, whether appropriate or not, is used more often than other methods owing to the ease of application (Jahns, 1966; Coats and others, 1970; Veatch and Thomas, 1971; Cooley, 1977; Carrera and Neuman, 1986; and Hill, 1992).

In many instances, the spatial variability of an aquifer property will be conceptualized as a continuous function that smoothly changes from node to node. Zonation cannot adequately describe the expected spatial variability, but a parameter-weight matrix can allow for smooth changes within a distribution and for constant relative change from one node to another. For example, the hydraulic-conductivity distribution in figure $1 \mathrm{~b}$ was the product of characterizing the distribution with

$$
K(x, y)_{n}=a+b\left(w m_{n}\right)
$$



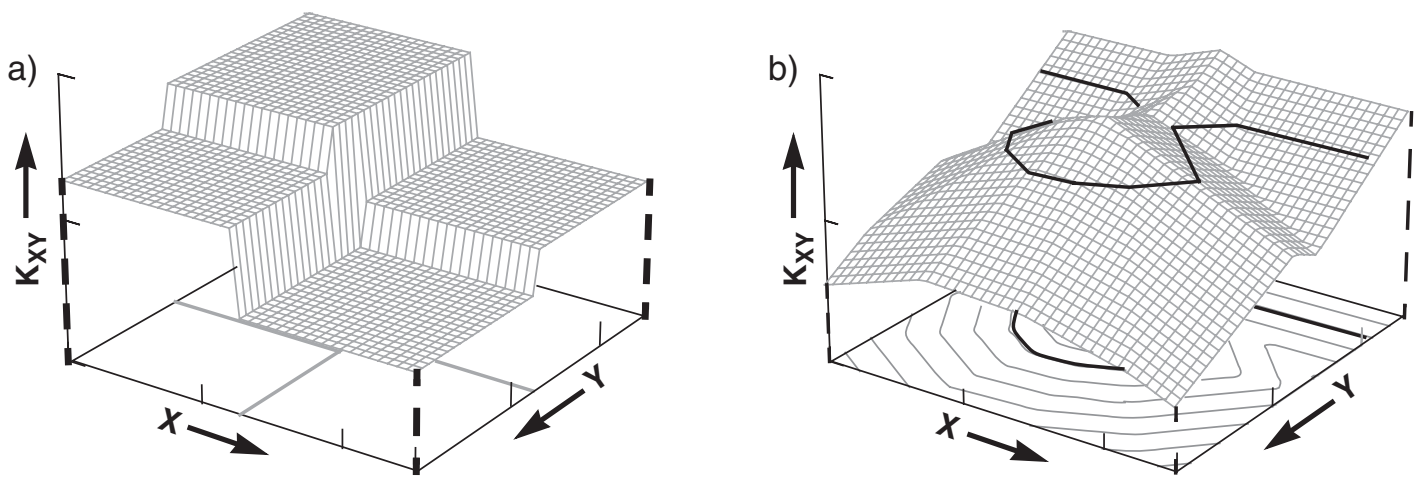

Figure 1. Two hypothetical hydraulic-conductivity distributions: (a) zones and (b) a parameter-weight matrix.

where:

$a$ is a constant,

$b \quad$ is a parameter-weight matrix multiplier, and

$w m_{n} \quad$ is the $n^{\text {th }}$ parameter-weight in matrix WM defining the expected, relative variability of the hydraulic conductivity distribution.

The coefficients $\mathrm{a}$ and $\mathrm{b}$ are the optimization parameters that are estimated. Parameter-weight matrices typically are filled by kriging or some other interpolation scheme that defines the spatial variability of the aquifer properties within the model domain. Subjective information can be used to augment construction of the parameter-weight matrix if the data set that describes the variability of an aquifer property is limited.

Smoothly varying hydraulic properties can be estimated alternatively with gmregularized pilot points (Doherty, 2003). Hydraulic property destitutions are interpolated from pilot points which are arbitrary locations throughout the model domain. The optimization parameters are hydraulic property values at the pilot points. Regularization forces nearby pilot points to have similar values in the absence of observations and creates smoothly varying surfaces. This allows the number of pilot points to exceed the number of observations. Regularized pilot points are a feature of PEST (Model-Independent Parameter Estimation) (Doherty, 2004) and are not supported in MODOPTIM.

\section{Parameter-Weight Matrices from Iso-Value Contours}

Spatial variation of an aquifer property also can be defined with a parameter-weight matrix that is generated from an iso-value contour. A sparse collection of core, log, and production data combined with a depositional model, geologic knowledge, and experience can be used to define the spatial distribution of an aquifer property (Cooley, 2004). An iso-value contour can be inferred from limited data and serve as the basis for computing expected spatial variations of the aquifer property to be estimated. Characterizing an aquifer property with an iso-value contour can constrain the parameter estimates to geohydrologically plausible values if inferences about the distribution of an aquifer property are reasonable.

A parameter-weight matrix is constructed from an iso-value contour by calculating the minimum distance from each model node to the line defined by an appropriate segment of the iso-value contour. A node must fall within the zone of influence of a segment which is defined by the lines that bisect the angles between the two adjacent segments (fig. 2). If a node is in the zone of influence of multiple segments, the minimum node-to-segment distance will identify the appropriate segment and distance. Applying this procedure to all the nodes within a layer or cross section will generate a parameter-weight matrix of distances between nodes and the iso-value contour. 
An aquifer-property distribution, such as hydraulic conductivity, can be generated by using the parameterweight matrix in eq. 4 and estimating the coefficients $a$ and $b$. As an example, two hydraulic-conductivity distributions were produced by multiplying the parameter-weight matrix constructed from figure 2 by two arbitrary values of $b$ $(-0.025$ and +0.025$)$ for $a=50$ (fig. 3). Changes in $a$ displace the entire surface and changes in $b$ affect the slope of the surface. As the slope of the surface increases, the spatial variability of the aquifer property also increases.

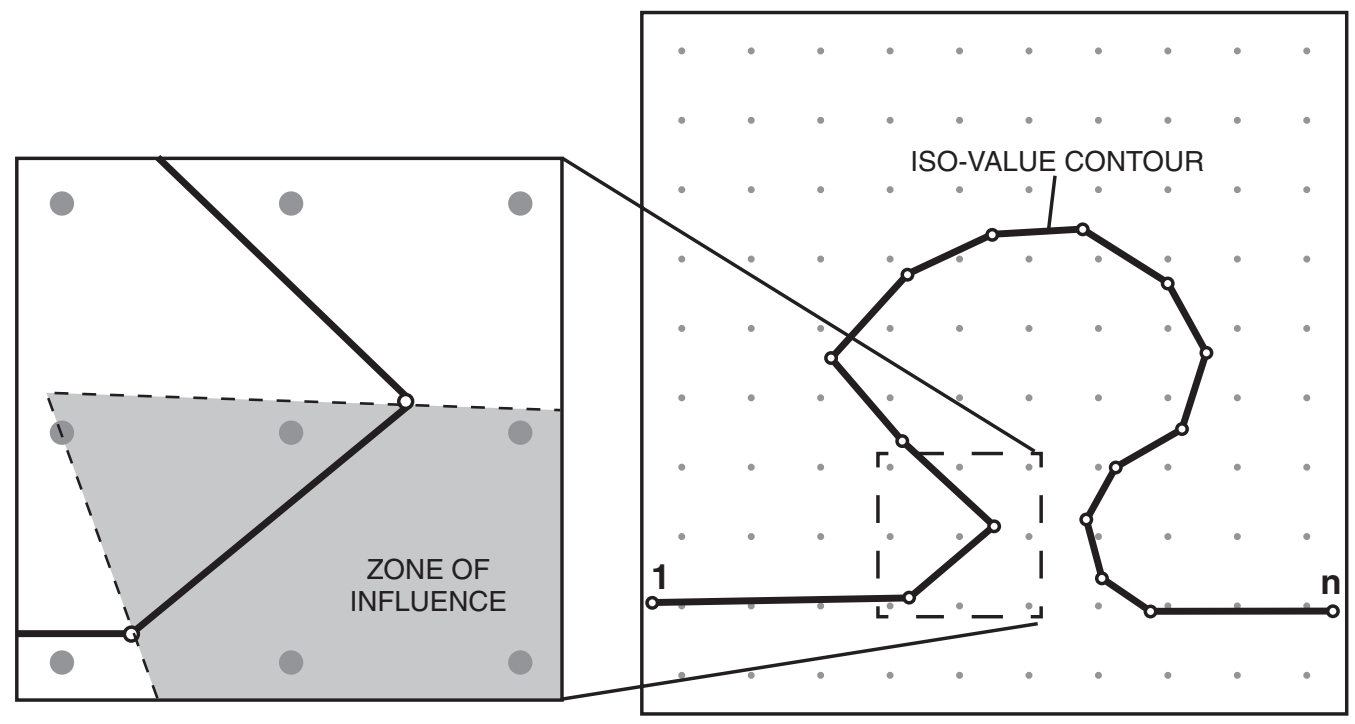

Figure 2. An iso-value contour imposed on a 10-by-10 grid and the zone of influence for a segment of the iso-value contour.

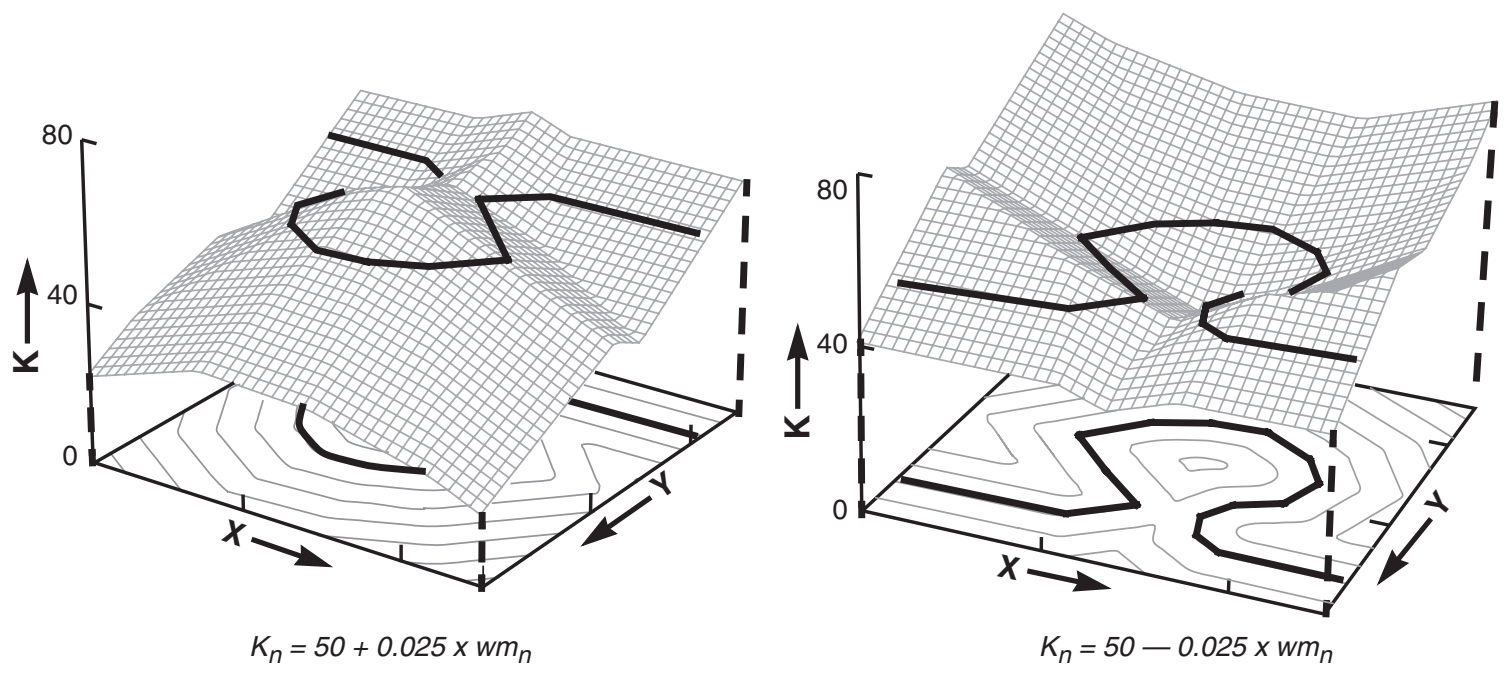

Figure 3. Two hydraulic-conductivity distributions constructed with a parameter-weight matrix of distances to the iso-value contour depicted in figure 2 . 


\section{Optimization Algorithm}

Calibrating ground-water models and optimizing ground-water management scenarios can be iterative processes when the problems are nonlinear. Consequently, optimization algorithms start with an initial set of parameter estimates and solve for a best fit using incremental parameter changes, $p$. The magnitude and direction that each parameter is changed is based on minimization of the objective function, which is the smallest set of differences between the simulated and measured observations.

MODOPTIM uses several gradient-search minimization algorithms that assume the objective function defines a smooth, continuous surface. The performance characteristics of the minimization algorithms are illustrated with a two-parameter example where horizontal hydraulic conductivity, $K_{X Y}$, and vertical hydraulic conductivity, $K_{Z}$ are estimated (Halford and Lovelace, 1994). The error surface of these two parameters was mapped from 0.01 to 1,000 times the calibrated value of $K_{X Y}$ and from 0.01 to 10,000 times the calibrated value of $K_{Z}$ (fig. 4). Parameter changes between iterations and search paths are projected on the error surface.

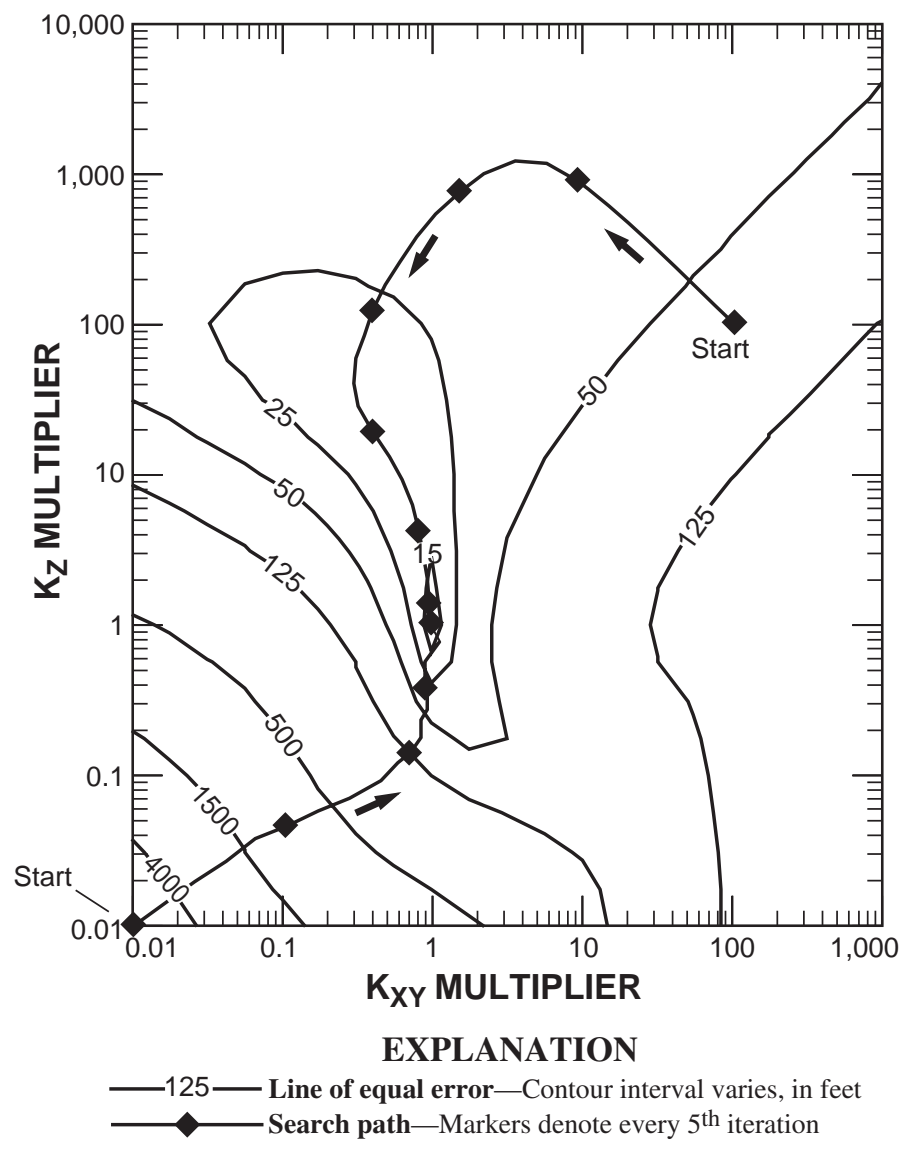

Figure 4. A two-parameter error surface and two steepest-descent search paths.

\section{Minimization Algorithms}

The MODOPTIM algorithm is similar to a steepest-descent solution under limited circumstances. A steepestdescent search direction at optimization iteration $k$ is (Gill and others, 1981)

$$
p_{k}=-g_{k}
$$


where:

npar is the number of parameters being estimated;

$g_{k} \quad$ is the vector of npar gradients at the $k^{\text {th }}$ optimization iteration, $g_{k}=J_{k}{ }^{T}\left(f\left(x_{k}\right)\right)$;

$J_{k}{ }^{T} \quad$ is the transpose of the Jacobian matrix $\left(J_{k}\right)$ at the $k^{\text {th }}$ optimization iteration. The Jacobian or sensitivity matrix of $S S(x)$ is the partial derivative of $S S(x)$ (eq. 1) at all observations with respect to parameter change, is a nobs by npar matrix, and is described by

$$
J=\left[\begin{array}{cccc}
w_{1} \frac{\partial}{\partial \mathrm{x}_{1}}\left(\hat{\mathrm{o}}_{1}\right) & w_{1} \frac{\partial}{\partial x_{2}}\left(\hat{o}_{1}\right) & \ldots & w_{1} \frac{\partial}{\partial x_{\text {npar }}}\left(\hat{o}_{1}\right) \\
w_{2} \frac{\partial}{\partial \mathrm{x}_{1}}\left(\hat{\mathrm{o}}_{2}\right) & w_{2} \frac{\partial}{\partial x_{2}}\left(\hat{o}_{2}\right) & \ldots & w_{2} \frac{\partial}{\partial x_{\text {npar }}}\left(\hat{o}_{2}\right) \\
\vdots & \vdots & \vdots \\
w_{n o b s} \frac{\partial}{\partial \mathrm{x}_{1}}\left(\hat{\mathrm{o}}_{n o b s}\right) w_{n o b s \frac{\partial}{\partial x_{2}}\left(\hat{o}_{n o b s}\right)} & \ldots . w_{n o b s} \frac{\partial}{\partial x_{n p a r}}\left(\hat{o}_{n o b s}\right)
\end{array}\right] \downarrow
$$

$f\left(x_{k}\right) \quad$ is the vector of nobs residuals (eq. 2) at the $k^{\text {th }}$ optimization iteration, and

$x_{k} \quad$ is the vector of npar parameter estimates at the $k^{\text {th }}$ optimization iteration. The parameter estimates are updated to the next iteration by

$$
x_{k+1}=x_{k}+p_{k} .
$$

Step length is fixed a priori or scaled relative to the error change at the previous step. If the derivative surface is smooth, the path travelled along the error surface (fig. 4) is similar to that of a marble rolling down a hillside.

Steepest-descent algorithms have a guaranteed convergence on a minimum, but the rate of convergence is generally slow (Gill and others, 1981). The penalty imposed by this slow rate of convergence is great enough to warrant the use of other methods.

The predominant minimization algorithms in MODOPTIM are variations of Newton's method. Model improvement is still iterative, but the search direction is solved for with first and second derivatives of a quadratic objective function, SS(x) (Gill and others, 1981). The first three terms of a Taylor-series expansion about the historymatching parameters at iteration $\mathrm{k}$ form a quadratic model of the objective function in terms of $p_{k}$ :

$$
S S\left(x_{k}+p_{k}\right)=S S_{k}\left(x_{k}\right)+g_{k}^{T} p_{k}+\frac{1}{2} p_{k}^{T} G_{k} p_{k} .
$$

The minimization of $S S\left(x_{k}+p_{k}\right)$ with respect to $p_{k}$ defines the best search direction. The first derivative of $S S\left(x_{k}+p_{k}\right)$ when set to 0 gives the appropriate search direction

$$
G_{k} p_{k}=-g_{k}
$$

where:

$G_{k} \quad$ is the Hessian matrix of $S S(x)$ at the $k^{t h}$ optimization iteration or $G_{k}=\left(J_{k}^{T} J_{k}+Q_{k}\right)$, and

$Q_{k} \quad$ is the sum of residuals times the second partial derivatives of $f\left(x_{k}\right)$. 
Newton's method itself is not used in MODOPTIM because explicit evaluation of the second-order terms is too computationally intensive. The first-order term $J_{k}^{T} J_{k}$ of the Hessian matrix $G_{k}$ is evaluated with npar simulations and explicit evaluation of the second-order term $Q_{k}$ requires $n p a r$ by npar simulations. The high cost of evaluating $Q_{k}$ is avoided by either neglecting or approximating $Q_{k}$.

The Gauss-Newton method is used in MODOPTIM and reduces the cost of evaluating $G_{k}$ by assuming that the second-derivative term $Q_{k}$ can be neglected because $\left\|f\left(x_{k}\right)\right\|$ residuals and sum-of-squares error tend to zero near the minimum. As a consequence, $Q_{k}$ also will tend to zero. The Gauss-Newton search direction is

$$
\left(J_{k}^{T} J_{k}\right) p_{k}=-g_{k}
$$

A comparison between a Gauss-Newton path and a steepest-descent path shows that the final parameter estimates were obtained with considerably fewer iterations by the Gauss-Newton solution (figs. 5 and 6). The initial search direction of the Gauss-Newton solution differed from the steepest-descent direction because the Hessian matrix $\left(J_{k}^{T} J_{k}\right)$ includes the information that the vertical hydraulic-conductivity gradient is decreasing and the horizontal hydraulic-conductivity gradient is increasing (fig. 5). Although the Gauss-Newton algorithm converged on the minimum in a relatively few iterations, the search path did include a segment from iteration 2 to 3 where RMS error increased (fig. 6), a result of overestimating the step length and overshooting the minimum. New parameter estimates tend to overshoot the minimum where the rate of gradient increase is underestimated (fig. 7). Likewise, new parameter estimates will undershoot the minimum where the rate of gradient decrease is underestimated.

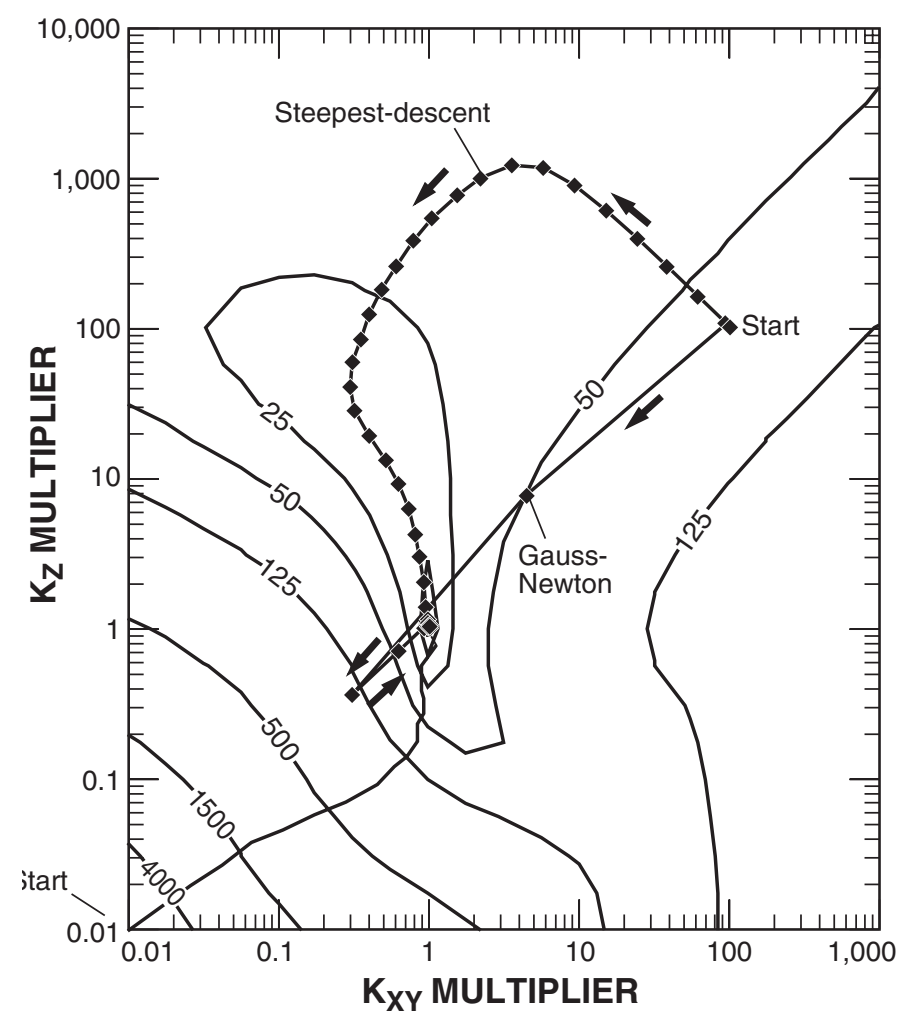

EXPLANATION

$-125-$ Line of equal error-Contour interval varies, in feet
Search path-Markers denote each iteration

Figure 5. A steepest-descent and a Gauss-Newton search path.

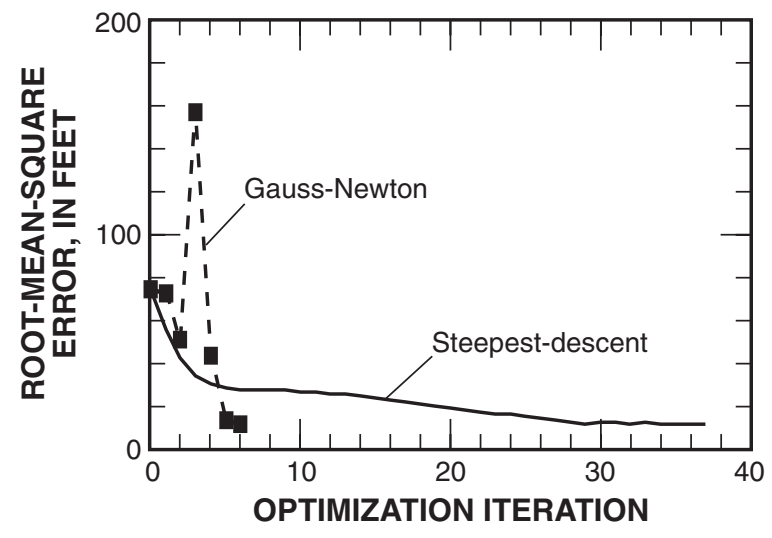

Figure 6. The root-mean-square error change of the search paths shown in figure 5 . 


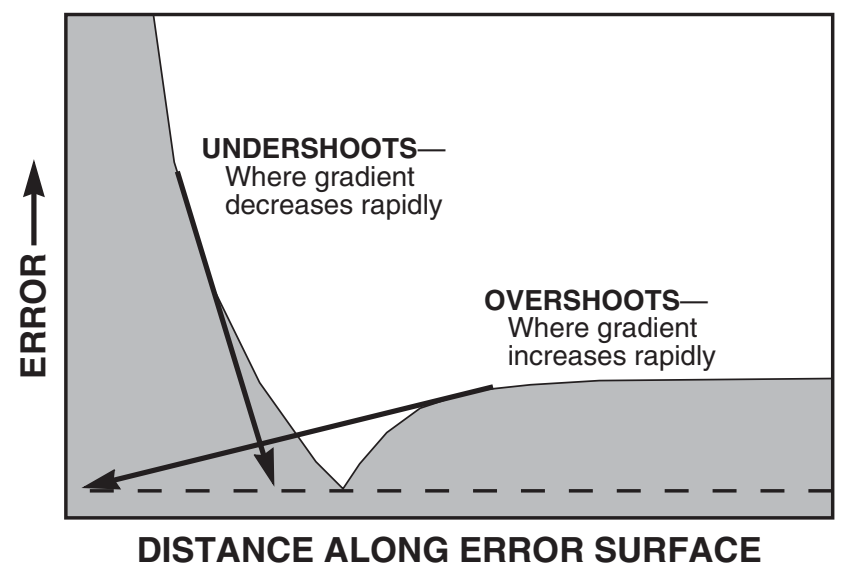

Figure 7. Errors in search direction and range from the Gauss-Newton approximation.

The Levenberg-Marquardt solution (Marquardt, 1963) is a compromise between Gauss-Newton and a steepestdescent solution. The rate of convergence on the minimum by the Levenberg-Marquardt solution is not as rapid as the Gauss-Newton solution, but the Levenberg-Marquardt solution is more robust. The Levenberg-Marquardt solution can constrain parameter changes such that the RMS error always decreases. This can stabilize highly non-linear problems and is comforting where the user does not have the benefit of an error surface. The Levenberg-Marquardt search direction is

$$
\left(J_{k}^{T} J_{k}+\lambda_{k} I\right) p_{k}=-g_{k}
$$

where:

$\lambda_{k} \quad$ is an arbitrary, positive scalar value, and

$I \quad$ is the identity matrix.

The term $\lambda_{k}$ is defined relative to Euclidean norm of $J_{k}\left\|J_{k}\right\|$. For $\lambda_{k}$ much greater than $\left\|J_{k}\right\|$, the step length becomes small and the search direction becomes a steepest-descent direction. As $\lambda_{k}$ approaches 0 , the term $\lambda_{k} \mathrm{I}$ also goes to 0 and the search direction is a Gauss-Newton direction. The term $\lambda$ has been defined in several ways by different authors (Marquardt, 1963; Cooley, 1977; Watson and Lee, 1986; Hill, 1992) and is defined in MODOPTIM as

$$
\lambda_{k}=\alpha \times \max \left\{J_{k}^{T} J_{k}\right\}
$$

where $\alpha$ is a user-defined constant.

The effect of $\alpha$ on the search direction greatly depends on the current parameter estimates. The selection of $\alpha$ will not affect search direction if parameter estimates are in a region of decreasing gradient (fig. 8). Varying $\alpha$ from 0 to 1 in areas of increasing gradient can produce vectors of parameter change that are nearly orthogonal to one another and differ in length by an order of magnitude. Step length decreases as $\alpha$ increases because $\lambda_{k}$ also increases, regardless of the current parameter estimates. The value of $\alpha$ should not exceed 1 because the optimization algorithm will converge slowly (fig. 8). 


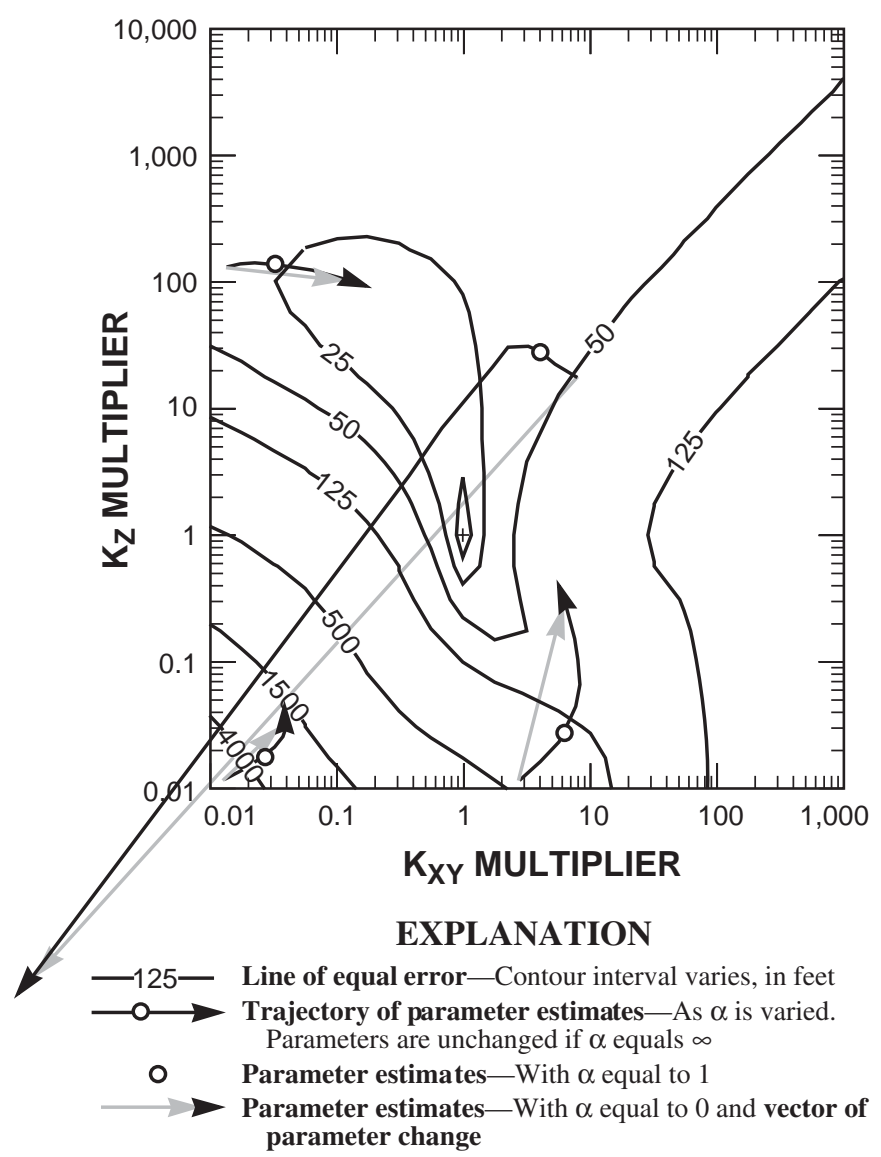

Figure 8. Effect of the variable a on the direction and magnitude of parameter change.

Quasi-Newton methods attempt a closer approximation of $G_{k}$ by approximating $Q_{k}$ instead of negating it. This is useful when the combined measurement and model errors do not tend to zero. Quasi-Newton methods maintain an approximation of $Q_{k}$ by comparing first-order changes between iterations. Many different schemes, which are sometimes referred to as variable-metric methods, have been devised to update $M_{k}$ which is the approximation of $Q_{k}$ (Gill and others, 1981). The Broyden-Fletcher-Goldfarb-Shanno (BFGS) update (Gill and others, 1981) is an effective $M_{k}$ update algorithm and is used in MODOPTIM. The quasi-Newton direction is

$$
\left(J_{k}^{T} J_{k}+M_{k}\right) p_{k}=-g_{k}
$$

and the BFGS update is

$$
M_{k}=M_{k-1}-\frac{W_{k} s_{k} s_{k}^{T} W_{k}}{s_{k}^{T} W_{k} s_{k}}+\frac{y_{k} y_{k}^{T}}{y_{k}^{T} s_{k}}
$$

where:

$$
W_{k}=J_{k}^{T} J_{k}+M_{k-1}
$$




$$
\begin{gathered}
s_{k}=x_{k}-x_{k-1} \text {, if no step-length criteria are exceeded, } s_{k}=p_{k} \\
y_{k}=g_{k}-g_{k-1} .
\end{gathered}
$$

Parameters have been estimated with quasi-Newton algorithms in ground-water models (Carrera and Neuman, 1986; Hill, 1992; and Poeter and Hill, 1998) and petroleum reservoir models (Yang and others, 1987; and Halford, 1992).

Quasi-Newton algorithms periodically revert to either a Gauss-Newton or Levenberg-Marquardt minimization algorithm because $M_{k}$ cannot be estimated or the approximation of $Q_{k}$ has failed for a given iteration. The matrix $M_{k}$ cannot be estimated for the first iteration because prior parameter and gradient estimates do not exist. After several iterations, the update of the matrix $M_{k}$ will fail because the change of gradients with respect to the parameter changes becomes a poor approximation of the second-order information in $Q_{k}$. The update of the matrix $M_{k}$ typically fails after a relatively large parameter change.

\section{Parameter Sensitivity and Ranking of Parameters}

Parameter sensitivity describes how parameter changes affect model error and typically is depicted as a graph of error change versus parameter change (fig. 9). More sensitive parameters have steeper slopes than less sensitive parameters. In this example, model error is more sensitive to changes in natural recharge $\left(x_{1}\right)$ than to changes in the vertical anisotropy $\left(x_{2}\right)$ or the underflow recharge rate $\left(x_{3}\right)$.

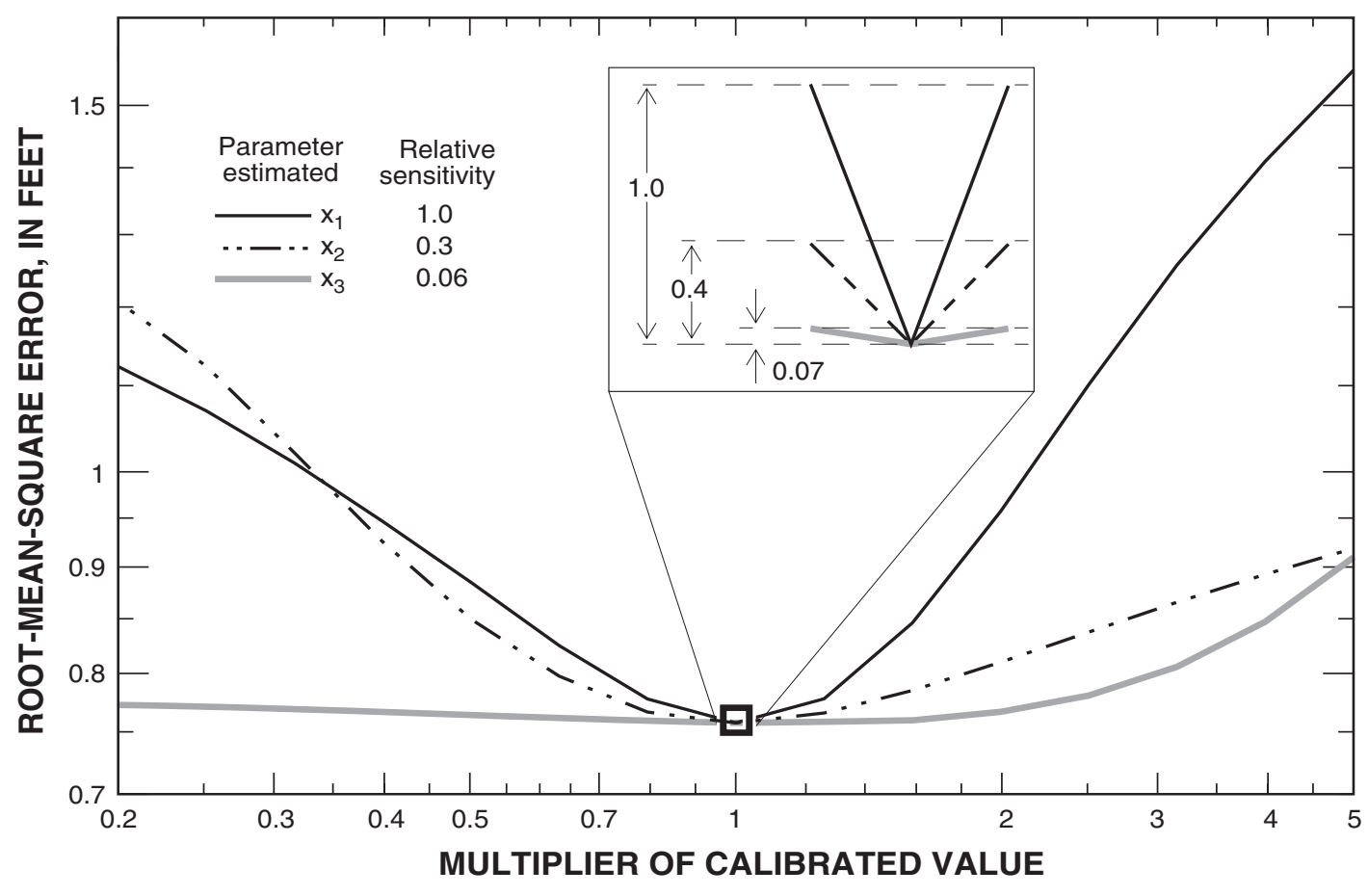

Figure 9. Comparison of the relative sensitivity of natural recharge $(x 1)$, vertical anisotropy $(x 2)$, and underflow recharge $(x 3)$ to root-mean-square error change as these same parameters are varied independently. 
Parameter sensitivity can be used as a measure of the "reasonableness" of estimating a parameter and as a guide for which parameters should be estimated. Conceptually, one should not be attempting to estimate insensitive parameters because there is no basis to do so as measured by the objective function. Pragmatically, insensitive parameters should not be estimated with MODOPTIM because the quasi-Newton minimization algorithm becomes ineffectual. Ineffectual solutions are characterized by large changes of insensitive parameters and negligible reduction of the objective function.

Parameter sensitivity is quantified in MODOPTIM by the main diagonal of the $J^{T} J$ matrix, which is similar to composite-scaled sensitivities as defined by other investigators (Poeter and Hill, 1997). The sensitivity of the $j^{t h}$ parameter is

where:

$$
\left(J^{T} J\right)_{j, j}=\sum_{i=1}^{n o b s}\left(w_{i} \frac{\partial}{\partial x_{j}}\left(\hat{o}_{i}\right)\right)^{2}
$$

$w_{i} \frac{\partial}{\partial x_{j}}\left(\hat{o}_{i}\right) \quad \begin{aligned} & \text { is the weighted-sensitivity coefficient of the } i^{t h} \text { observation with respect to the } j^{t h} \text { parameter } \\ & \text { estimated. }\end{aligned}$

The measure calculated by eq. 18 increases as parameter sensitivity increases for a given model and objective function. Estimated parameters in MODOPTIM are ranked from most sensitive to least sensitive (eq. 18).

Parameter sensitivity is reported by MODOPTIM in terms of relative sensitivity which is

$$
R S_{j}=\sqrt{\frac{\left(J^{T} J\right)_{j, j}}{\max \left[\left(J^{T} J\right)_{j, j}\right]}}
$$

for the $j^{\text {th }}$ parameter (fig. 9). The most sensitive parameter will have a relative sensitivity $\left(R S_{j}\right)$ of 1 and the relative sensitivity of all the other estimated parameters will be less than 1 . The threshold for identifying insensitive parameters and not estimating those parameters is defined with a relative scale (eq. 19) because large, relative differences between parameter sensitivities render the optimization algorithm ineffectual. The square-root is taken in equation 19 to make the relative sensitivities similar in scale to the more familiar plots of RMS error change versus parameter change (fig. 9). Parameters estimated should have a relative sensitivity of $\geq 0.01$ (Hill, 1998).

\section{Parameter Scaling}

Estimating multipliers of the parameters instead of the parameters themselves is a more useful form of parameter scaling. However, estimation of multipliers does not entirely remedy problems associated with estimating log-normally distributed parameters, such as hydraulic conductivity. Estimating log-parameters makes incremental parameter adjustments more appropriate for the minimization algorithm, in addition to reducing differences between the magnitudes of the values estimated. Estimation of the log-parameters transforms parameter change from a multiplicative relation to an additive one. This is more appropriate because the incremental parameter change estimated by the minimization algorithm is additive (eq. 7). Log-parameters also constrain estimates to positive values, which is a reasonable constraint for parameters such as hydraulic conductivity.

Parameters can be scaled over a known range if reasonable lower and upper limits can be assigned. Parameters are scaled in MODOPTIM that vary from 0 to 1 by

where:

$$
x_{\text {Scaled }}=\frac{x-x_{L}}{x_{U}^{-x_{L}}}
$$

$x_{U} \quad$ is the upper limit of parameter $x$, and

$x_{L} \quad$ is the lower limit of parameter $x$. 
Scaling parameters with eq. 20 allows the parameters to change sign while adjusting parameter sensitivity.

Scaling parameters affects parameter sensitivity and the associated error surfaces as illustrated by a twoparameter example (fig. 10). The error surface associated with $K_{X Y}$ and $K_{Z}$ unscaled (fig. 10a) shows the model is more sensitive to estimates of $K_{X Y}$ or $K_{Z}$ that are less than the calibrated estimates of $(63,10)$. Parameter sensitivity is more uniform across the error surface and is symmetric about the calibrated values after log-transforming $K_{X Y}$ and $K Z$ (fig. 10b). Parameter sensitivity of $\log 10\left(K_{X Y}\right)$ and $\log 10\left(K_{Z}\right)$ are constants across the entire error surface, but the model is still twice as sensitive to changes in $\log 10\left(K_{X Y}\right)$ than $\log 10\left(K_{Z}\right)$. The sensitivity of $\log \left(K_{X Y}\right)$ is made similar to $\log \left(K_{Z}\right)$ by applying eq. 20 and scaling $\log \left(K_{Z}\right)$ from 0 to 2 and scaling $\log 10\left(K_{X Y}\right)$ from 1.4 to 2.2 (fig. 10c). A symmetrical error surface makes optimization more stable and less sensitive to initial parameter estimates.

\section{Implementation of Minimization Algorithm in MODOPTIM}

MODOPTIM is initialized by executing MODFLOW once to establish initial differences between simulated and measured observations. These differences, or residuals, are then minimized by MODOPTIM to decrease the sumof-squares objective function. The weighted-sensitivity coefficients of the Jacobian matrix are approximated by the influence coefficient method (Yeh, 1986). Approximate sensitivities function as well as exact sensitivities (Yager, 2003) but are easier to compute and numerically more robust. Weighted-sensitivity coefficients are the derivatives of observation change with respect to parameter change. Each weighted-sensitivity coefficient is approximated by

where:

$$
w_{i} \frac{\partial}{\partial x_{j}} \hat{o}_{i}(x) \frac{w_{i}\left(\hat{o}_{i}\left(x_{j}+\Delta x_{j}\right)-\hat{o}_{i}\left(x_{j}\right)\right)}{\Delta x_{j}}
$$

$$
\begin{array}{ll}
w_{i} & \text { is the } i^{t h} \text { observation weight, } \\
\hat{o_{i}}\left(x_{j}+\Delta x_{j}\right) & \text { is the } i^{t h} \text { observation simulated with the } j^{\text {th }} \text { parameter perturbed, } \\
\hat{o_{i}}\left(x_{j}\right) & \text { is the } i^{\text {th }} \text { observation simulated in the reference simulation, and } \\
\Delta x_{j} & \text { is the incremental perturbation of the } j^{\text {th }} \text { parameter. }
\end{array}
$$

Each parameter is changed a small amount $\left(\Delta x_{j}\right)$ and MODFLOW is used to compute new water levels and flow rates for each perturbed parameter. For each optimization iteration, MODFLOW is called npar +1 times. The first-order $\left(J_{k}^{T} J_{k}\right.$; eq. 6) and second-order ( $M_{k}$; eq. 14$)$ terms of the Hessian matrix and the vector of gradients $\left(g_{k}\right.$; eq. 5$)$ are calculated after the Jacobian matrix has been constructed. The matrices $J_{k}^{T} J_{k}, M_{k}$, and $g_{k}$ are referred to in the MODOPTIM output files as $F I R_{k}, S E C D_{k}$, and $G R 1_{k}$, respectively.

Estimated parameters and all associated matrices are ranked from most sensitive to least sensitive (eq. 19) which is defined by the main diagonal of $F I R_{k}$. For example,

$$
F I R_{k}=\left[\begin{array}{lll}
1 & 1 & 0 \\
1 & 5 & 3 \\
0 & 3 & 2
\end{array}\right], G R 1_{k}=\left[\begin{array}{l}
3 \\
1 \\
2
\end{array}\right] \quad \underset{\text { RANKING }}{\mathrm{AFTER}} \quad F I R_{k}=\left[\begin{array}{lll}
5 & 3 & 1 \\
3 & 2 & 0 \\
1 & 0 & 1
\end{array}\right], G R 1_{k}=\left[\begin{array}{l}
1 \\
2 \\
3
\end{array}\right]
$$

The parameter in the first row after ranking has the greatest relative sensitivity which is equal to 1 . The number of parameters estimated will be reduced if any of the relative sensitivities fall below a user-defined threshold. These insensitive parameters are held at the estimated value from the previous iteration. In the preceding example, the relative sensitivities of the three parameters are $1.00,0.63$, and 0.45 , respectively. If the relative sensitivity threshold is equal to 0.5 , two parameters would be estimated instead of three. 
The parameter change vector $\left(p_{k}\right)$ is solved with a quasi-Newton algorithm (eq. 13) as long as the matrix of second-order information $\left(M_{k}\right)$ remains positive definite. If the update of $M_{k}$ fails, prior parameter change information does not exist, or the solution of equation 13 results in an error increase, then the parameter change vector is solved with a Levenberg-Marquardt algorithm (eq. 11). If the length of the parameter change vector exceeds a user-defined limit, the vector $\left(p_{k}\right)$ is scaled to that limit. Parameters that become constrained by limits can be permanently eliminated from the matrix of actively estimated parameters (fig. 11).

Optimization continues iteratively until a user-defined convergence has been reached or maximum number of iterations has been exceeded. Convergence is achieved when error reduction is small or parameter change is slight. Model error is updated to reflect the latest parameter estimates and a new iteration begins if convergence has not been achieved.

a)

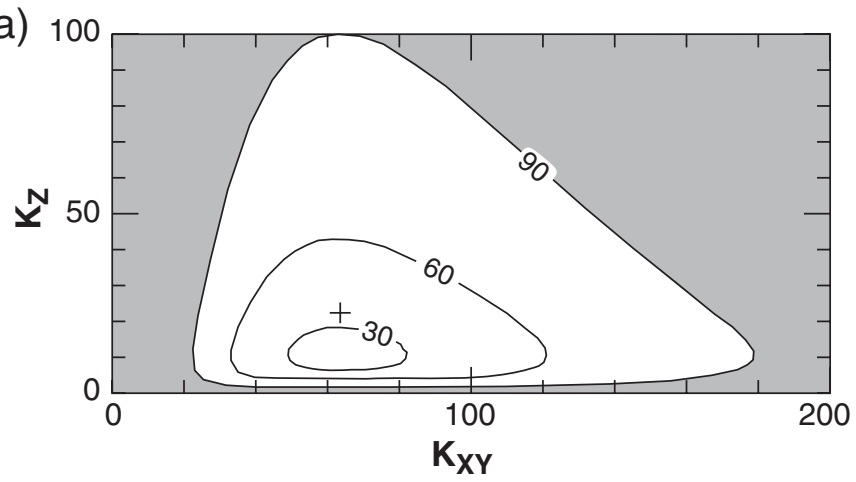

Figure 10. Effect of log transforms and scaling on parameter sensitivity.

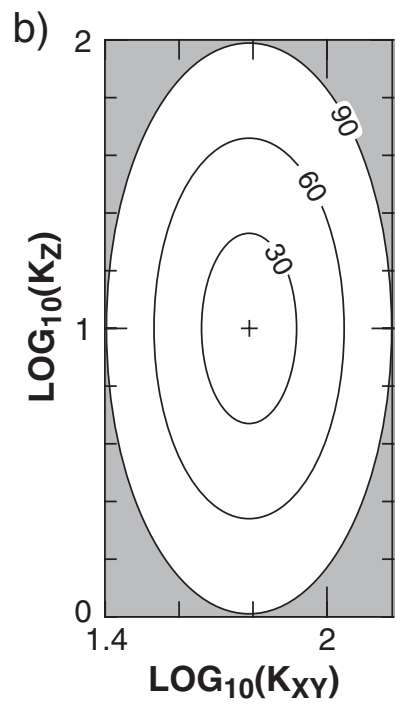

c)
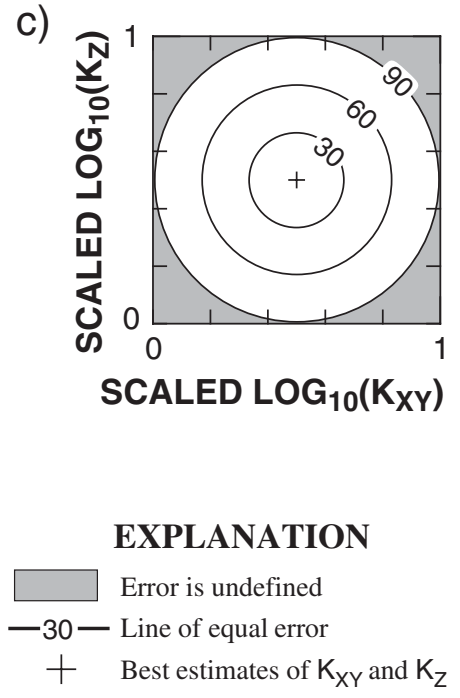

\section{MODOPTIM—ORGANIZATION AND STRUCTURE}

MODOPTIM has been implemented as a ground-water model calibration and management tool that uses MODFLOW-96 (McDonald and Harbaugh, 1988; Harbaugh and McDonald, 1996) as a subroutine. Modifications to MODFLOW are minimal to facilitate the addition of new modules and future upgrades of MODFLOW. Parameter estimates directly modify hydraulic properties and stresses in standard MODFLOW input files. Simulated water levels, flow rates, water-quality values, and pumping-lift costs are extracted from MODFLOW by calls to MODOPTIM subroutines added to the main routine of MODFLOW.

\section{MODOPTIM and MODFLOW files}

Information needed by MODOPTIM is read from three primary files that define the optimization problem, the MODFLOW model, and the coordinate system. The primary files, master optimization, combined

MODOPTIM/MODFLOW input, and three-dimensional model grid files will be referred to by the suggested suffixes OPT, INP, and GR3, respectively. This naming convention is not required by MODOPTIM but is a method of identifying input files. Additional files with output controls, solvers, and known stresses are needed by MODFLOW, but information in files other than the three primary files is not used directly by the optimization routine. 


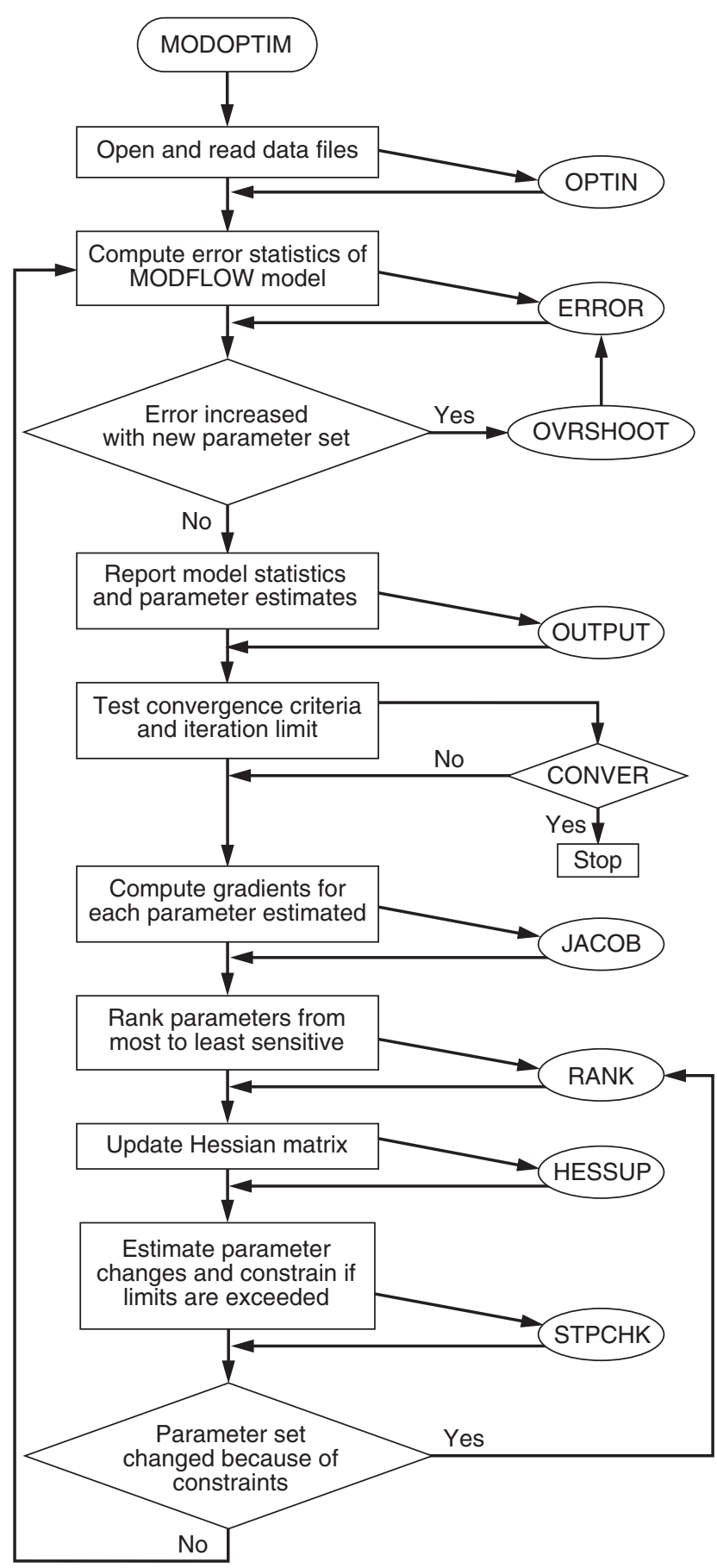

The optimization problem is defined in the OPT file which the user identifies at the command prompt after MODOPTIM has been executed. The OPT file contains information on other files to be opened, parameter-weight matrices, optimization parameters, and observations. Information in the OPT file is used exclusively by the optimization routine. Input instructions and definitions of variables specified in the OPT file are detailed in Appendixes A and B.

The combined MODOPTIM/MODFLOW input file (INP) contains information to construct the BAS file and MODFLOW files with hydraulic properties and stresses to be estimated. The BAS file is constructed from the combined input file so that model dimensions, number of stress periods, and aquifer extent (IBOUND arrays) are entered only once. Information in the constructed BAS file is read each time the MODFLOW subroutine is called and is not passed directly from MODOPTIM. Other MODFLOW files that are constructed from the INP file are BCF, WEL, MNW, RCH, ET, GHB, HFB, DRN, RIV, STR, $\mathrm{CHD}$, and VAR files. Input instructions and definitions of variables specified in the INP file are detailed in Appendix C.

MODFLOW files are constructed from the composite MODFLOW/MODOPTIM file to facilitate placement of name tags (variable pname) that identify hydraulic characteristics and stresses to be modified. Hydraulic characteristics and stresses in MODFLOW that can be estimated by MODOPTIM are read from files as ncol by nrow arrays (storage, transmissivity, vertical leakance, recharge, evapotranspiration) or as cell-by-cell lists (general head boundaries, rivers, specified heads, drains, wells).

The dimensions of the columns, rows, and layers and widths and thicknesses of the model cells are specified in the GR3 file. Column and row offsets and node elevations also are contained in the GR3 file to relate model coordinates to digitized features and well locations. Input instructions and definitions of variables specified in the GR3 file are detailed in Appendix D.

Vertical anistropy can be defined and estimated with the Time-Variant Hydraulic-Property (VAR1) Package (Halford, 1998). The VAR1 package was developed originally to accomodate temporal changes in inter-node conductance, hydraulic conductivity, the top and bottom elevations of model layers, and the primary and secondary storage coefficients. Modifying

Figure 11. Flow chart of MODOPTIM main program 
all conductances along either columns or rows with the VAR1 package allows vertical anistropy to be defined as a multiplier. Input instructions and definitions of variables specified in the VAR1 package are detailed in Appendix E.

\section{Coordinate Systems}

Well locations, aquifer extents, hydrologically distinct zones, drainage basins, and other spatially distributed features typically are mapped in Cartesian coordinates (x, y, z) (fig. 12). These same spatially distributed features are discretized and located by layer (k), row (j), and column (i) indices within MODFLOW (fig. 12). Observation locations, zones, and parameter-weight matrices can be defined in MODOPTIM with either coordinate system or a mixture of the two coordinate systems. For example, the mapped location of an observation well could be defined with Cartesian coordinates $(\mathrm{x}, \mathrm{y})$ and screened interval could be defined by the layer index $(\mathrm{k})$.

Cartesian coordinates and model indices are related to one another by a reference node (fig. 12) where a Cartesian coordinate $(x, y)$ is specified to be equivalent to a node at indices $(i, j)$. If a reference node is not specified, the lower, left corner of the model grid is assumed to be the Cartesian coordinate $(0,0)$. Vertical positions of model nodes are specified directly in a three-dimensional matrix of ncol by nrow by nlay elevations because MODFLOW allows for a vertically deformed model grid. Consequently, the elevation of a simulated observation or hydrologic feature is a function of the column and row indices, in addition to the layer index.

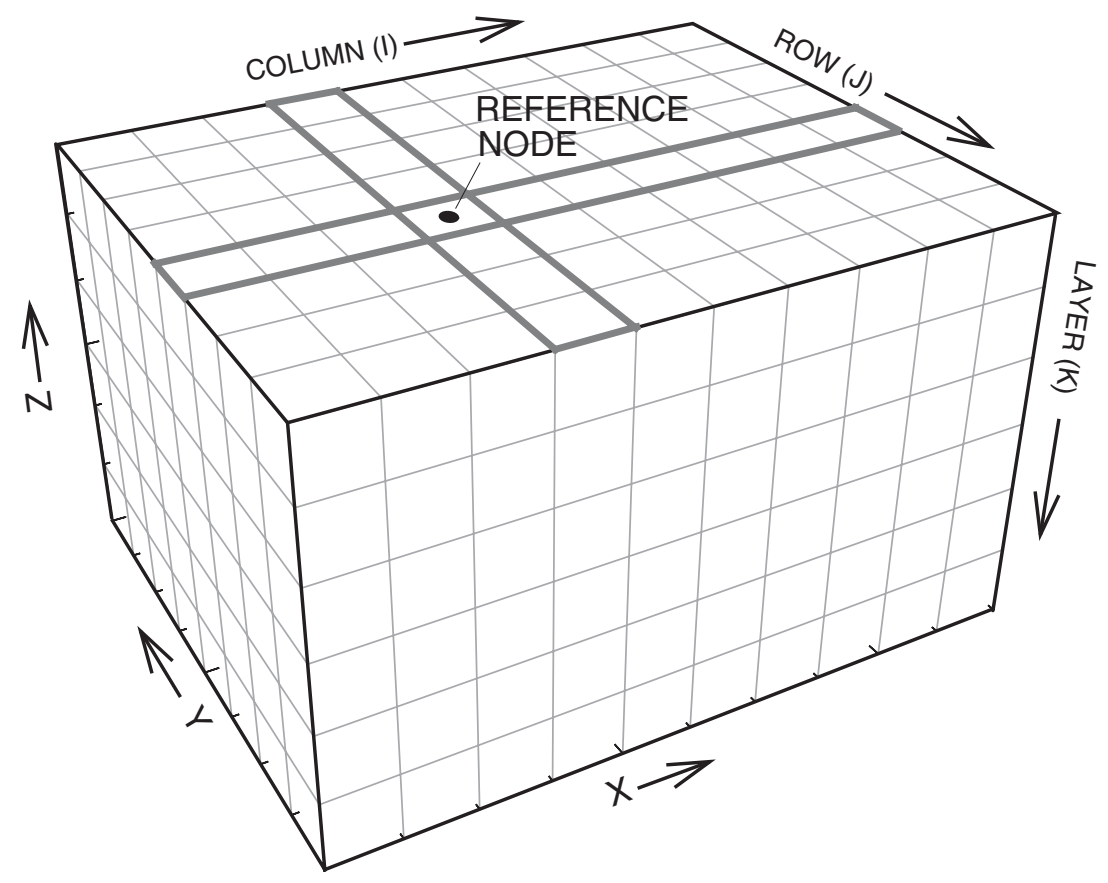

Figure 12. Relation between Cartesian coordinate system and layer, row, and column indices that are used to locate observation points, define zones, and construct weight matrices from iso-value lines. 


\section{Zonation}

A model domain is divided into zones so optimization parameters can modify subvolumes of a hydraulic characteristic or a stress. Subvolumes typically represent geologically distinct zones and areas with different land covers. Boundaries between zones might correspond with geologic discontinuities such as faults, facies changes, and outcrop areas or changes in land use such as the transition from urban to agricultural land use.

Subvolumes within an aquifer system are defined by zones in MODOPTIM. Each zone is identified by a unique integer value that is mapped in the threedimensional integer array, IZONE. The IZONE array can be specified directly or with polygons (Appendix C). Polygons are translated to discretized zones by evaluating whether model nodes lie inside or outside a defined polygon (fig. 13). Zones typically are defined by all nodes that lie within a polygon but can be defined as all nodes that lie outside of a polygon.

Zones are elemental building blocks that are grouped together and define subvolumes modified by optimization parameters. A subvolume can be an individual zone or an aggregate of many zones. For example, the model depicted in figure 13 is subdivided into zones 1, 4, and 7, which are the undifferentiated, discharge, and outcrop areas, respectively. Zone 0 denotes inactive model cells. Recharge could be estimated with two optimization parameters: one for the outcrop area (zone 7) and another for the undifferentiated and discharge areas (zones 1 and 4).

Stress locations to be estimated in ground-water management problems typically are well defined and confined to individual nodes. Consequently, single-cell zones are helpful for defining optimization parameters that modify the flow rates from individual wells. Zones can be defined by individual cells in any of the list directed MODFLOW input files (CHD, DRN, GHB, MNW, RIV, STR, VAR, or WEL packages) that are constructed by MODOPTIM.
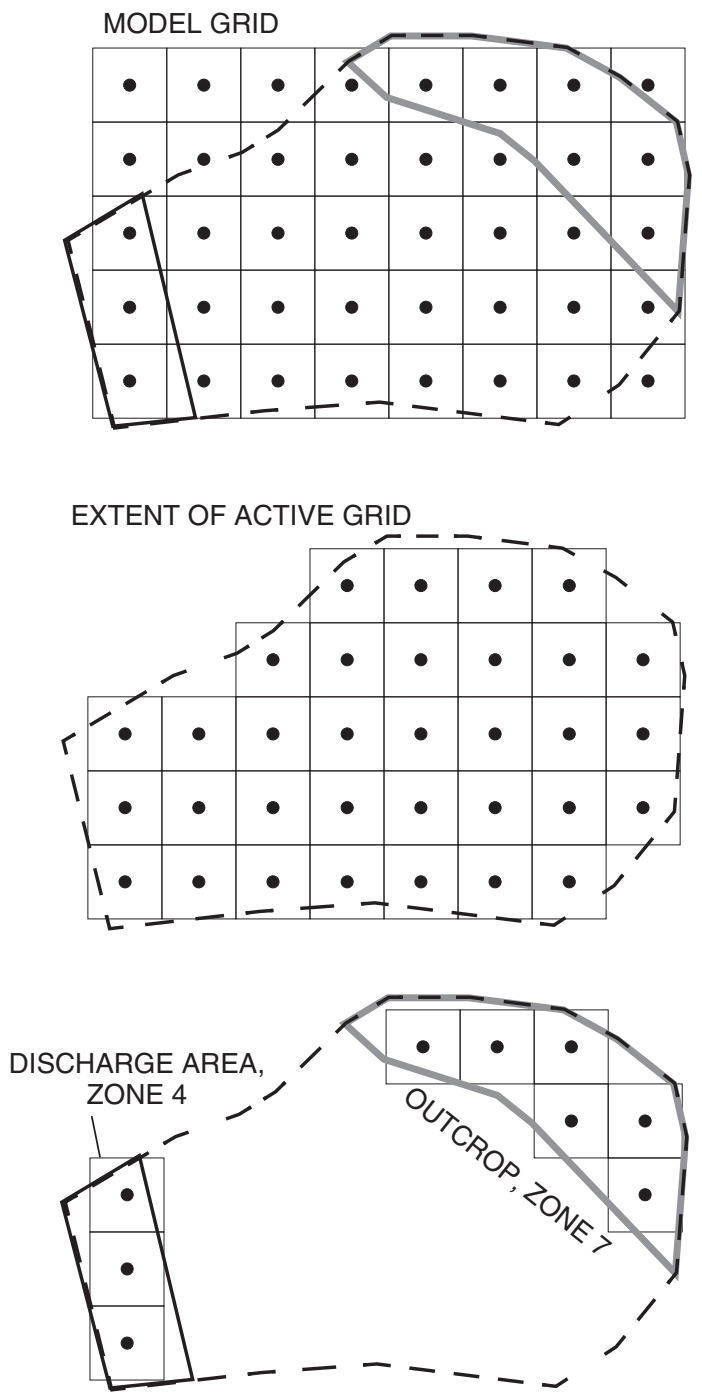

IZONE ARRAY

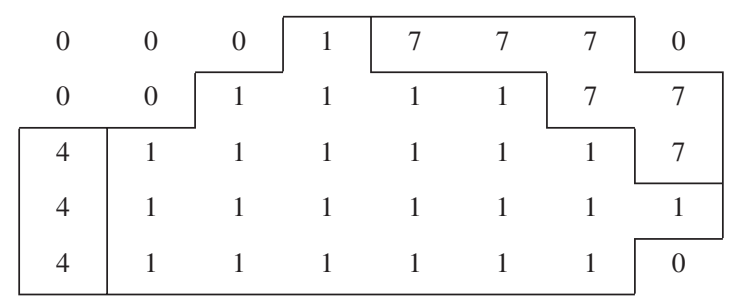

Figure 13. Example of an IZONE array constructed from three polygons. 
Zones also define subvolumes of spatially distributed flow-rate, water-quality, and pumping-lift cost observations, which will be referred to as flow zones. Flow zones that define the areal extent of these observations are stored in the IOBFLX array. Flow zones (IOBFLX) are initially set equal to parameter zones (IZCUBE) and remain so unless a separate IOBFLX array is specified. A separate set of flow zones was allocated and defined because parameter zones that define geologic features do not necessarily coincide with flow zones that approximate drainage basins. Zones can proliferate and become unwieldy if parameter and flow zonation are defined with a single array. For example, a model with 10 parameter zones and 8 drainage basins might need as many as 80 unique zones if only one zone array was used.

\section{Parameter-Weight Matrices}

An assumed variation of hydraulic properties and stresses can be defined with parameter-weight matrices in MODOPTIM (fig. 14). Parameter-weight matrices describe expected spatial variability of hydraulic properties and stresses and expected temporal variability of a stress such as a pumping schedule. Parameter-weight matrices can be one dimensional (nrow, ncol, or nlay), two dimensional (nrow by ncol or ncol by nlay or nrow by nlay), three dimensional (nrow by ncol by nlay), or temporal (iper). Input instructions for defining parameter-weight matrices are detailed in Appendix B.
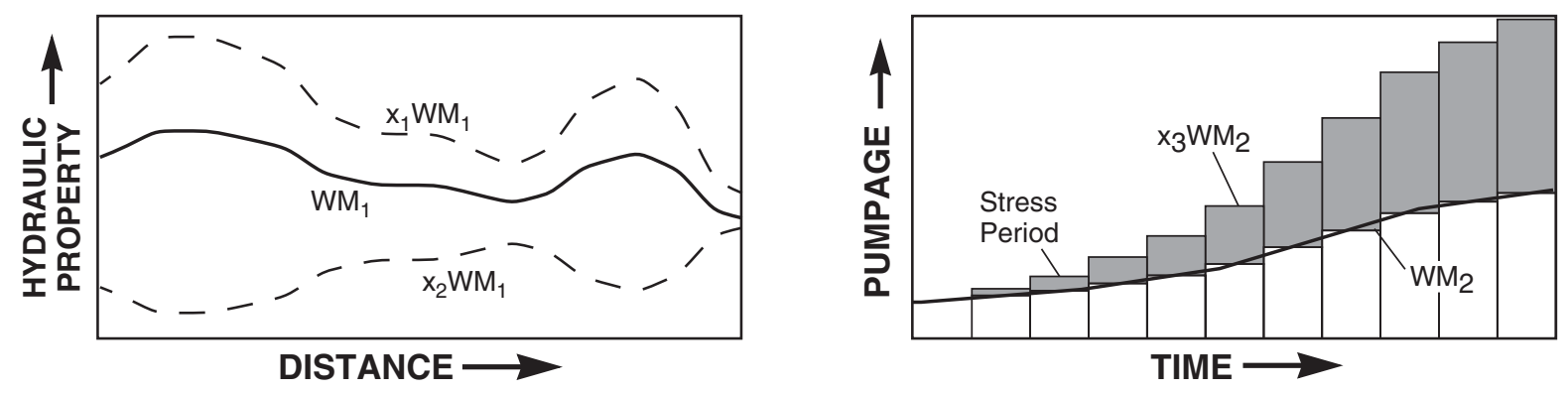

Figure 14. Effect of weight matrices $\mathrm{WM}_{1}$ and $\mathrm{WM}_{2}$ on the spatial variability of a hydraulic property distribution and the temporal variability of a pumpage distribution, respectively.

The weighting specified in one-dimensional and two-dimensional matrices applies equally in unspecified dimensions. For example, a one-dimensional matrix that defines the expected hydraulic-conductivity variability with depth would have one weight per layer that modifies equally all nodes in a layer. Parameter-weight matrices that are defined with an iso-value contour are restricted to two-dimensional matrices. The resulting matrices can be in planes parallel to layers and cross sections. Weights in the matrix are the shortest distances from nodes to appropriate segments of the iso-value contour.

Scaling a parameter-weight matrix reduces unintentional changes in the sensitivity of optimization parameters. Parameter-sensitivity problems arise when values in parameter-weight matrices differ by orders of magnitude. For example, if matrix W1 is equal to 10 times W2. A specified change in an optimization parameter will affect more change in a hydraulic property that is modified with matrix W1 than matrix W2. As a consequence, the optimization algorithm is more sensitive to the optimization parameter defined with matrix W1 than matrix W2. The parameterweight matrix is scaled in MODOPTIM by dividing the initial values in a matrix by the standard deviation of the initial values. 


\section{Optimization Parameters}

Multiple methods of parameter modification are used to scale and constrain optimization parameters. A hydraulic-conductivity distribution might be estimated with exponential modifiers because log-change will be estimated, which constrains estimates to positive values. Whereas, pumpage and injection rates for hydraulic containment might be estimated with an additive modifier because estimates could change sign. Optimization parameters can change aquifer properties and stresses in MODOPTIM as exponential, multiplicative, or additive modifiers. A hydraulic property modified $\left(A P_{M O D}\right)$ at a given node is

$$
\begin{aligned}
& A P_{M O D}=A P_{I N I}\left(\prod_{j=1}^{n} Z M_{j}\right) 10^{M O D} \quad \text { (exponential) } \\
& A P_{M O D}=A P_{I N I}\left(\prod_{j=1}^{n p a r} Z M_{j}\right) M O D \quad \text { (multiplicative), or } \\
& A P_{M O D}=A P_{I N I}\left(\prod_{j=1}^{n p a r} Z M_{j}\right)+M O D \quad \text { (additive). }
\end{aligned}
$$

where:

$A P_{I N I} \quad$ is the initial estimate of the aquifer property or stress in the MODFLOW input file,

$Z M_{j} \quad$ is the zone multiplier for the $j^{\text {th }}$ parameter. If the aquifer property, zone identifier, and stress period are the same as those associated with the $j^{\text {th }}$ parameter, $Z M_{j}$ is equal to the user specified value. Otherwise $Z M_{j}$ is equal to 1 .

$M O D \quad$ is the modifier at a given node and is

where:

$$
\begin{aligned}
& M O D=\sum_{j=1}^{n} x_{j}\left(w m_{j, l}\right) \delta_{j} \text { for parallel summation or } \\
& M O D=1 / \sum_{j=1}^{n p a r} x_{j} \frac{\left(w m_{j, l}\right) \delta_{j}}{x_{j}} \text { for series summation. }
\end{aligned}
$$

$x_{j} \quad$ is the $j^{t h}$ parameter estimated,

$w m_{j, l} \quad$ is the weight from the $l^{\text {th }}$ parameter-weight matrix that was assigned to the $j^{\text {th }}$ parameter estimated. If no parameter-weight matrix was assigned to the $j^{\text {th }}$ parameter, $w m_{j, l}$ is equal to 1 .

$\delta_{j} \quad$ is a Dirac delta function for the $j^{t h}$ parameter. If the aquifer property, zone identifier, and stress period are the same as those associated with the $j^{\text {th }}$ parameter, $\delta_{j}$ is equal to 1 . Otherwise $\delta_{j}$ is equal to 0 .

Optimization parameters are summed in the modifier to incorporate parameter-weight matrices. The simplest use of a parameter-weight matrix requires two optimization parameters that define a constant and a slope. For example, the spatial variability of a horizontal hydraulic-conductivity distribution might be described by a parameterweight matrix of specific-capacity estimates and the optimization parameters would be a multiplier of the parameterweight matrix and a uniform multiplier. Many parameter-weight matrices are used to define a modifier if the parameter-weight matrices represent the estimated fractions of clay, silt, sand, and gravel in model cells (Sun and others, 1995). 
Multiple optimization parameters can define an aquifer property or stress where a zone is the intersection of two or more areas. For example, recharge might be applied to areas A (zones 11 and 13 in fig. 15) and B (zones 12 and 13 in fig. 15) and these two areas overlap (zone 13 in fig. 15). If recharge rates are additive, the recharge distribution can be estimated with two parameters instead of three.

Optimization parameters are summed in parallel (eq. 25) for most problems that define an aquifer property or stress with multiple parameters.

Equation (25) is identified as parallel because the form is the same as that used to add resistances in parallel or sum transmissivities of different lithologies in a volume of bedded aquifer material.

Series summation (eq. 26) of optimization parameters facilitate the estimation of vertical hydraulic-conductivity distributions. The average resistance to flow is approximated as resistors in series when flow moves orthogonally to the bedding structures as occurs through confining units. The average vertical leakance below a cell is the series summation of the weights for the fractions of clay, silt, and sand within the volume of aquifer or confining unit simulated between nodes.

\section{Observations}

All comparisons between simulated and measured observations are defined as either point observations or summations. Water-level and subsidence observations are compared exclusively as point observations. Flow rates are compared as summations over a subvolume. Water-quality and pumping-lift cost observations can be compared as either point observations or summations.

An observation is compared as a point measurement if the simulated response is assumed to be a continuous distribution throughout the simulated volume, such as water level. The continuity assumption allows for interpolation of simulated responses at surrounding nodes to a specified location. The location of a point observation is specified by either cartesian coordinates $\langle\mathrm{XYZ}\rangle$, the column, row, and layer index $<\mathrm{IJK}>$, or a combination of the two coordinate systems. A mixed coordinate system of $\langle\mathrm{XYK}\rangle$ is used frequently to laterally interpolate in the XY plane and specify a layer for comparison.

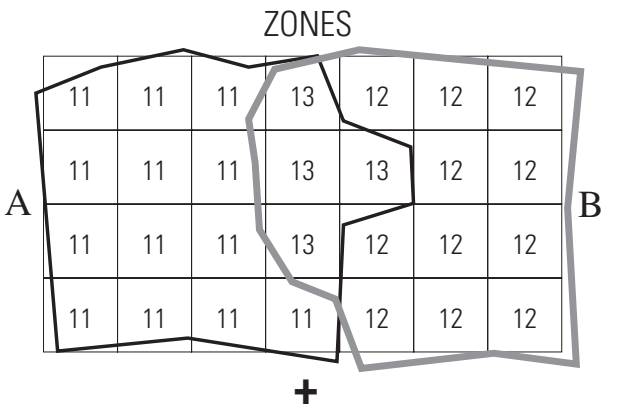

ADDITIVE MODIFIERS

\begin{tabular}{ccc} 
Parameter & Zones & Estimate \\
\hline 1 & 11,13 & 4 \\
2 & 12,13 & 8 \\
\hline
\end{tabular}

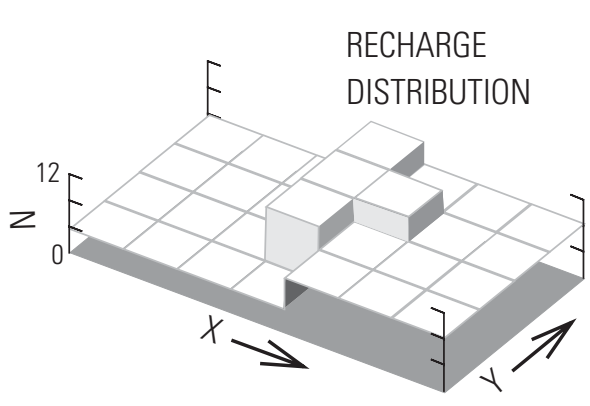

Figure 15. A recharge distribution from two optimization parameters that share a common zone. 
An observation is compared as a summation if the field measurement or optimization goal represents an integrated response from a volume of aquifer system. A comparison between simulated and measured stream discharges would be made as a summation. Total pumping-lift cost from many wells over an area is an optimization goal that is easier to define as a summation over a volume rather than well by well.

\section{Interpolation of Point Observations}

The simulated response at a point observation can be tri-linearly interpolated from surrounding nodes (fig. 16). Functionally, the interpolated response is a weighted summation of the simulated values at surrounding nodes where weights are positive and sum to 1 . Lateral interpolation in the XY-plane is relatively unambiguous except when one or more surrounding nodes lies outside the simulated volume. This condition is addressed by assigning weights of 0 to surrounding nodes that lie outside the simulated volume and multiplying the remaining weights by the inverse of their sum.

Vertical interpolation is dependent on how the aquifer system was conceptualized in addition to model discretization. Hydraulic properties can be conceptualized as being uniform between nodes within layers or as intervening confining units that separate layers. The differences between these two conceptualizations affect the interpolation of both head and subsidence observations. The following discussion will be in terms of head for clarity but applies equally to interpolation of subsidence values from surrounding nodes to a point observation.

If intervening confining units are assumed to separate layers (quasi-3D), the majority of head change between layers is assumed to occur between layers (fig. 17). Vertical interpolation to observations within a layer are not considered because the head is assumed to be vertically uniform within each layer. Vertical interpolation is performed if the observation point lies within an intervening confining unit.

Vertical head change is assumed to be smooth where the resistance to flow is distributed evenly between nodes in layers, 3D (fig. 17). Vertical interpolation is performed linearly between nodes in adjacent layers. The vertical resistance to flow frequently is conceptualized in a 3D manner to simulate partially penetrating wells within an aquifer that is simulated with multiple layers.
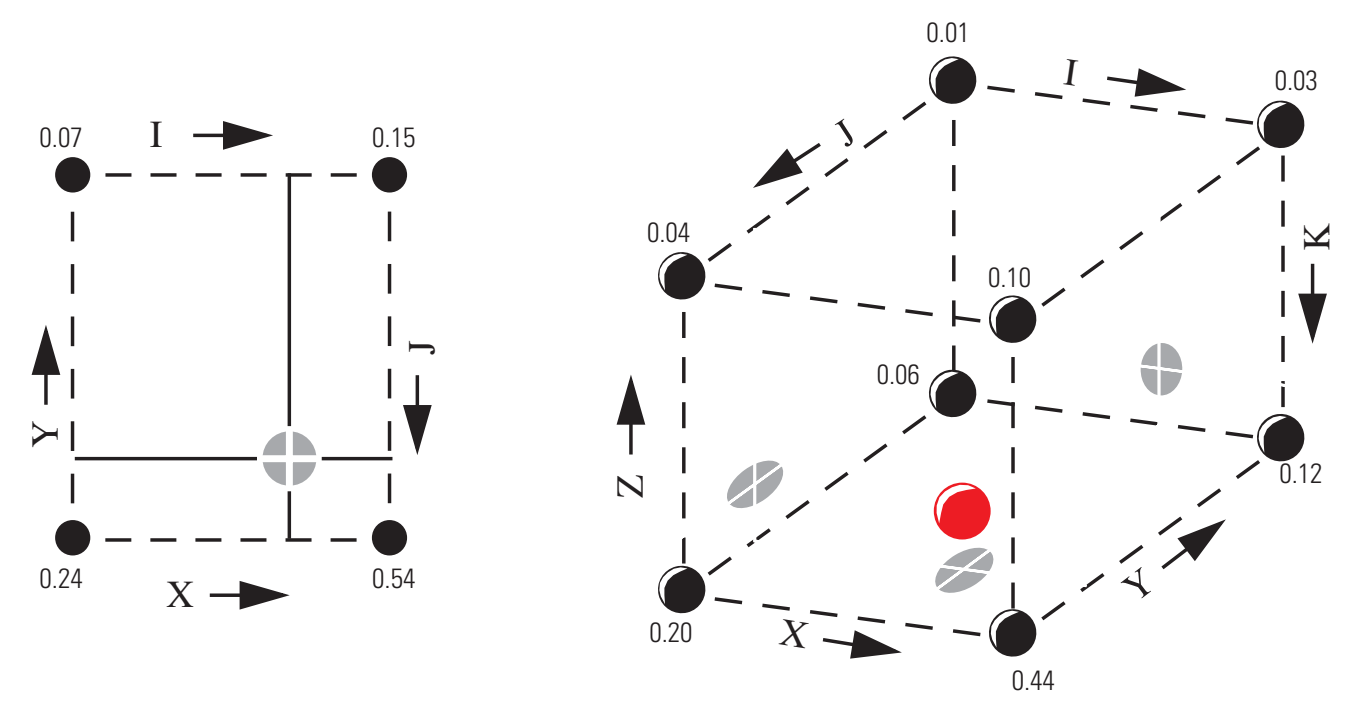

Figure 16. Bi-linear interpolation from the four surrounding nodes in a layer and tri-linear interpolation from the eight surrounding nodes to a point observation. 


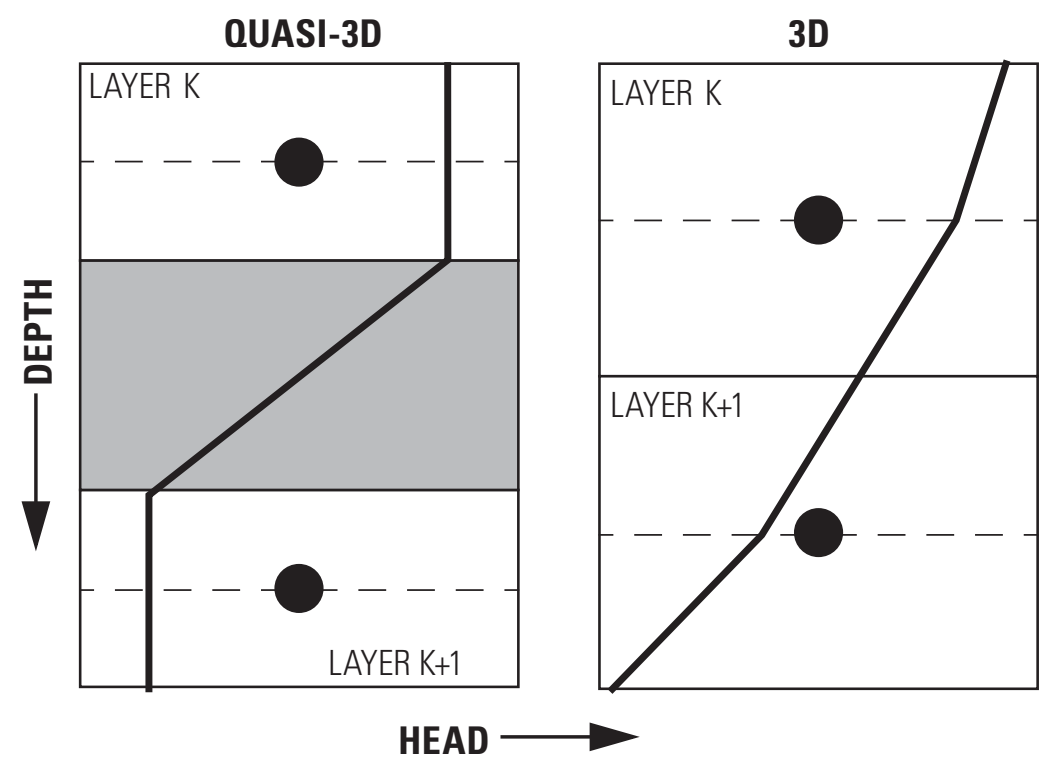

Figure 17. Interpretation of head change between layers in a quasi-3D and $3 \mathrm{D}$ simulation.

\section{Special Cases for Water-Level Observations}

Pumping wells, wellbore storage in observation wells, and rotational translation of observation wells are special comparisons that differ from the standard point comparisons and are applied exclusively to water levels. These special water-level comparisons require additional information and assumptions that can make these observations more useful for general comparison rather than calibration.

\section{Water-Levels in Pumping Wells}

Differences between simulated water levels in a cell and a well are approximated using the Theim equation with an effective external radius (Peaceman, 1983). Wells are assumed to be vertical and coincident with the center of a cell. Flow between the cell and well is assumed to be at steady state. The water-level difference between a cell and a well is simulated by

$$
\Delta h=Q \frac{\ln \left(r_{o} / r_{w}\right)}{2 \pi T}
$$

where:

$Q \quad$ is the discharge rate from a pumping well,

$T \quad$ is the transmissivity of material in cell,

$r_{w} \quad$ is the radius of well casing,

$r_{O} \quad$ is the equivalent external radius defined by Peaceman (1983) where

$$
r_{o}=0.14 \sqrt{\Delta x^{2}+\Delta y^{2}}
$$

$\Delta_{x} \quad$ is the width of the column, and

$\Delta y \quad$ is the width of the row. 


\section{Wellbore Storage in Observation Wells}

Wellbore storage in observation wells is primarily of concern during aquifer tests when rapid water-level responses are analyzed. The high storage coefficient of a wellbore causes water-level responses to lag. This lag between ideal and observed responses becomes more exaggerated for poorly completed wells or wells that are completed in confining units. Neglecting wellbore storage in the observation wells can cause specific storage of an aquifer to be overestimated (Moench, 1997).

Water-level response in an observation well is simulated by

$$
h w_{m}=\frac{\beta \Delta t\left(h_{m}+h_{m-1}-h w_{m-1}\right)+h w_{m-1}}{1+\beta \Delta t}
$$

where:

$h_{m-1}$ is the interpolated water level at the beginning of a time step,

$h_{m}$ is the interpolated water level at the end of a time step,

$h w_{m-1}$ is the water level in the observation well at the beginning of a time step,

$\Delta t \quad$ is the duration of a time step,

$\beta \quad$ is the resistance term, as

$$
\beta=\frac{L_{S C R E E N} K_{X Y}}{2 \ln \left(\frac{L_{S C R E E N}}{r_{w}}\right) r_{w}^{2}}
$$

$K_{X Y} \quad$ is the horizontal hydraulic conductivity of the material around the wellbore,

LSCREEN the length of the well screen, and

$r_{w} \quad$ is the radius of the well casing.

The resistance term, $\beta$, that controls flow between the wellbore and the aquifer is a shape factor from Hvorslev (1951). Water-level lag between a wellbore and the surrounding aquifer is analogous to a slug test.

Simulation of wellbore storage in observation wells is more useful for investigating questions of academic curiosity than it is for improving hydraulic-property estimates from aquifer tests. Potential wellbore storage effects can be tested to bracket the significance of this effect for particular wells. Actively estimating $\beta$ for each observation well will add many parameters to an analysis, and these parameters define local well conditions, not the hydraulic conductivity of an aquifer.

\section{Rotation of Observation Wells}

Lateral anisotropy and orientation can be estimated from aquifer tests that are analyzed with MODFLOW. Ideally, directions of maximum and minimum transmissivity are aligned parallel to the model grid in MODFLOW. Positions of simulated observation wells can be rotated freely about the production well (fig. 18). Rotation is defined in terms of the transmissivity ellipse and is always centered on the production well. A rotation of $+30^{\circ}$ can be interpreted as rotating the transmissivity ellipse $30^{\circ}$ counterclockwise or rotating all of the observation wells $30^{\circ}$ clockwise. Analysis with MODFLOW is advantageous when an aquifer is not Theis-like and leakage from adjacent confining units needs to be simulated. Rotation of observation wells is useful for analyzing aquifer tests.

MODFLOW is applied in a very specific manner to estimate lateral anisotropy and orientation from aquifer test results. A quarter of the domain is simulated because drawdown is symmetrical about the major and minor axes of transmissivity. Production is simulated from row and column $(1,1)$ and is specified as a quarter of the measured rate because only a quarter of the domain is simulated (fig. 19). The production well is centered on the upper, left edge of row and column $(1,1)$. Observation-well locations are redefined within MODOPTIM relative to the production well. Observation-well locations are rotated about the production well and are translated into the simulated area. 
Rotation of transmissivity ellipse

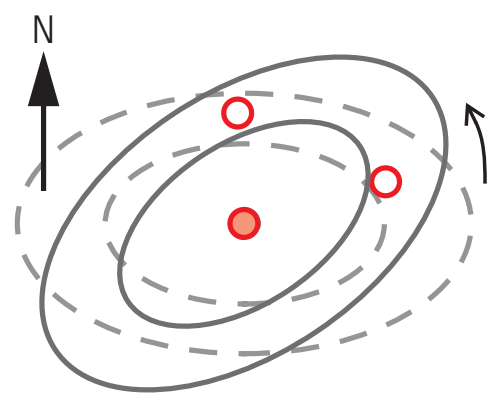

Observation well
Rotation of observation wells

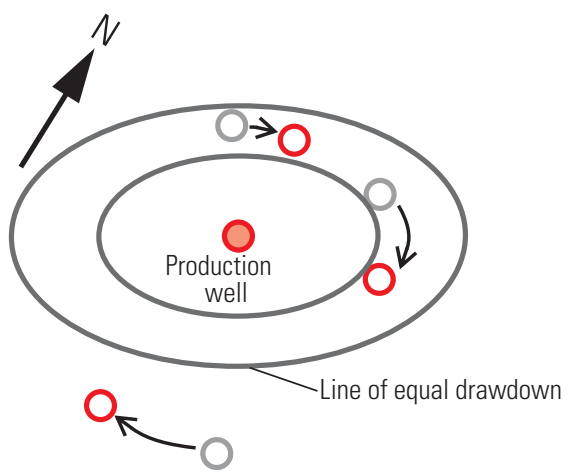

Figure 18. Rotation of lateral transmissivity ellipse or rotation of observation wells about the production well to estimate orientation of lateral transmissivity tensor.

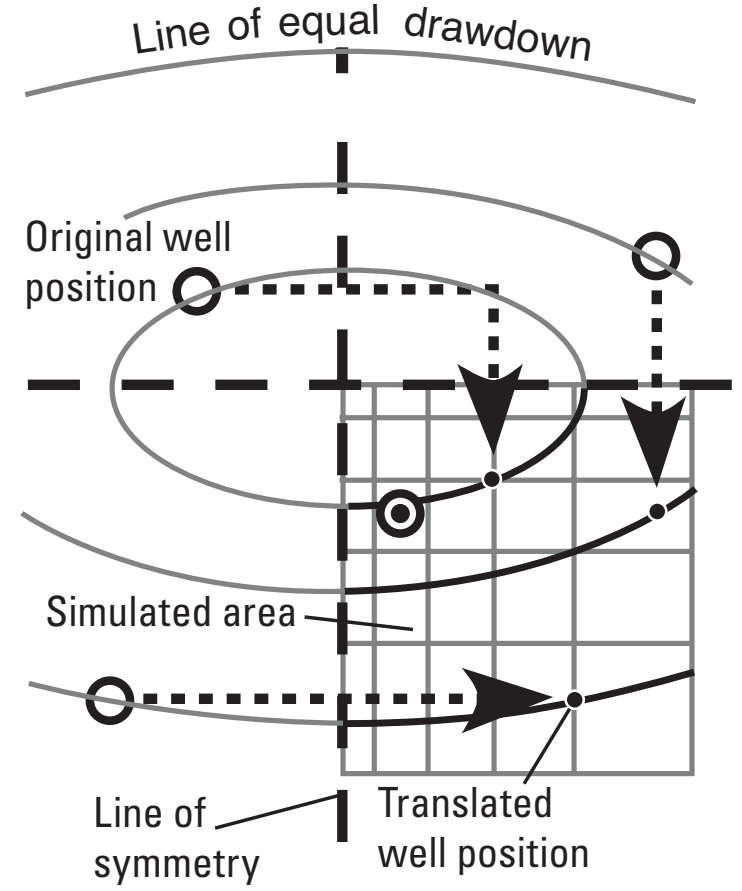

Figure 19. Translation of observation wells to a quarter section.
Rotation of observation wells is defined by a parameter with the TAG:SPIN which cannot be redefined and is in units of degrees. The SPIN parameter should be defined with the additive form (eq. 24). Scaling is needed frequently to make the sensitivity of the SPIN parameter of comparable magnitude to other parameters such as lateral and vertical hydraulic conductivities (Halford and Campbell, 2004).

\section{Subsidence Observations}

Subsidence observations represent the total deformation between the top of the simulated system and the depth of interest for an observation. Subsidence is simulated with the inter-bed storage module (Leake and Prudic, 1991). Land-surface subsidence observations would be compared to simulated results at the base of the model because simulated subsidence is the cumulative compaction of the entire thickness of the aquifer system. Compaction is typically measured using borehole extensometers that may not fully penetrate the aquifer system and cumulative compaction or land-surface subsidence must be extrapolated from the observed compaction.

Many simplified or hypothetical problems can be addressed with one-dimensional or two-dimensional models that have one model layer. For one-dimensional simulations, depth is along the $\mathrm{X}$-axis, column 1 is the top, and column ncol is the bottom. For two- 
dimensional cross sections or radial simulations, depth is along the Y-axis, row 1 is the top, and row nrow is the bottom. Depth in the simulated system is along the Z-axis and compaction is accumulated along layers only for multilayer models.

\section{Flow Observations}

Flow observations are summed across zones that can be the same as those in the IZONE array or defined independently. Zones are elemental building blocks that are grouped together to define a volume. Zones for summing observations can be defined independently of parameter zones to avoid a confusing array of sub-zones that can arise from intersecting dissimilar zoning schemes. Observation zones are stored in the IOBFLX array which is initialized with default values from the IZONE array.

A flow observation can be the summation of discharges from multiple packages that are in the observation zones. Discharges from storage, wells, drains, rivers, streams, evapotranspiration, specified heads, general-head boundaries, and recharge can be compared. For example, simulated discharge at gage A (fig. 20) is the total discharge from the river and drain cells in zones 1 and 2. A flow observation is the summation of all discharges that were identified regardless of sign so simulated discharge can be less than the total flow through the zones of interest.

\section{Optimization Constraints}

Water quality and pumping-lift costs are optimization constraints that are implemented through the MNW package (Halford and Hanson, 2002). Water quality and pumping-lift costs can be defined well-by-well or as summed observations. Water-level constraints on individual wells are specified through the MNW package because minimum discharges and water levels for individual wells must be honored independently of an objective function.

A single water-quality parameter, such as chloride, can be tracked in the MNW package. Water-quality concentrations near pumping wells are specified by the user and are not derived from a solute-transport model. Waterquality concentration is a flow-rate-weighted average that can be from individual wells or user-specified groups of wells.

Pumping-lift costs are assumed to be proportional to the product of lift and discharge (Bredehoeft and Young, 1970). Lift is the difference between a user-specified reference elevation and the pumping water level that is estimated by the MNW package. Discharge is constrained by a minimum pumping water level.

Absolute flow observations serve as a penalty so that total pumpage can be minimized where rates are being estimated for pumping and injection wells. Absolute flow observations are the summation of the absolute value of all flow terms from a specified group of IOBFLX zones.

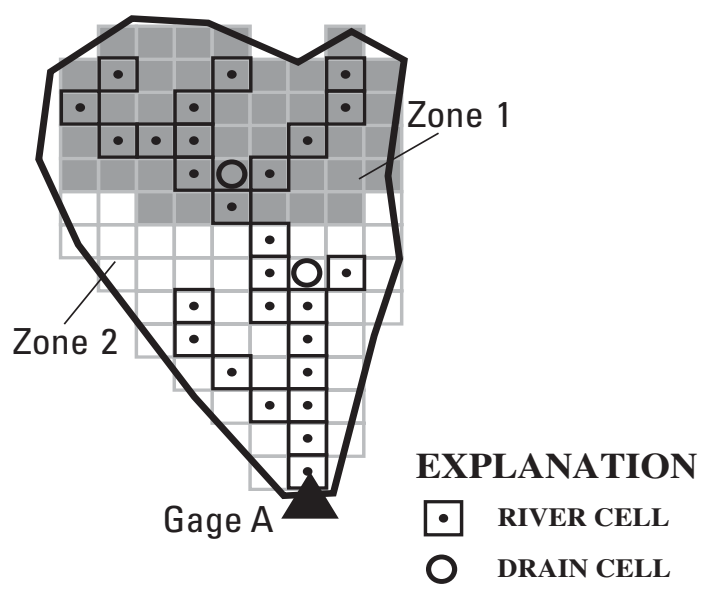

Figure 20. Discharge from river and drain cells that are summed over an area that spans two observation zones. 


\section{Comparison of Observation Differences}

Any observation type can be differenced for comparison. Two observations are specified that differ in space, in time, or in both space and time. Each measured observation is entered and the difference between measured values is computed within MODOPTIM. Raw measured and simulated values are tracked in the first half of the observation pair but are removed from the objective function by weighting the first half to 0 . Observation differences are stored in the second location.

Spatial observation differences are specified by a pointer in the second observation to the site identifier of the first observation. The comparison time is the same as the time when the pointer was set unless stated otherwise. This type of comparison allows for matching vertical water-level differences during parameter estimation and assigning target gradients in ground-water management problems.

Temporal observation differences can be specified as either an offset from a given time (OFFSET) or as differences between each successive measurement (DT). Offset differences are specified by flagging the reference observation that will be subtracted from subsequent observations. Temporal observation differences frequently are used to match the water-level decline in a pumping well during an aquifer test. Water-level differences are matched because late-time changes are controlled by the hydraulic characteristics of the aquifer system, not well-construction or partial-penetration effects. Temporal observation differences also minimize the effects of poor initial conditions on the calibration of transient models.

Sensitivities for observation differences tend to be less than for direct observations and tend to decrease as the distance or elapsed time between observations decreases. Observation differences sometimes need to be weighted more heavily if they are to remain relevant in an objective function. For example, parameter sensitivity differs between water-level and water-level difference observations in a pumping well in a Theis aquifer. The example aquifer has a transmissivity of $1,000 \mathrm{ft}^{2} / \mathrm{d}$, a storage coefficient of 0.0001 , and is pumped for $1 \mathrm{day}$ at $200 \mathrm{gal} / \mathrm{min}$. The sensitivities of the water-level observations are about 10 times greater than the water-level difference observations (fig. 21).

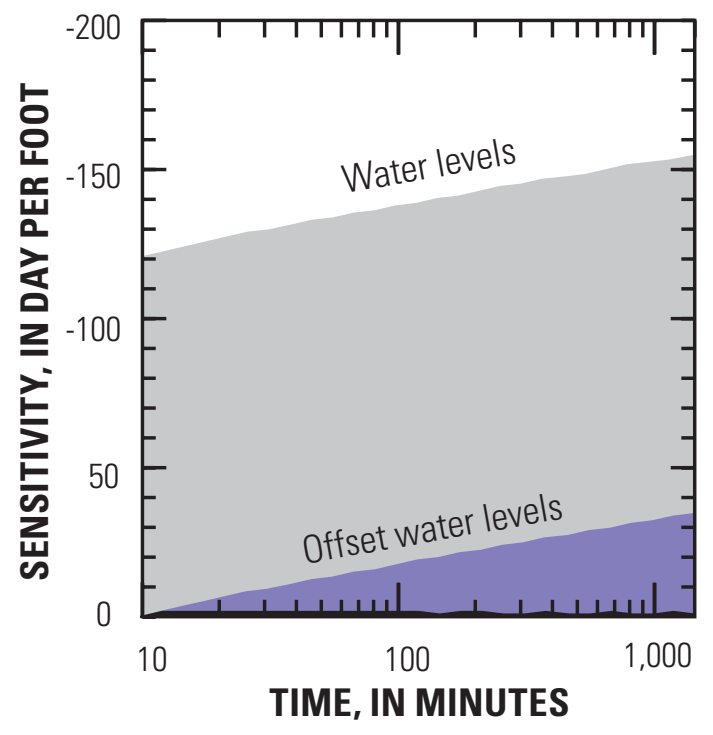

Figure 21. Effect of comparing water levels and water levels from an offset time on sensitivity estimates. 


\section{Weighting of Observations}

The absolute magnitude of observation weights are considered meaningless in MODOPTIM because weights primarily incorporate the opinions of an investigator in an objective function. Weights adjust the relative importance of matching a particular observation type such as water level or discharge and allow for emphasizing more accurate measurements. Many procedures for weighting observations have been postulated that consider measurable errors such as leveling, tape, and discharge accuracy (Watson and others, 1980; Cooley, 1982; Carrera and Neuman, 1986; Hill, 1992; Poeter and Hill, 1997). These procedures create the illusion of a rigorous method for determining observation weights. These rigorous procedures become highly subjective after errors introduced by parameter definition and underlying limiting model assumptions are included, because parameter definition is the primary source of error and is of an unknown magnitude.

Negative and positive residuals are weighted independently so that parameter estimation can use indefinite data, and optimization constraints can be incorporated in objective functions for ground-water management. Independently weighting negative and positive residuals is not relevant for many observations because the weights will be equal. Weights for negative and positive residuals of water levels or drawdowns will be equal because deviations in either direction are equally bad.

Data input is less onerous because multiple default conditions exist. The initial default weight is 1 for both negative and positive residuals of any observation type. Weights can be altered by the entry of a singular value which will be assigned to both negative and positive residuals. Independent weights for negative and positive residuals must be invoked explicitly. Default weights exist for each observation type and can be changed to a new default value with any observation entry.

\section{Inequality Comparisons}

Inequality comparisons are the primary use of independent weights for negative and positive residuals. For example, an optimization constraint might be that spring discharge must be at least $50 \mathrm{ft}^{3} / \mathrm{s}$. This constraint would be imposed by specifying a target discharge of $50 \mathrm{ft}^{3} / \mathrm{s}$ with a non-zero weight for negative residuals and a weight of 0 for positive residuals. Penalizing water tables above land surface is another application of an inequality comparison.

Observations that span a range as opposed to being a single measurement can be defined by inequality comparisons. For example, ground-water discharge "measurements" typically are fuzzy values that are estimated from exceedance plots of surface-water discharge. A pair of discharges are identified that allegedly bracket minimum and maximum ground-water discharges. Simulated discharge from a model that was calibrated with both discharge estimates will lie between the two extremes (fig. 22). This increases the overall model error though "measured" discharge is indeterminate between the minimum and maximum ground-water discharge estimates.

The existence of a range of equally plausible ground-water discharges is incorporated in an objective function as two inequality measurements: one for the minimum discharge with positive residuals weighted to 0 and another for the maximum discharge with negative residuals weighted to 0 (fig. 22). Multiple basins must be included in an objective function for this approach to be reasonable. Calibrating to the discharge of a single basin that has been defined with a pair of inequality measurements will be dependent upon initial parameter estimates and will simulate discharges at either the minimum or maximum extreme.

An objective function defined with many inequality observations will be problematic. The objective function will define a discontinuous error surface which contradicts the assumptions in the optimization algorithm. Some of these problems can be addressed by reducing the weight on inequality observations and allowing the sum-of-square error to increase. Error increases are likely to occur between some iterations if allowed, but model error should decrease on average. 


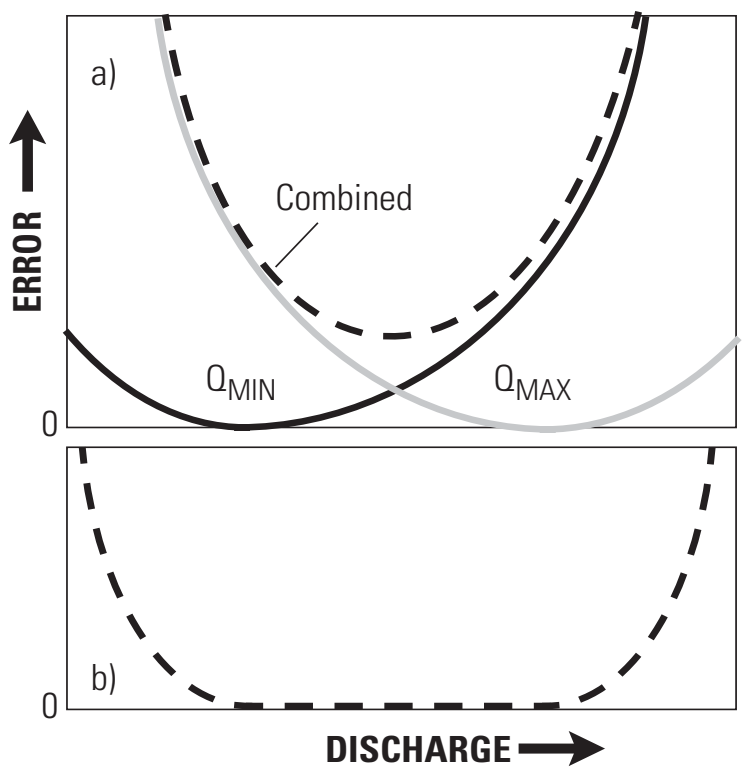

Figure 22. Error response to minimum and maximum ground-water discharge estimates as (a) two independent measurements and (b) a pair of inequality measurements.

\section{Different Observation Types}

Weights are used to account for differences between the units and scales of different observation types that are summed in the composite objective function. The observation type with the largest units or the greatest number of observations will dominate the minimization process. This causes other observation types to be superfluous if weights are not used. In a specific example, unweighted summed-sensitivities for water levels as measured in feet and discharge as measured in $\mathrm{ft}^{3} / \mathrm{s}$ happen to be of similar magnitude. If one uses days instead of seconds as a consistent time unit, unweighted discharge sensitivities become many orders of magnitude greater than the sensitivities of the unweighted water levels.

Assigning weights for different observation types is necessary, but the basis for assigning particular values is ambiguous. Assigning weights that are inversely proportional to observation error is frequently suggested (Watson and others, 1980; Hill and others, 1998). Weights can be adjusted further so that the variance of the weighted residuals for each observation type are equal (Neuman and Yakowitz, 1979; Carrera and Neuman, 1986). Weiss and Smith (1998) introduced three additional methods based on the analysis of parameter space. The ratio of weights between observation types differed by more than seven depending on the weighting method. Weiss and Smith (1998)

concluded, "The decision on which weighting scheme to use will be made by the modeler on the basis of the goals of the model and its intended application."

Assignment of weights based on observation error is not straightforward. Observation error is the sum of model and measurement errors. Measurement error for a water level can be quantified fairly easily but model error for that measurement is difficult to quantify and can be much greater. Model errors are introduced by discretization effects (Poeter and Hill, 1997) and inadequate conceptualization of the aquifer system. Available data are limited for many models, which makes quantification of model error difficult.

The number of observations in each type affects calibration and also needs to be considered while assigning weights. Model error is measured by a sum-of-squares function which does not explicitly account for the number of observations in a given type. Water-level observations will influence parameter estimates more in a problem with 50 water-level measurements for every discharge measurement rather than 20 water-level measurements for every discharge measurement. 
Observation weights are defined subjectively even where all observations are the same type and have the same accuracy. For example, an objective function is defined to minimize the difference between simulated and measured water levels in ten wells (fig. 23). Parameter estimates will be biased toward minimizing the error at site B if all observations are weighted equally. This is a reasonable result if the study investigates small scale variations near site B. A single, average water-level from the wells near site B might be a more reasonable observation for a regional investigation.

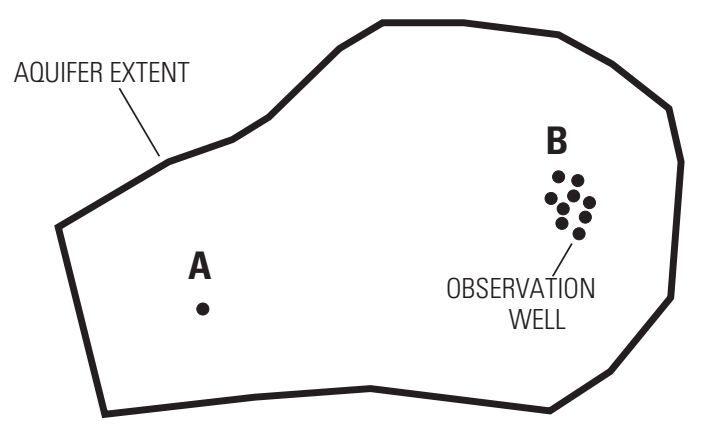

Figure 23. A non-uniform observation well distribution near sites $A$ and $B$.

General statistics are calculated for each observation type in MODOPTIM to provide an initial guide for assigning weights. The standard deviation of the measurements in each observation type can be used to estimate initial weights that will compensate for large differences among units of different observation types. For example, an objective function might incorporate water levels and discharges. Initial weights of $1 \mathrm{can}$ be assigned to water levels while discharges are weighted by the standard deviation of water levels divided by the standard deviation of discharges. In a trial-and-error process, the weights for each observation type can then be adjusted so that each observation type affects the objective function. An observation type typically does not affect calibration where the sum-of-squares of weighted residuals for that type is less than 10 percent of the sum-of-squares of weighted residuals for all observations.

Labels for observation types are arbitrary so statistics for multiple categories of an observation type, such as water levels, can be tracked independently. For example, the model domain could be divided into eastern and western halves. Water levels in the east and west could be labeled as observation types WL-E and WL-W, respectively. Independent statistics of the average, standard deviation, and RMS errors for both weighted and unweighted water levels would be reported for the eastern and western halves of the model domain.

\section{Output and Analysis of Results}

Results from MODOPTIM are directed to a master optimization output file, a parameter-estimate file, and a residual-and-sensitivities file. Input from the OPT file is echoed to the master optimization output file along with parameter estimates from each optimization iteration. Simulated observations, measured observations, weights, residuals, and weighted-sensitivity coefficients for the simulation with the smallest error are reported to the residualand-sensitivity file. The best set of parameter estimates and statistics for evaluating optimization results are summarized in the parameter-estimate file.

Several MODFLOW input files also are generated as output from MODOPTIM. MODFLOW files that are generated by MODOPTIM can be used with other programs such as MODPATH or saved for archival purposes. BAS and BCF files are always generated by MODOPTIM. Additional files are processed through MODOPTIM as additional parameters from packages other than the BCF package are estimated. 


\section{Parameter Estimates and Optimization Results}

Optimization statistics and parameter estimates with the smallest sum-of-squares error are summarized for the model in the parameter-estimate file. Weighted sum-of-squares, RMS, and average errors are reported for the complete objective function and by observation type. Unweighted error statistics also are reported. Parameter estimates are ranked and reported by relative sensitivity.

The robustness of an objective function to a parameter can be evaluated with relative sensitivity and weightedsensitivity coefficients of individual observations. Parameters with relative sensitivities greater than 0.1 generally are estimated well by that objective function. This is not the case where the relative sensitivity has been influenced unduly by a few observations. A large weighted-sensitivity coefficient for an observation can skew the relative sensitivity for a parameter and typically results from heavily weighting that observation. Heavily weighted observations can be detected in the parameter estimate summary where the first and second most sensitive observations for each parameter are reported.

Interdependence of parameter pairs can be evaluated with a correlation coefficient and a measure-ofredundancy. Correlation coefficients are normalized elements in the inverse of the Hessian matrix, $\left[J_{T} J\right]^{-1}$, elements along the main diagonal equal 1 and off-diagonal elements range from -1 to 1 . Correlation coefficients describe parameter interdependence in the context of all parameters estimated and will be affected by changing the number of parameters estimated. A correlation coefficient greater than 0.99 for a pair of parameters indicates two parameters cannot be estimated independently (Poeter and Hill, 1997).

A measure-of-redundancy (MOR) describes how similar two parameters appear to a given objective function.

MOR between the $i^{\text {th }}$ and $j^{\text {th }}$ parameters is $M O R_{i, j}=\frac{\sum_{n, i} J_{n, j}}{\sqrt{\sum_{n=1}^{n o b s} J_{n, i}^{2} \sum_{n=1}^{n o b s} J_{n, j}^{2}}}$ and values range from -1 to 1. MOR

values greater than 0.99 also indicate parameter pairs are correlated highly. The MOR statistic is dependent upon sensitivities computed for just the two parameters and will not be affected by changing the number of parameters estimated.

A wider range of problems can be detected with both correlation coefficients and MOR. Correlation coefficients can identify many parameter pairs that are highly correlated but fail to identify a specific parameter pair that causes the high degree of correlation. The parameter pair with the greatest MOR typically contains the parameter that should be eliminated.

\section{Residuals and Sensitivities}

Information for assessing calibration and optimization results are summarized in the residual-and-sensitivity file. For each observation, time of observation, measured value, simulated value, residual, weight, spatial coordinates, and weighted-sensitivity coefficients to each parameter are reported for the model with the smallest sum-of-squares error. Spatial coordinates are not reported for observations that are summed across zones, such as discharge.

Hydrographs, scatter plots, and maps of residuals can be created easily from results in the residual-andsensitivity file. Goodness-of-fit can be evaluated qualitatively and systematic trends in residuals can be identified with these plots and maps. Hydrographs of water levels and discharges allow for comparing simulated trends to measured trends. Scatter plots of measured and simulated observations are useful for evaluating overall goodness-of-fit relative to the range of measured observations. Systematic patterns in residuals can be identified with mapped values. Plots and maps of unweighted values provide a clear perspective of goodness-of-fit that has not been biased by estimated weights.

Observations that significantly influence parameter estimates are readily identified with weighted-sensitivity coefficients (eq. 21). A weighted-sensitivity coefficient exists for each observation and each estimated parameter, so 1,000 weighted-sensitivity coefficients exist for a problem with 100 observations and 10 estimated parameters. Bar- 
charts of observation number and weighted-sensitivity coefficients for each parameter are good for identifying patterns and problematic observations. For example, weighted-sensitivity coefficients for 214 observations from 6 wells to transmissivity changes in a Theis aquifer show increases in sensitivity with time and decreases in sensitivity with distance (fig. 24). No single observation from this example would dominate parameter estimation.

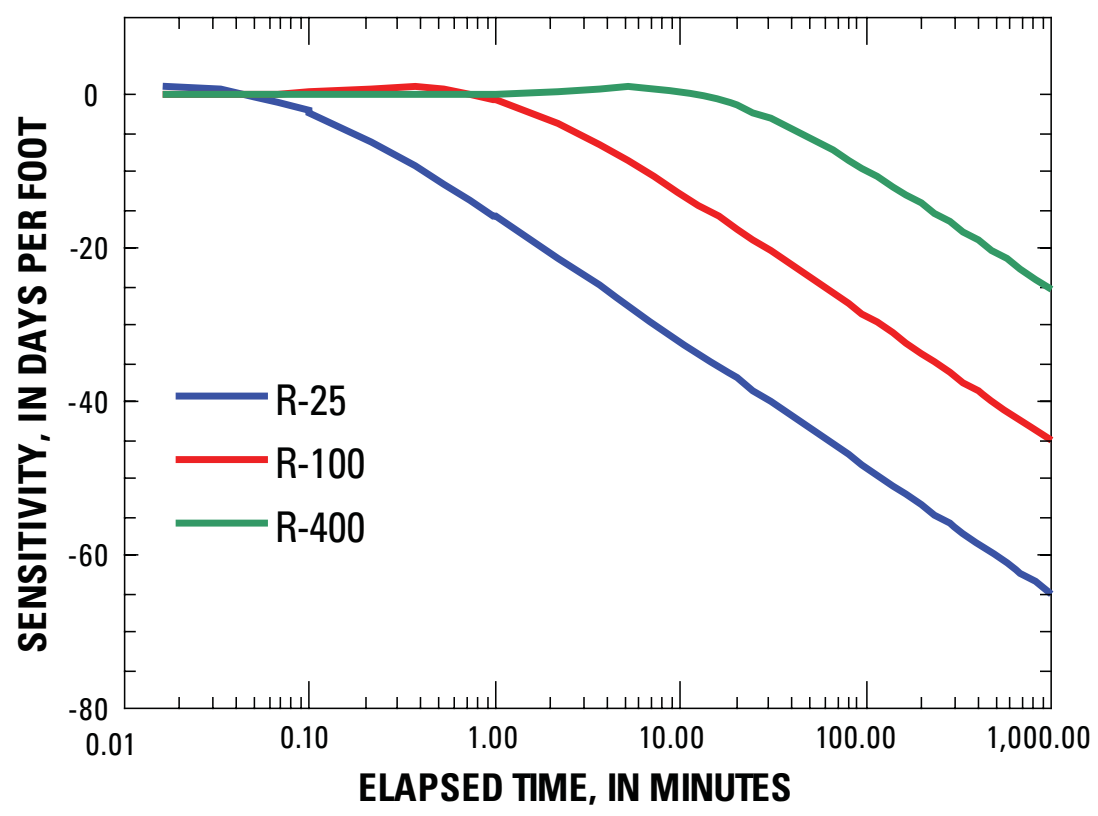

Figure 24. Weighted sensitivities for transmissivity change in a Theis aquifer at radii of 25,100 , and 400 feet from the production well.

Standard plots of parameter change and RMS error can be generated easily with MODOPTIM. These plots are referred to as U-plots in this publication because of the characteristic ' $U$ ' shape of model error about calibrated parameter estimates (figs. 9 and 22). A 'U' of RMS error is computed at user-specified points about the initial value of each parameter. U-plot computation is performed instead of optimizing when this option is selected (Appendix B, Data Group 2).

Model sensitivity to water levels at unobserved locations and times can be investigated easily without creating many bogus observations. Water-level differences are proportional to sensitivities for a parameter and are reported instead of sensitivities because water-level differences are easier to explain and comprehend. Water-level difference distributions can be created for each parameter estimated throughout the model domain. Water-level differences are the differences between results from an initial model and results from a model with a parameter perturbed by the influence coefficient (CFI). Water-level differences are stored for all layers as specified by the output-control file for each specified parameter (fig. 25). An unformatted head file is created for each specified parameter. Water-level difference distributions are computed instead of optimization when the specified maximum number of iterations (MAXIT) is less than 0 (Appendix B, Data Group 2). 


\begin{tabular}{|c|c|c|c|c|c|c|c|c|}
\hline & 1 & 2 & 3 & 4 & 5 & 6 & 7 & 8 \\
\hline 1 & -0.30 & -0.30 & -0.31 & -0.32 & -0.33 & -0.33 & -0.32 & -0.32 \\
\hline 2 & -0.32 & -0.33 & -0.34 & -0.36 & -0.37 & -0.37 & -0.36 & -0.36 \\
\hline 3 & -0.35 & -0.37 & -0.41 & -0.45 & -0.50 & -0.47 & -0.43 & -0.41 \\
\hline 4 & -0.38 & -0.42 & -0.49 & -0.61 & -0.84 & -0.62 & -0.52 & -0.47 \\
\hline 5 & & & -0.52 & -0.56 & -0.60 & -0.57 & -0.53 & \\
\hline
\end{tabular}

Figure 25. Sensitivity of water levels in a layer to a pumpage change in cell $(4,5)$.

\section{COMPARISON TO OTHER PROGRAMS}

MODOPTIM differs from other parameter estimation programs such as UCODE (Poeter and Hill, 1998), PEST (Doherty, 2004), and MODFLOW-2000 (Hill and others, 2000). Automatic elimination of insensitive parameters and inequality comparisons are exclusive features of MODOPTIM. Wellbore storage effects in observation wells and rotation of observation wells are not supported by UCODE, PEST, or MODFLOW-2000. These features could be implemented with PEST or UCODE, but the user would need to program the functions. Regularized pilot points is a useful feature of PEST that is not supported in MODFLOW-2000, MODOPTIM, or UCODE.

Many features of MODOPTIM are specific to aquifer test analyses and are not supported by UCODE (Poeter and Hill, 1998), PEST (Doherty, 2004), or MODFLOW-2000 (Hill and others, 2000). MODOPTIM will create BCF files that simulate axisymmetric, radial flow. Transmissivity, vertical leakance, and storage of the $i^{\text {th }}$ column are multiplied by $2 \pi r_{i}$, where $r_{i}$ is the distance from the outer edge of the first column to the center of the $i^{t h}$ column. Wellbore storage in observation wells is simulated. Lateral anisotropy of aquifers in geologically complex systems can be estimated by rotation of observation wells.

MODOPTIM optimizes ground-water management with non-linear programing because a wider class of problems can be solved than with linear programming as used in MODOFC (Ahlfeld and Riefler, 1999). A non-linear approach requires more computation but less user interaction. MODOPTIM is not as versatile a ground-water management tool as MGO (Zheng and Wang, 2002), which uses heuristic search techniques such as simulated annealing, genetic algorithms, and tabu search.

\section{REFERENCES}

Ahlfeld, D.P., and Riefler, R.G.,1999, Documentation for MODOFC: A program for solving optimal flow control problems based on MODFLOW simulation, version 2.11, accessed November 15, 2005, at http://www.ecs.umass.edu/modofc/

Barrash, W., and Dougherty, M.E., 1997, Modeling axially symmetric and nonsymmetric flow to a well with MODFLOW, and application to Goddard2 well test, Boise, Idaho: Ground Water, v. 35, no. 4, p. 602-611.

Bredehoeft, J.D., and Young, R.A., 1970, The temporal allocation of ground water-A simulation approach: Water Resources Research, v. 6, no. 1, p. 3-21.

Carrera, Jesus, and Neuman, S.P., 1986, Estimation of aquifer parameters under transient and steady-state conditions: 2. Uniqueness, stability, and solution algorithms: Water Resources Research, v. 22, no. 2, p. 211-227.

Chau, T.S., 1992, Optimal management of relief wells near Waterton reservoir: Water Resources Bulletin, v. 28, no. 2, p. 349-360.

Chavent, G., Dupuy, M., and Lemonnier, P., 1975, History matching by use of optimal theory: Society of Petroleum Engineers Journal, v. 15 , no. 1 , p. $74-86$.

Chen, W.H., Gavalas, G.R., Seinfeld, J.H., and Wasserman, M.L., 1974, A new algorithm for automatic history matching: Society of Petroleum Engineers Journal, v. 14, no. 6, p. 593-608. 
Coats, K.H., Dempsey, J.R., and Henderson, J.H., 1970, A new technique for determining reservoir description from field performance data: Society of Petroleum Engineers Journal, v. 10, no. 1, p. 66-74.

Cooley, R.L., 1977, A method of estimating parameters and assessing reliability for models of steady state groundwater flow, 1 , Theory and numerical properties: Water Resources Research, v. 13, no. 2, p. 318-324.

Cooley, R.L., 1982, Incorporation of prior information into nonlinear regression groundwater flow models, 1, Theory: Water Resources Research, v. 18, no. 4, p. 965-976.

Cooley, R.L., 2004, A theory for modeling ground-water flow in heterogeneous media: U.S. Geological Survey Professional Paper 1679, $220 \mathrm{p}$.

Doherty, J., 2003, Groundwater model calibration using pilot points and regularisation: Ground Water, v. 41 no. 2, p. 170-177.

Doherty, J., 2004, PEST: Model-Independent Parameter Estimation (5th ed.): Corinda, Australia, Watermark Numerical Computing, $336 \mathrm{p}$.

Dougherty, D.E., and Marryott, R.A., 1991, Optimal groundwater management: 1. Simulated annealing: Water Resources Research, v. 27, no. 10, p. 2493-2508.

Freyberg, D.L., 1988, An exercise in ground-water model calibration and prediction: Ground Water, v. 26, no. 3, p. 350-360.

Gill, P.E., Murray W., and Wright, M.H., 1981, Practical optimization: San Diego, Calif., Academic Press, 401 p.

Gorelick, S.M., 1983, A review of distributed parameter groundwater management modeling methods: Water Resources Research, v. 19, no. 2, p. 305-319.

Gorelick, S.M., and Wagner, B.J., 1986, Ground-water-flow modeling and optimization techniques applied to high ground-water problems in San Bernardino, California, in S. Subitzky, ed., Selected papers in the hydrologic sciences: U.S. Geological Survey Water-Supply Paper 2290, p. 81-89.

Halford, K.J., 1992, Incorporating reservoir characteristics for automatic history matching: Baton Rouge, La., Louisiana State University, Ph.D. dissertation, $150 \mathrm{p}$.

Halford, K.J., 1998, Ground-water flow in the surficial aquifer system and potential movement of contaminants from selected waste-disposal sites at Naval Station Mayport, Florida: U.S. Geological Survey Water-Resources Investigations Report 97$4262,104 \mathrm{p}$.

Halford, K.J., and Campbell, B.C., 2004, A unique approach to estimating lateral anisotropy in complex geohydrologic environments: Journal of Hydraulic Research, v. 42, extra issue, p. 70-79.

Halford, K.J., and Hanson, R.T., 2002, User guide for the drawdown-limited, multi-node well (MNW) package for the U.S. Geological Survey's modular three-dimensional finite-difference ground-water flow model, versions MODFLOW-96 and MODFLOW 2000: U.S. Geological Survey Open-File Report 02-293, 33 p.

Halford, K.J., and Lovelace, J., 1994, Analysis of ground-water flow in the "1,200-foot" aquifer, Baton Rouge area, Louisiana: Louisiana Department of Transportation and Development, Office of Public Works Water Resources Technical Report, Technical Report No. 54, 68 p.

Harbaugh, A.W., and McDonald, M.G., 1996, Programmer's documentation for MODFLOW-96, an update to the U.S. Geological Survey modular finite-difference ground-water flow model: U.S. Geological Survey Open-File Report 96-486, 220 p.

Hill, M.C., 1992, A computer program (MODFLOWP) for estimating parameters of a transient, three-dimensional, ground-water flow model using non-linear regression: U.S. Geological Survey Open-File Report 91-484, 358 p.

Hill, M.C., 1998, Methods and guidelines for effective model calibration: U.S. Geological Survey Water-Resources Investigations Report 98-4005, $90 \mathrm{p}$.

Hill, M.C., Banta, E.R., Harbaugh, A.W., and Anderman, E.R., 2000, MODFLOW-2000, the U.S. Geological Survey modular ground-water model-User guide to the observation, sensitivity, and parameter-estimation processes and three postprocessing programs: U.S. Geological Survey Open-File Report 00-184, 210 p.

Hill, M.C., Cooley, R.L., and Pollock, D.W., 1998, A controlled experiment in ground water flow model calibration: Ground Water, v. 36, no. 3, p. 520-535.

Holmes, J.K., Chu, Wen-sen, and Erickson, D.R., 1989, Automated calibration of a contaminant transport model for a shallow sand aquifer: Ground Water, v. 27, no. 4, p. 501-508.

Hvorslev, J.M., 1951, Time lag and soil permeability in ground-water observations: U.S. Army Corps of Engineers Waterways Experiment Station, Vicksburg, Miss., Bulletin 36, 50 p.

Jacquard, P., and Jain, C., 1965, Permeability distribution from field pressure data: Society of Petroleum Engineers Journal, v. 5 , no. 4, p. 281-294.

Jahns, H.O., 1966, A rapid method for obtaining a two-dimensional reservoir description from well response data: Society of Petroleum Engineers Journal, v. 6, no. 4, p. 315-327.

Kuiper, L.K., 1986, A comparison of several methods for the solution of the inverse problem in two-dimensional steady state groundwater flow modeling: Water Resources Research, v. 22, no. 5, p. 705-714. 
Leake, S.A., and Prudic, D.E., 1991, Documentation of a computer program to simulate aquifer-system compaction using the modular finite-difference ground-water flow model: U.S. Geological Survey Techniques of Water-Resources Investigations, book 6, chap. A2, 68 p.

Marquardt, D.W., 1963, An algorithm for least-squares estimation of nonlinear parameters: Journal of the Society for Industrial Applied Mathematics, v. 11, no. 2, p. 431-441.

McDonald, M.G., and Harbaugh, A.W., 1988, A modular three-dimensional finite-difference ground-water flow model: U.S. Geological Survey Techniques of Water-Resources Investigations, book 6, chap. A1, $576 \mathrm{p}$.

Moench, A.F., 1997, Flow to a well of finite diameter in a homogeneous, anisotropic water table aquifer: Water Resources Research, v. 33, no. 6, p. 1397-1407.

Neuman, S.P., and Yakowitz, S., 1979, A statistical approach to the inverse problem in aquifer hydrology, 1, Theory: Water Resources Research, v. 15, no. 4, p. 845-860.

Peaceman, D.W., 1983, Interpretation of well-block pressures in numerical reservoir simulation with nonsquare grid blocks and anisotropic permeability: Society of Petroleum Engineers Journal, p. 531-543.

Poeter, E.P., and Hill, M.C., 1997, Inverse models: A necessary next step in ground-water modeling: Ground Water, v. 35, no. 2 , p. 250-260.

Poeter, E.P., and Hill, M.C., 1998, Documentation of UCODE, a computer code for universal inverse modeling: U.S. Geological Survey Water-Resources Investigations Report 98-4080, 116 p.

Reichard, E.G., 1995, Groundwater-surface water management with stochastic surface water supplies: A simulation optimization approach: Water Resources Research, v. 31, no. 11, p. 2845-2865.

Shah, P.C., Gavalas, G.R., and Seinfeld, J.H., 1978, Error analysis in history matching: The optimum level of parameterization: Society of Petroleum Engineers Journal, p. 219-228.

Sun, N.Z., Jeng, M.C., and Yeh, W.W.-G., 1995, A proposed geological parameterization method for parameter identification in three-dimensional groundwater modeling: Water Resources Research, v. 31, no. 1, p. 89-102.

Thomas, L.K., Hellums, L.J., and Reheis, G.M., 1971, A nonlinear automatic history matching technique for reservoir simulation models, Paper SPE 3475 presented at the 46th Annual Fall Meeting of the Society of Petroleum Engineers, New Orleans, La., October 3-6, 1971.

Townley, L.R,. and Wilson, J.L., 1985, Computationally efficient algorithms for parameter estimation and uncertainty propagation in numerical models of groundwater flow: Water Resources Research, v. 21, no. 12, p. 1851-1860.

Veatch, Jr., R.W., and Thomas, L.K., 1971, A direct approach for history matching, Paper SPE 3515 presented at the 46th Annual Fall Meeting of the Society of Petroleum Engineers, New Orleans, La., October 3-6, 1971.

Vecchia, A.V., and Cooley, R.L., 1987, Simultaneous confidence and prediction intervals for nonlinear regression models with application to a groundwater flow model: Water Resources Research, v. 23, no. 7, p. 1237-1250.

Wasserman, M.L., Emanuel, A.S., and Seinfeld, J.H., 1975, Practical applications of optimal-control theory to history-matching multiphase simulator models: Society of Petroleum Engineers Journal, p. 347-355.

Watson, A.T., and Lee, W.J., 1986, A new algorithm for automatic history matching production data, Paper SPE 15228 presented at the Unconventional Gas Technology Symposium, Louisville, Ky., May 18-21, 1986.

Watson, A.T., Seinfeld, J.H., Gavalas, G.R., and Woo, P.T., 1980, History matching in two-phase petroleum reservoirs: Society of Petroleum Engineers Journal, p. 521-532.

Weiss, R., and Smith L., 1998, Efficient and responsible use of prior information in inverse methods: Ground Water, v. 36, no. 1, p. 151-163.

Yager, R.M., 2003, Calculation of model sensitivity and its effect on nonlinear regression of ground-water flow, in MODFLOW and More 2003: Understanding through Modeling, Proceedings of the 5th International Conference of the International Ground Water Modeling Center: Golden, Colo., Colorado School of Mines, p. 580-584.

Yang, P.H., Armasu, R.V., and Watson A.T., 1987, Automatic history matching with variable-metric methods, Paper SPE 16977 presented at the 62nd Annual Technical Conference and Exposition, Dallas, Tex., September 27-30, 1987.

Yeh, W.W.-G., 1986, Review of parameter identification procedures in groundwater hydrology: Water Resources Research, v. 22, no. 2, p. 95-108.

Yeh, W.W.-G., 1992, Systems analysis in ground-water planning and management: American Society of Civil Engineers Journal of Water Resources Planning and Management, v. 118, no. 3, p. 224-237.

Yeh, W.W.-G., and Yoon, Y.S., 1981, Aquifer parameter identification with optimum dimension in parameterization: Water Resources Research, v. 17, no. 3, p. 664-672.

Zheng, Chunmiao, and Wang, P.P., 2002, MGO: A modular groundwater optimizer incorporating MODFLOW/MT3DMS, documentation and user's guide: Tuscaloosa, Ala., University of Alabama, $118 \mathrm{p}$. 


\section{Appendix A Input structures used in MODOPTIM files}

Data are read from MODOPTIM input files as 2,048-character alphanumeric cards to facilitate the addition of comments within the model input files and the use of keys to identify input variables. All integer, real, and character variables are read from the alphanumeric cards. The cards are initially read by the subroutine NCREAD. Cards with a '\#' sign in the first column are treated as comment cards, are not passed to any other routines, and are discarded. Once NCREAD has acquired a valid data card, the card is checked for a '!' sign. If a '!' sign is detected, the '!' sign and all text right of the '!' sign are removed from the card before passing it to any other routines.

The subroutine QREAD extracts numeric values as real numbers from the input cards acquired by NCREAD and will read all numeric values that are followed by a trailing blank or comma. Numeric values will be read from the card regardless of the presence or absence of text on the card which allows for text only descriptors to be embedded next to the input variables. All integer variables are read as real numbers and converted to integers to avoid reading errors if the user specifies the variable as a real number.

The typical functioning of the subroutines NCREAD and QREAD are best illustrated by example. If the following is read from an input file by NCREAD:

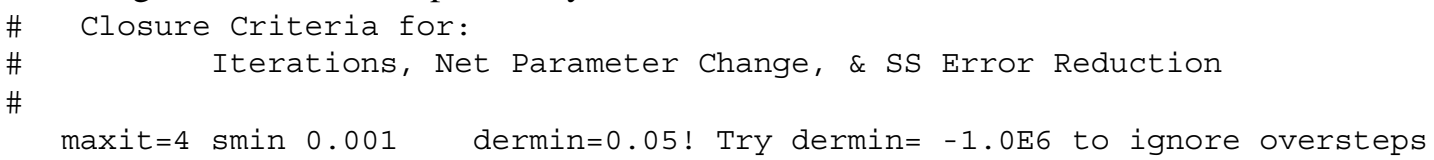

\#

NCREAD returns the stripped input card (maxit $=4 \operatorname{smin} 0.001$ dermin $=0.05)$ to the routine and the subroutine QREAD extracts three values (4, 0.001, and 0.05).

Array data are read with the subroutine GETMAT, which utilizes the subroutines NCREAD and QREAD. As such, arrays can contain comment cards and text identifiers within the field of the array but all numeric entries must be separated by blank, comma, or tab delimiters. Row numbers can be denoted in the input by placing the values beyond the rightmost edge of the matrix and preceding the value with a '!' sign. Array data entry is terminated with an '<end >' flag.

Alphanumeric strings are used in MODOPTIM to identify variables (keys) and logical conditions (flags). Specification of these keys and flags is case insensitive because all letters are capitalized before performing any logical tests. Keys precede the variable to be read. Logical decisions are based on the presence (true) or absence (false) of a flag. Data entry with key:data pairs will be presented as follows:

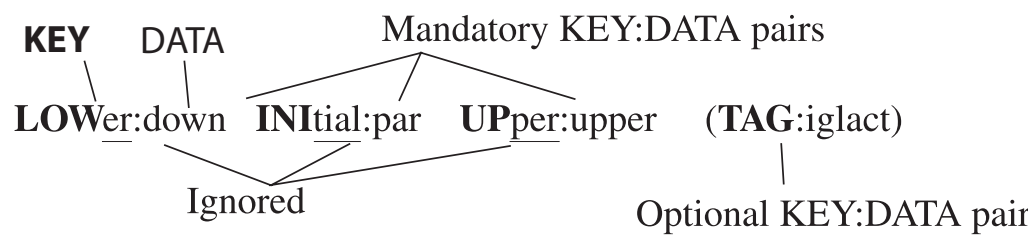

where:

Bold, upper-case letters denote the part of the key that is tested by MODOPTIM,

Key:data pairs that are not delimited by parentheses are mandatory and must be included, and

Key:data pairs that are delimited by parentheses are optional; default values exist if they are not specified.

\section{I/O Redirection}

The three primary MODOPTIM files (Appendices B, C, and D) can be subdivided into smaller files with I/O redirection. I/O can be redirected from the primary file to an auxiliary input file by inserting an I/O redirect statement at any location in the file. Multiple I/O redirect statements can be used in a file. I/O redirection can only occur from a primary MODOPTIM file and not from an auxiliary input file. 
1. DATA:

TYPE:
REDIRECT

Alphanumeric header card
:Filename

REDIRECT:Filename=The filename of the auxiliary input must follow the colon. The REDIRECT key switches the I/O from the primary file to the auxiliary file. All eight characters must be present. Sample input of an element of GROUP 1 data:

\#

Tag: PERM Layer: 1

$\begin{array}{lll}10 & 1.000 & (6 e 13.5)\end{array}$

Redirect to file:sem_bos.array

Tag: VCON Layer: 1

$\begin{array}{llll}0 & 1.000 & (6 e 13.5) & -7\end{array}$
I/O redirect occurs here 


\section{Appendix B Input for main optimization file}

DATA GROUP $1-$ FILE SPECIFICATIONS

Read 6 lines with file name and unit number that specify:

1A. The composite MODFLOW/MODOPTIM file for building BAS, BCF, and other MODFLOW files with parameters to be estimated.

1B. $\quad$ Main MODOPTIM output file.

1C. MODOPTIM update file that contains residuals and sensitivities.

1D. Name of MDFLOW-BAS file that is generated by MODOPTIM.

1E. $\quad$ Name of standard MODFLOW output file.

1F. $\quad$ MODOPTIM update file that contains best parameter estimates.

DATA GROUP 2 - CLOSURE CRITERIA FOR OPTIMIZATION

NOTE: Cards 2A and 2B are mutually exclusive.

2A. DATA: Maxit Smin Dermin CCmin

TYPE: $\quad$ Integer Real Real Real

Maxit = Maximum number of parameter estimation iterations

If Maxit $<0$, sensitivity arrays for each optimization parameter are computed and optimization is not performed.

Smin = Minimum overall parameter change

Dermin $=$ Minimum reduction in sum-of-squares objective function

$\mathrm{CCmin}=$ All parameter pairs with correlation coefficients greater than CCmin are explicitly shown in the MODOPTIM parameter-estimate file (PAREST).

2B. DATA: $\quad$ SENSITIVITY PLOT Points Multiply

TYPE: $\quad$ Alphanumeric header card

SENSITIVITY PLOT a flag that causes U-plot sensitivity profiles to be calculated for each active parameter and causes optimization to not be performed.

Points $=$ Number of RMS points to be calculated along an error profile.

Multiply $=$ Defines range of parameter profile as a multiple of the initial value. For example, the sensitivity profile for a parameter with an initial value of 20 and Multiply equal to 5 would range from 4 to 100.

DATA GROUP 3 - OPTIMIZATION CONTROL VARIABLES

3. DATA: $\quad$ Smax $\quad$ Stfrc Fresec Rkmin $\quad$ Cfi(LIMIT:Choice)

TYPE: Real Real Real RealReal Logical choice

Smax $=$ the maximum step length for all parameters. A typical value is 0.5

STfrc $=$ the maximum fraction any given parameter can change between its lower and upper limits. A typical value is 0.25 
Frcsec $=$ determines Levenberg-Marquardt type value to constrain step length of the search vector and direction of search if the BFGS updates fail. Values of FRCSEC $>1$ cause the search vector to be short and oriented along a steepest-descent path. Values of FRCSEC $<0.01$ generally produce an unconstrained Gauss-Newton solution.

Rkmin $=$ is the threshold below which a diagonal value is treated as zero. For many problems, RKMIN should be greater than 0.001 and a value of 0.01 is more honest.

Cfi $=$ is the perturbation factor or influence coefficient for calculating gradients. Typically CFI $=0.01$ and should not be less than 0.001 because of rounding errors that occur while updating MODFLOW input files.

LIMIT:Choice $\quad=$ is a switch (REMove/Keep) that determines if parameter estimation will be attempted during successive iterations after a parameter is constrained by either its lower or upper limit. MODOPTIM defaults to keep attempting to estimate all parameters with every iteration.

DATA GROUP 4 - DEFINITION OF PARAMETER-WEIGHT MATRICES

OPTIONAL: If a weight matrix header is not read, no parameter-weight matrices will be defined and estimated parameters cannot be modified by a parameter-weight matrix.

\title{
REPEAT THE FOLLOWING DATA IN SEQUENCE FOR EACH PARAMETER-WEIGHT MATRIX - Weight matrix entry is terminated if the key [WEIGHT] is not present on data line 4A.
}

\author{
4A. KEY:DATA \\ TYPE: \\ WEIGHT:wtag (NORMalize:Y/N) (TYPE:iwp) \\ (INPUT:Entry) \\ $1.50 \quad$ Alphanumeric header card \\ $\begin{aligned} \text { WEIGHT:wtag }= & \text { each parameter-weight matrix is identified by an alphanumeric tag. Only a capitalized } \\ & \text { version of the first } 4 \text { letters is retained for parameter-weight matrix identification. }\end{aligned}$ \\ NORMalize:Y/N = parameter-weight matrix is normalized by dividing matrix values by the standard deviation \\ of all values in the matrix, including values in inactive cells. \\ OPTIONAL -- A parameter-weight matrix is normalized by default. \\ TYPE:iwp $\quad=$ specifies if the parameter-weight matrix is spatial or temporal. If it is spatial, iwp indicates \\ the number of spatial dimensions and the spatial orientation of the matrix in an XYZ \\ coordinate system. The following keys are used to specify iwp. \\ $\mathrm{X}=$ a one-dimensional, NCOL matrix \\ $\mathrm{Y}=$ a one-dimensional, NROW matrix \\ $\mathrm{Z}=$ a one-dimensional, NLAY matrix \\ $X Y=a$ two-dimensional, NCOL by NROW matrix \\ $\mathrm{XZ}=$ a two-dimensional, NCOL by NLAY matrix \\ $\mathrm{YZ}=$ a two-dimensional, NROW by NLAY matrix \\ $X Y Z=a$ three-dimensional, NCOL by NROW by NLAY matrix \\ $\mathrm{SP}=$ a one-dimensional, NPER matrix \\ OPTIONAL -- XYZ is the default setting. \\ INPUT:Entry $\quad=$ First character of entry controls how a parameter-weight matrix will be defined by \\ MODOPTIM. The choices are: \\ Polarize $=$ a spatial parameter-weight matrix is constructed from an iso-value contour that is described \\ by a series of $<x, y>$ pairs. A temporal parameter-weight matrix is constructed by \\ interpolating the time at the end of each stress period to a schedule defined by the $<$ time,y $>$ \\ pairs (Typically, one might be defining a production schedule for wells). Data entry is \\ terminated with the <end $>$ flag. The polarized parameter-weight matrix is filled with \\ positive distances from the iso-value contour on one side and negative values on the other \\ side.
}


$\underline{\text { Absolute }}=$ a parameter-weight matrix is constructed with only positive distances from the iso-value contour. Data entry is the same as for the Polarize option.

Direct $=$ read in a parameter-weight matrix of the dimensions specified in iwp directly.

OPTIONAL -- Direct entry is the default setting.

4B. DATA Weight

TYPE: $\quad$ Matrix reader

Weight $=$ parameter-weight matrix is read directly or as $\langle\mathrm{x}, \mathrm{y}\rangle$ pairs that define either an iso-value line or a temporal schedule (Data entry is defined by the options selected on line 4A). Data entry to the matrix reader is terminated with an '<end >' flag on the line that follows the last value read.

DATA GROUP 5 - DEFINITION OF OPTIMIZATION PARAMETERS

REPEAT THE FOLLOWING DATA IN SEQUENCE FOR EACH PARAMETER-Parameter entry is terminated if the key [PARA] is not present on data line $5 \mathrm{~A}$ or $<$ end $>$ is detected.

5A. KEY:DATA PARAMeter:ptag TAG:ptype (STATUS:istatus) TYPE:pinfo

TYPE: $\quad$ Alphanumeric header card

PARAMeter:ptag = An arbitrary and unique 4 character descriptor assigned to each parameter. Parameters are tracked and reported with this variable.

TAG:ptype $\quad=$ identifies which variables in MODFLOW the parameter ptag will modify. The MODFLOW variables are identified by the corresponding variable pname in the MODFLOW files. The identifiers ptype and pname are user-defined.

STATUS:istatus $\quad=$ First character is the key that determines how a parameter will be defined by MODOPTIM. The choices are:

$\underline{\text { Active }}=$ parameter is modified and estimated.

$\underline{\text { Modify }}=$ parameter is modified but is NOT estimated.

Inactive $=$ parameter definition is ignored

OPTIONAL -- Active is the default setting.

TYPE:pinfo = defines how a parameter modifies MODFLOW input and has 4 components that are composed of the first letter of the choices listed. For example, input of TYPE:Parallel,None,Exp,No will be stored in pinfo as 'PNEN'.

Table B.5A Options that can be specified for the PINFO variable.

\begin{tabular}{cccc}
\hline Modifier type & Zero Limits & Operator & Scaling \\
\hline Parallel & Lower & Exponential & $\underline{\text { Scale }}$ \\
Series & $\underline{\text { Upper }}$ & $\underline{\text { Multiplicative }}$ & $\underline{\text { No scaling }}$ \\
& $\underline{\text { None }}$ & $\underline{\text { Additive }}$ &
\end{tabular}

5B. KEY:DATA

(TAG:iglact)

TYPE:

LOWer:down $\quad=$ lower limit of parameter estimate

INItial:par $\quad=$ initial parameter estimate

UPper:upper $\quad=$ upper limit of parameter estimate
LOWer:down

Alphanumeric data input card
INItial:par UPper:upper 
TAG:iglact $\quad=$ an alphanumeric tag that identifies a weight matrix defined in the data group 4 input. If iglact is not defined, a uniform weight of one will be used.

5C. DATA

TYPE: parb *(igbact) [igbsp]

Alphanumeric data input card

parb $=$ a global multiplier that modifies the parameter across the zones specified by igbact and the stress periods specified by igbsp. This multiplier can be used to change units and does not change during parameter estimation.

igbact $=$ the zones to be modified by the parameter. Zones can be assigned individually or as groups. If the areas of the model domain are assigned integer identifiers, zones can be specified by zones =layer $\mathrm{x}$ step+area where step is a value of 10,100, or 1,000 and is larger than the greatest area identifier. For example,

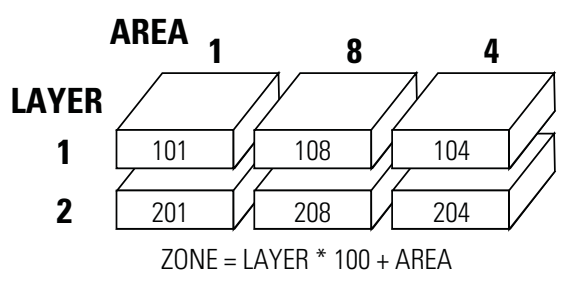

$(101,108,201)$ specifies the shaded zones:

\begin{tabular}{|l|l|l|}
\hline 101 & 108 & 104 \\
\hline 201 & 208 & 204 \\
\hline
\end{tabular}

$(200,104)$ specifies the shaded zones:

\begin{tabular}{|l|l|l|}
\hline 101 & 108 & 104 \\
\hline 201 & 208 & 204 \\
\hline
\end{tabular}

igbsp $=$ the stress periods during which the parameter will be modified. Stress periods are identified individually $[1,3,4,5]$ and as ranges [2 to 8]. A 'TO' placed between two stress periods denotes that MODFLOW input in all stress periods between the two limits will be modified by this parameter. For example, [2 to 5] specifies the MODFLOW input in stress periods 2, 3, 4, and 5 will be modified by the parameter.

\section{C cards can be repeated up to $m g b$ times, where $m g b$ is the maximum number of global multipliers as defined in the compiled program.}

Sample input of an element of GROUP 5 data:

\#

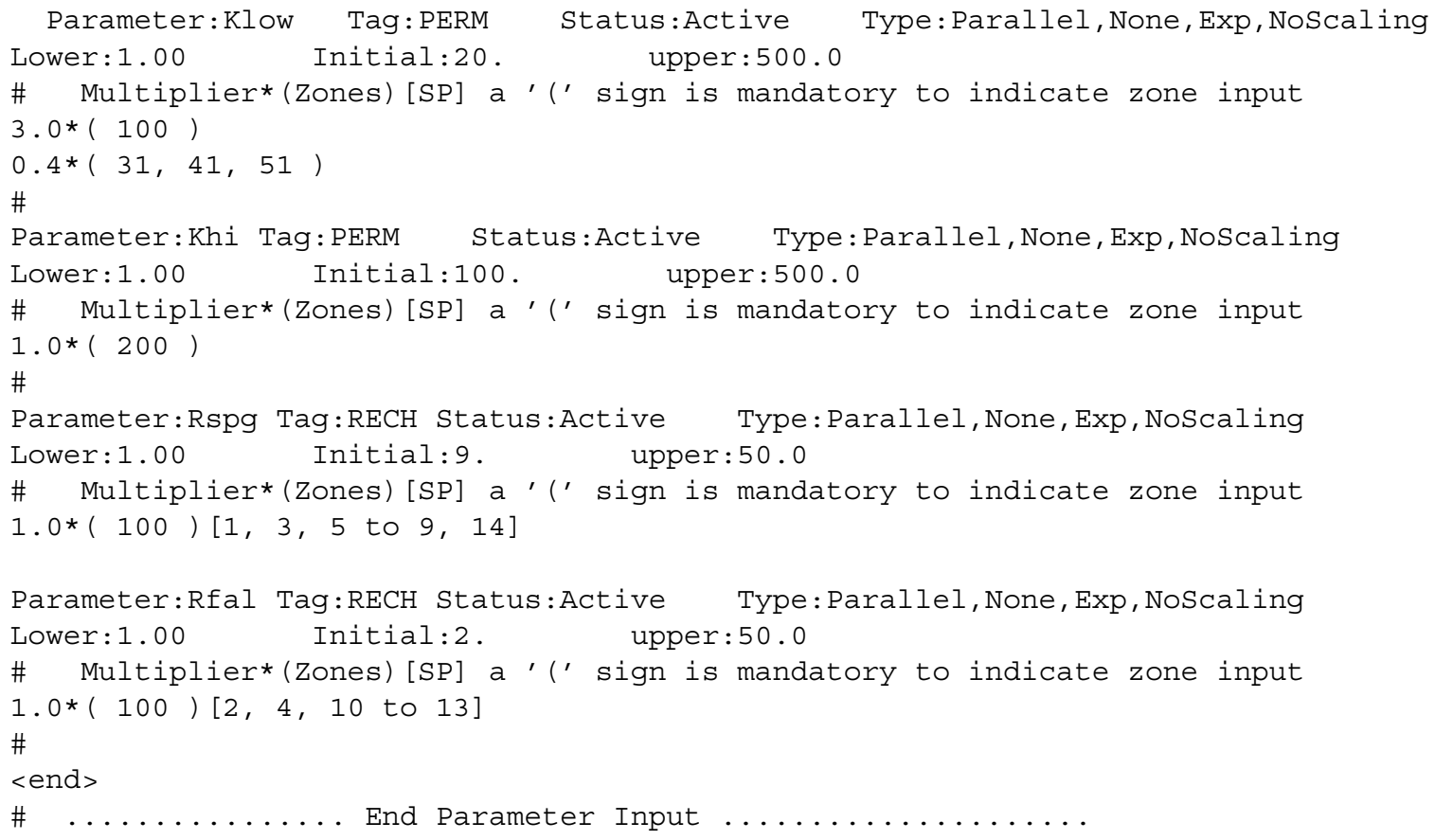


DATA GROUP 6 - GENERAL OBSERVATION MODIFIERS

OPTIONAL: All input before reading the observations have default values.

6. KEY:DATA The following observation modifiers can be entered in any order using as many lines that the user needs.

TYPE: $\quad$ Alphanumeric data input cards

START:timref $\quad=$ Identifies time 0 of simulation in time units of data input. Default is 0 .

SCALE:timscl $=$ Scales time units of data input to time units that are used within the MODFLOW simulation. Default is 1 .

PURPOSE: Allows user to modify observation times in data input by: $t_{M O D F L O W}=\left(t_{I N P U T}\right.$-time ref $)$ timscl. This option has been used to facilitate analyzing aquifer-test data.

OFFSET:wlref $\quad=$ Shifts datum of water levels in units of data input. Default is 0 .

MULT:wlmlt $\quad=$ Scales water-level units of data input to units that are used within the MODFLOW simulation. Default is 1.

PURPOSE: Allows user to modify observation values in data input by: $W L_{M O D F L O W}=\left(W L_{I N P U T}-\right.$ wlref $) w l m l t$. This option has been used to facilitate analyzing aquifer-test data.

INTIME $\quad=$ if either the fragment 'SS' or 'NO TIME' are found before the first observation site is read from the MODOPTIM input file, simulated water levels and flow rates will not be interpolated in time to the observation time. Default is to interpolate simulated values in time.

PURPOSE: Temporal interpolation is not appropriate if a steady-state or series of steady-state flow systems are being simulated.

ADJUST-WEIGHT $=$ a flag that normalizes weights for each observation type to the first observation type by standard deviation of each measurement type. Default is to leave weights unadjusted.

PURPOSE: Provides an initial estimate for scaling different observation types to sensitivities of similar magnitude.

FLOW:qtest $\quad=$ specifies the descriptor and number of characters that will be used to identify flow-rate observations. The text string specified in qtest is compared to otype which is defined on card

7A. If qtest is not specified, the default descriptor is 'FLOW' for all flow-rate comparisons.

PURPOSE: Allows for multiple flow-rate observation types to be classified.

MULT-Q:qmlt $=$ Scales flow units of data input to units that are used within the MODFLOW simulation. Default is 1 .

QUALITY:qwtest $=$ specifies the descriptor and number of characters that will be used to identify water-quality observations. The text string specified in qwtest is compared to otype which is defined on card 7A. If qtest is not specified, the default descriptor is 'CONC' for all water-quality comparisons.

PURPOSE: Allows for multiple water-quality observation types to be classified.

MULT-QW:qwmlt = Scales water-quality units of data input to units that are used within the MODFLOW simulation. Default is 1 .

COST:liftst $\quad=$ specifies the descriptor and number of characters that will be used to identify pumping-lift cost observations. The text string specified in liftst is compared to otype which is defined on card 7A. If liftst is not specified, the default descriptor is 'LIFT' for all pumping-lift cost comparisons.

PURPOSE: Allows for multiple pumping-lift cost observation types to be classified.

MULT-LIFT:cstmlt $=$ Scales lift units of data input to units that are used within the MODFLOW simulation. Default is 1 . 
OUTPUT:wminoc $=$ minimum weight an observation can have and still be printed to the output file of residuals. Default value of wminoc $=0$.

BLANK $=$ a flag that indicates blank lines should be printed between sites in the output file of residuals. Default is no blank lines.

DATA GROUP 7 - OBSERVATIONS

NOTE: The sequence of water-level, flow-rate, water-quality, pumping-lift cost, or subsidence observation entries are arbitrary. The user can enter flow-rate and water-level observations in whatever fashion the user deems reasonable. All observations will be normalized to the first observation type.

\title{
REPEAT THE FOLLOWING SEQUENCE FOR EACH OBSERVATION SITE -
}

\author{
7A. KEY:DATA OTYPE(:flowkey) (Z-INTERpolation:Y/N) (ABSOLute) LOCKEY:coordinate \\ SITE:well \\ TYPE: $\quad$ Alphanumeric header card
}

OTYPE a 4-character descriptor that identifies the values to be compared as either water-level, flow-rate, waterquality, or pumping-lift cost observations. Otype is set to the first 4 non-blank characters read from card 7A. For flow-rate, water-quality, or pumping-lift cost observations, otype must begin with the text string specified in qtest, qwtest, or liftst, respectively. If otype does not begin with the descriptor specified for qtest, qwtest, or liftst the observations from that site are assumed to be water levels.

(:flowkey) character strings that identify the flow components to be compared. This is only needed for flow-rate observations. Flowkey is set to MNW for water-quality and pumping-lift cost observations. The flowkeys and flow components are listed below:

Table B.7A List of flow components from MODFLOW modules that can be compared to specified discharge rates.

\begin{tabular}{ll}
\hline \multicolumn{1}{c}{ FLOWKEY } & \multicolumn{1}{c}{ Flow component from MODFLOW module } \\
\hline STORage & Storage from SBCF5S \\
WEL or MNW & Specified discharge from WEL5BD and MNW1BD \\
DRAIn & Head dependent discharge from DRN5BD \\
RIVEr or STREam & Head dependent discharge from RIV5BD and STR5BD \\
ET & Evapotranspiration from EVT5BD \\
CHD or SPECified head & Specified heads from SBCF5F \\
GHB & General head boundary from GHB1BD \\
RECHarge & Specified recharge from RCH5BD \\
\hline
\end{tabular}

(Z-INTERpolation:Y/N)simulated water levels can either be or not be interpolated between layers.

OPTIONAL -- Default condition is to NOT interpolate water levels between layers.

(ABSOLute) simulated flow rates for a site are the summation of the absolute value of all flow terms. The ABSOLute switch allows a penalty to be imposed on the overall magnitude of stresses if the observation sums both sink and source terms.

OPTIONAL -- The default condition is to sum positive and negative flow rates. 
(LOCKEY:)

identifies discrete locations for water-level, water-quality, and pumping-lift cost observations or zones that define a volume for summing flow-rate and pumping-lift cost observations and flow-rate-weighted averaging of water quality observations. For discrete observations, three variables are read according to the table below. Simulated water levels are interpolated to the measured water level location. Discrete water-quality and pumpinglift cost observations are compared at the node with a pumping well. Observations that are summed or averaged over a volume are defined by as many as eight zones that correspond to zone assignments in the IOBFLX array.

Table B.7B List of LOCKEY variables for identifying the method of specifying observation locations.

\begin{tabular}{|c|c|c|c|}
\hline LOCKEY: & Coordinate & OTYPE & $\begin{array}{l}\text { Number of } \\
\text { variables }\end{array}$ \\
\hline XYZ: & Cart-X, Cart-Y, Cart-Z & \multirow{8}{*}{$\begin{array}{c}\text { Water Level, } \\
\text { Water Quality, } \\
\text { Subsidence, } \\
\text { and Pumping-Lift Cost }\end{array}$} & 3 \\
\hline XYK: & Cart-X, Cart-Y, Layer & & 3 \\
\hline XJZ: & Cart-X, Row, Cart-Z & & 3 \\
\hline XJK: & Cart-X, Row, Layer & & 3 \\
\hline IYZ: & Column, Cart-Y, Cart-Z & & 3 \\
\hline IYK: & Column, Cart-Y, Layer & & 3 \\
\hline IJZ: & Column, Row, Cart-Z & & 3 \\
\hline IJK: & Column, Row, Layer & & 3 \\
\hline ZONE: & $\begin{array}{l}\text { Zone identifiers specified in } \\
\text { the IOBFLX array }\end{array}$ & $\begin{array}{c}\text { Flow Rate, Water } \\
\text { Quality, and Pumping- } \\
\text { Lift Cost }\end{array}$ & $1-8$ \\
\hline
\end{tabular}

SITE:well $\quad=$ an alphanumeric descriptor that is used to identify an observation site.
7B. KEY:DATA
wellbore STORage Kxy
Lscreen $\quad \mathrm{Rw}$
TAG:pname

TYPE:

Alphanumeric header card

OPTIONAL: This card is optional and is only read if it immediately follows card 7A. Cards 7B and 7C are mutually exclusive.

wellbore STORage = indicates wellbore storage effects in the observation wells will be simulated and the required variables will be read from this card.

Kxy $=$ horizontal hydraulic conductivity of the material around the wellbore.

Lscreen $=$ the length of the well screen.

rw $\quad=$ the radius of the well casing

The three variables $(K x y$, Lscreen, $r w)$ are reduced to one variable $\beta=\frac{{ }_{S C R E E N} K_{X Y}}{2 \ln \left(\frac{L_{S C R E E N}}{r_{w}}\right) r_{w}^{2}}$ which is

used in the equation

$h w_{m}=\frac{\beta \Delta t\left(h_{m}+h_{m-1}-h w_{m-1}\right)+h w_{m-1}}{1+\beta \Delta t}$ to estimate the water level in the observation well 
TAG:pname $\quad=$ is the name of the MODFLOW variable tracked if $\beta$ is estimated independently or if $\beta$ is coestimated with the hydraulic-conductivity distribution that surrounds the well screen.

7C. KEY:DATA

TYPE:
PUMPing well losses $\quad \mathrm{T}$

Alphanumeric data input card
Rw

TAG:pname

OPTIONAL: This card is optional and is only read if it immediately follows card 7A. Cards 7B and 7C are mutually exclusive. Use of this option forces the observation to the nearest node which is presumably being stressed by a well.

PUMPing well losses= indicates head losses between node and pumping well will be simulated and the required variables will be specified.

$\mathrm{T}=$ lateral transmissivity of the material around the wellbore.

$\mathrm{Rw}=$ the radius of the well casing

The two variables $(\mathrm{T}, \mathrm{rw})$ are reduced to one variable $\beta=\frac{2 \pi T}{\ln \left(r_{o} / r_{w}\right)}$ where $r_{o}=0.14 \sqrt{\Delta x^{2}+\Delta y^{2}}, \Delta x$ is the width of the column, and $\Delta y$ is the width of the row. The equivalent radius of the pumping cell $\left(r_{o}\right)$ was defined by Peaceman (1983). The difference in head between the well node and the wellbore is estimated by $\Delta h=Q / \beta$ where $Q$ is the rate of water withdrawal or injection.

TAG:pname $\quad=$ is the name of the MODFLOW variable tracked if $\beta$ is estimated independently or if $\beta$ is coestimated with the hydraulic conductivity distribution that surrounds the well screen.

7D. DATA

TYPE:

time

observation

$(<,>)$

(-weight)

(+weight)

(Default)

(Reference)

DXyz:well2, time2 $=$

DT $=$ the observation read from the previous card is the reference observation. If DT is specified for the first observation at a site, no comparison will be made and the weight is set to zero.

NO comparison sets -weight and +weight to 0.00 for sites that are reference observations.

OFFSET sets this observation as the reference observation for all subsequent observations until a new site is specified. Simulated and measured observations are both set to zero for this entry which does not affect the objective function. 
DATA GROUP 8 - DEFINITION OF SUMMED AND FLOW-RATE AVERAGED OBSERVATION ZONES

OPTIONAL: If flow zones are not defined in data group 8, the flow zones in the IOBFLX array are set equal to the parameter zones in the IZCUBE array.

\section{A. KEY:DATA FLUXZONES Dimension:size (BY:step)}

TYPE:

Alphanumeric header card

FLUXZONES $=$ the flag that signals data group 8 will be read.

Dimension:size $=$ denotes whether the matrix read to define the flow zones are 1-, 2-, or 3-dimensional, and the orientation of the matrix in an XYZ coordinate system as denoted by the type flags:

GLOBAL $=$ a single value

$$
\mathrm{Z}=\text { a one-dimensional, NLAY matrix }
$$

$X Y=a$ two-dimensional, NCOL by NROW matrix

$X Y Z=$ a three-dimensional, NCOL by NROW by NLAY matrix

OPTIONAL -- XYZ is the default setting.

(BY:step) = increases zone values by zones $=$ layer $\mathrm{x}$ step $+i z n$ where step is typically a value of 10 , 100 , or 1,000 and is larger than the greatest area identifier. allows zones to be modified by the parameter.

8B. DATA iobflx

TYPE: $\quad$ Matrix reader

iobflx = zones for summing flow-rate and lift-cost observations and averaging flow-rate weighted water quality observations. Data entry to the matrix reader is terminated with an '<end>' flag on the line that follows the last value read.

Sample input with elements of GROUP 7 and 8 data: 


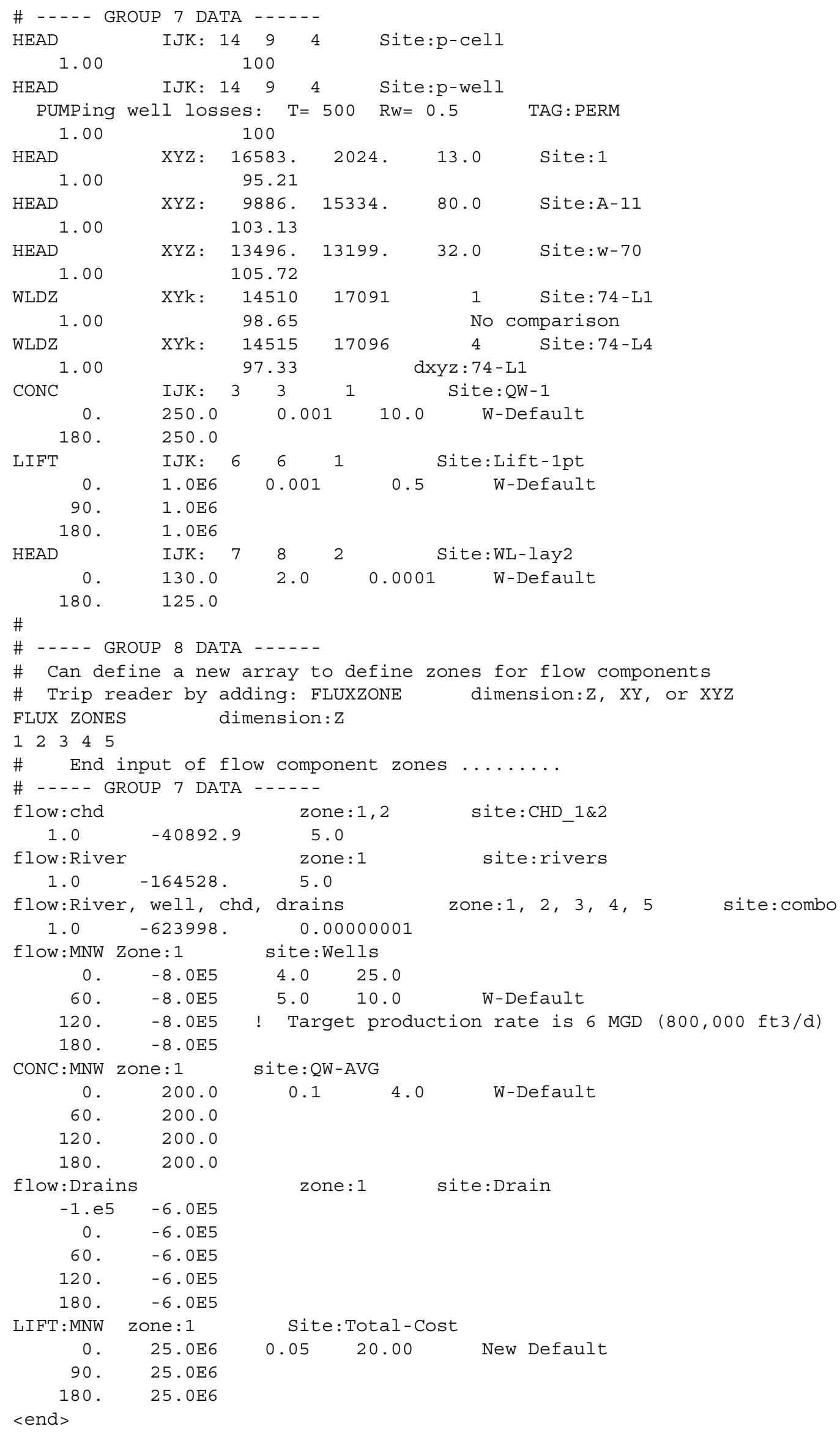




\section{Appendix C Input for composite MODFLOW/MODOPTIM file with parameters to be estimated}

DATA GROUP 1- General data for building MODFLOW / BAS file

1A. DATA txt

TYPE: $\quad$ Alphanumeric header card

txt $=$ is the model description and is read on two cards

1B. KEY:DATA File with 3D grid definition: filename

TYPE: $\quad$ Alphanumeric data input card

filename $=$ is the name of the file with the column, row, and layer dimensions, the column and row widths, a reference location, the thickness of the model cells, and the elevation of the model nodes.

1C. DATA itmuni

TYPE: $\quad$ integer

itmuni $=$ specifies the time unit in MODFLOW. ITMUNI can range from 0 to 5 to specify time as undefined, seconds, minutes, hours, days, or years, respectively. If ITMUNI is not specified or is not in the range of 0 to 5 , it is set to 4 .

DATA GROUP 2 - Specification of stress period and time step lengths

2A. DATA perlen nstp tsmult

TYPE: $\quad$ Matrix reader

perlen $=$ stress period length

nstp $\quad=$ number of time steps in a stress period

tsmult = time step multiplier as defined by McDonald and Harbaugh (1988)

NOTE: variables are read until an '<end>' flag is encountered. The number of variables read must be divisible by three or MODOPTIM will halt and report the error.

DATA GROUP 3 - SPECIFY MODFLOW FILES AND UNIT NUMBERS

3A. KEY:DATA

FILE:filename

MODKEY:unit

TYPE:

Alphanumeric data input card

FILE:filename $\quad=$ name of MODFLOW data input file to be opened. 
MODKEY:unit $\quad=$ the unit number associated with the filename. MODKEY identifies the MODFLOW module that will read data from the file and the IUNIT location assigned in the BAS package. MODFLOW data input files that will be built by MODOPTIM must be assigned a unit number between 10 and 39, inclusive. Unit numbers 40 through 69, inclusive, are reserved by MODOPTIM. The recognized MODKEY and MODFLOW modules are listed below:

Table C.3A List of MODKEY flags that identify a MODFLOW module.

\begin{tabular}{|c|c|c|}
\hline MODKEY & MODFLOW module & IUNIT \\
\hline $\mathrm{BCF}$ & Block Centered Flow & 1 \\
\hline WEL & Wells & 2 \\
\hline DRN & Drains & 3 \\
\hline RIV & River & 4 \\
\hline EVT & Evapotranspiration & 5 \\
\hline TLK & Transient Leakage & 6 \\
\hline GHB & General head boundary & 7 \\
\hline $\mathrm{RCH}$ & Recharge & 8 \\
\hline SIP & Strongly Implicit Procedure & 9 \\
\hline DE4 & Direct Solver & 10 \\
\hline SOR & Slice Successive Over Relaxation & 11 \\
\hline $\mathrm{OC}$ & Output Control & 12 \\
\hline PCG2 & Pre-Conditioned Gradient & 13 \\
\hline GFD & General Finite Difference & 14 \\
\hline HFB & Horizontal Flow Barrier & 16 \\
\hline RES & Reservoir & 17 \\
\hline STR & Stream Routing & 18 \\
\hline IBS & Interbed Storage & 19 \\
\hline CHD & Time-Dependent Specified head & 20 \\
\hline FHB & Flow and Head Boundary & 21 \\
\hline VAR1 & Time-Dependent BCF & 22 \\
\hline MNW & Multi-Node, drawdown-limited wells & 23 \\
\hline
\end{tabular}

NOTE: Files are read, assigned unit numbers, and opened until an '<end>' statement is encountered.

3B. KEY:DATA

TYPE:
FILE:filename Alphanumeric data input card

FILE:filename $\quad=$ name of unformatted MODFLOW data output file to be opened.

$<$ unit $>\quad=$ the unit number associated with the filename.

NOTE: Files are read, assigned unit numbers, and opened until an '<end>' statement is encountered.

Sample input of GROUP 3 data: 


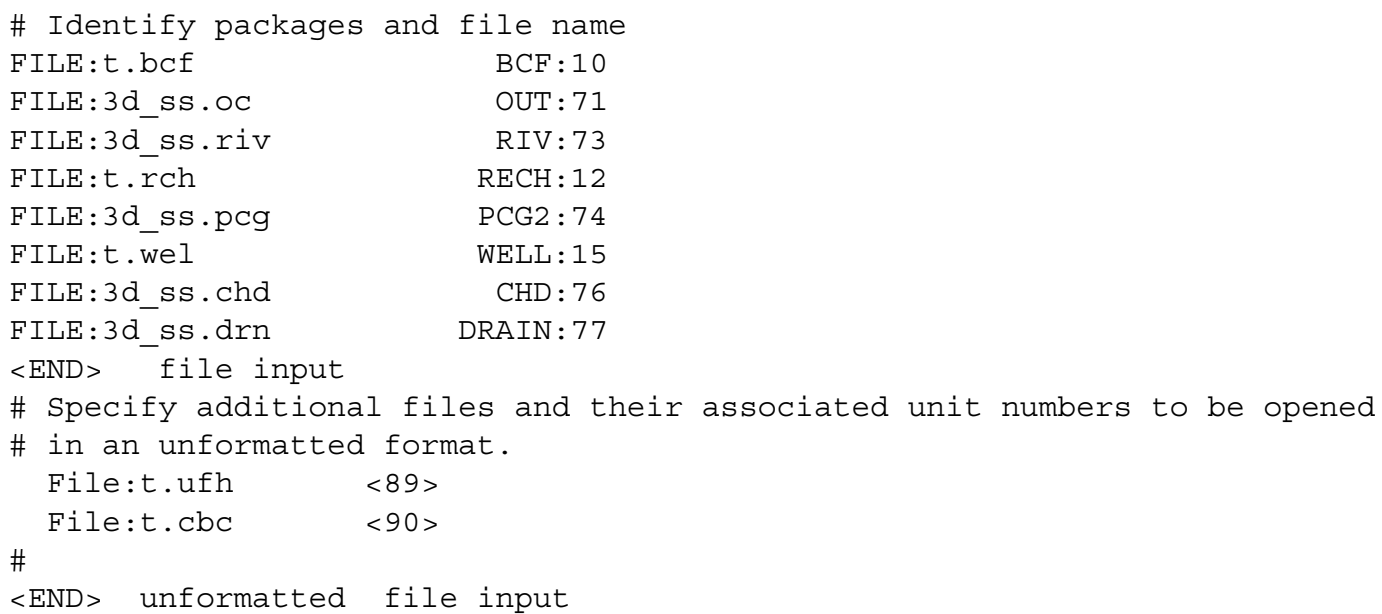

DATA GROUP 4 - Specification of MODFLOW variables IAPART and ISTRT

$\begin{array}{lll}\text { 4A. DATA } & \text { iapart } & \text { istrt } \\ \text { TYPE: } & \text { integer } & \text { integer }\end{array}$

iapart = indicates whether array BUFF is separate from array RHS. If iapart $=0$, arrays BUFF and RHS occupy the same space (McDonald and Harbaugh, 1988)

istrt $=$ indicates whether starting heads are to be saved. If $i s t r t=0$, starting heads are not saved. (McDonald and Harbaugh, 1988)

NOTE: If these values are not on this card, iapart defaults to 0 and istrt defaults to 1 .

DATA GROUP 5 - PARAMETER ZONES / IBOUND ARRAYS

Read either cards 5A and 5B or cards 5C, 5D, and 5E. Direct entry and polygon entry are mutually exclusive of one another.

If zones are defined directly as integer arrays, read cards 5A and 5B once-

\section{A. KEY:DATA DIRECT (BY:step)}

TYPE: $\quad$ Alphanumeric header card

DIRECT = specifies that either 1, nlay, ncol by nrow, or ncol by nrow by nlay values be read and that the IBOUND and IZCUBE arrays will have 1 value, uniform values in each layer, unique areal zones, or unique zones throughout the model volume.

BY:step $\quad=$ increases zone values by zones $=$ layer $\mathrm{x}$ step $+i z n$ where step is typically a value of 10 , 100 , or 1,000 and is larger than the greatest area identifier. This is valid only for a $n c o l$ by nrow array of integers.

5B. DATA izcube

TYPE: Matrix reader

izcube $\quad=$ is an array of zone values. Data entry to the matrix reader is terminated with an ' $<$ end $>$ ' flag on the line that follows the last value read. 
If zones are defined by polygons, card $5 \mathrm{C}$ can be used to reassign the default zone number.

5C. KEY:DATA

TYPE:

GLOBal:igbzn = GLOB is the key that identifies a new default zone identifier. Igbzn is the default zone identifier that was specified by the user. If card 5C is not read, the default zone identifier is 0 .

Each polygon is specified with repeated sets of cards $5 \mathrm{D}$ and $5 \mathrm{E}-$

5D. KEY:DATA ZONE:izn LAYER:layers $\quad$ (BY:step)

TYPE:

ZONE:izn $\quad=$ ZONE: is the key that identifies the zone will be an area specified by a polygon. Izn specifies an element of the zone identifier used in ibound and izcube. If BY:step is not used, $i z n$ is equal to the zone number. Positive values of $i z n$ are used to assign zone numbers to nodes inside the polygon while negative values of $i z n$ indicate that zone numbers will be assigned to nodes outside the polygon with the absolute value of $i z n$.

LAYER:layers $\quad=$ specifies layers where polygon will define zone. Negative value for layer serves to flag specified head in ibound.

BY:step $\quad=$ increases zone values by zones $=$ layer $\mathrm{x}$ step $+i z n$ where step is typically a value of 10,100, or 1,000 and is larger than the greatest area identifier.

EXAMPLE: Usage of polygons to delineate zones is illustrated below:

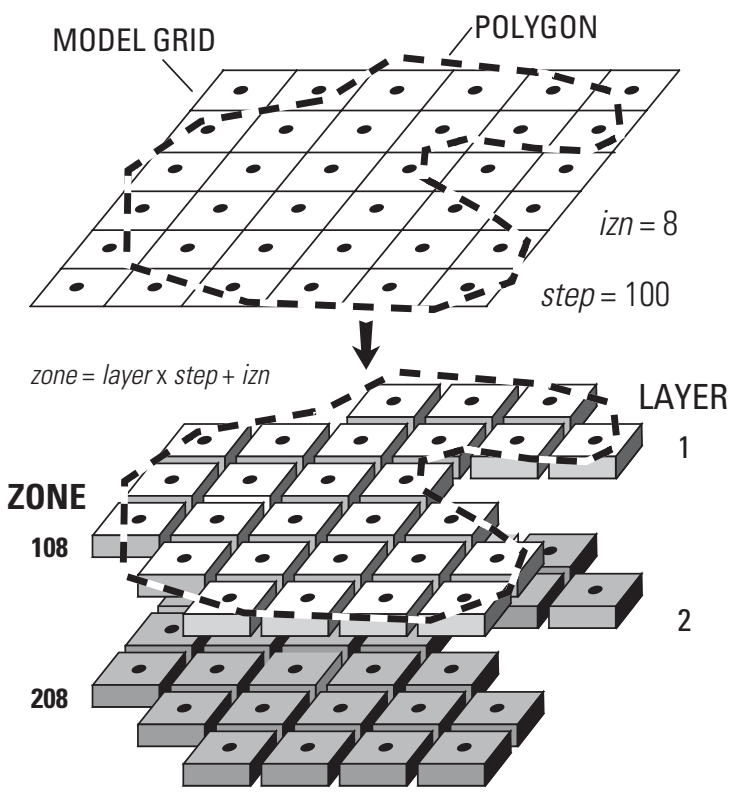

5E. DATA

izcube

TYPE:

Matrix reader 
izcube

$=$ is an array of $\langle\mathrm{x}, \mathrm{y}\rangle$ pairs that define a polygon. Data entry to the matrix reader is terminated with an '<end>' flag on the line that follows the last value read.

NOTE: Zone values also can be modified on a cell-by-cell basis if the WEL1, WEL2, CHD, DRN, GHB, RIV, STR, or VAR packages are constructed by MODOPTIM.

DATA GROUP 6 - INACTIVE CELL AND STARTING HEAD VALUES

6A. DATA

hnoflo

TYPE:

real

hnoflo $=$ head value assigned to inactive cells. Default value of hnoflo is 1234567.

6B. KEY:DATA

TYPE:

(UNIT:iohed) = specifies unit number for file with starting head values. The default assignment of iohed is 39. If iohed is less than 0 , it is assumed the starting heads are stored in an unformatted file.

(GLOBAL:globe) = is a global starting head value that is assigned to all nodes if a file of starting heads does not already exist. This is an OPTIONAL assignment.

FILE:name $\quad=$ is the name of the file with the starting head values to be read by MODFLOW.

\section{General description of DATA GROUPS 7, 8, 9 and 10}

Data groups 7, 8, 9, and 10 describe the construction of MODFLOW files with parameters to be estimated. Much of the input for data groups 7, 8, 9, and 10 is similar to the input for a MODFLOW simulation without optimization. The MODFLOW files are constructed from the composite MODFLOW/MODOPTIM file to facilitate the placement of name tags (variable pname) that identify the hydraulic characteristics and stresses to be modified. The hydraulic characteristics and stresses in MODFLOW that can be estimated by MODOPTIM are read from files as ncol by nrow arrays (storage, transmissivity, vertical leakance, recharge, evapotranspiration) or as cell-by-cell lists (general head boundaries, rivers, specified heads, drains, wells).

DATA GROUP 7 - Header for Packages with Hydraulic Properties to be Estimated

7A. KEY:DATA

TYPE:

PACKAGE $=$ MODKEY The MODFLOW module to be constructed is identified by the MODKEY variable which must equal one of the identifiers specified in table C.3A.

7B. DATA

TYPE:
PACKAGE $=$ MODKEY

Alphanumeric header card text

Alphanumeric card

text

Data sets that are needed in MODFLOW but are not needed by MODOPTIM. These cards are read and echoed into the MODFLOW packages. These cards are read and echoed until a pname tag or an <end> flag is read.

NOTE: Cards are read and entered in the package constructed until an <end $>$ flag is encountered. 
DATA GROUP 8 - Additional Data Needed to Construct a BCF Package

These cards are read if $M O D K E Y$ is equal to $\mathrm{BCF}$.

8A. KEY:DATA Trans CBC:unit

TYPE: $\quad$ Alphanumeric data input cards

Trans $=$ a transient BCF package will be constructed if the fragment 'TRAN' is detected. Otherwise, the default assumption is that a steady-state model is being simulated.

CBC:unit= specifies unit number for cell-by-cell output from BCF package to unformatted file.

8B. DATA Laycon

TYPE: $\quad$ integer

Laycon $=$ specifies layer type and data sets to be read. LAYCON values: 0 - confined, 1 - unconfined, 2 confined/unconfined with transmissivity held constant, and 3 - fully convertible between confined/unconfined (McDonald and Harbaugh, 1988) Any unspecified layer will be assumed to be confined (laycon $=0$ ).

8C. DATA Trpy

TYPE: $\quad$ real

Trpy $=$ specifies an anisotropy factor for each layer in terms of a column to row ratio $\left(\operatorname{Trpy}=K_{Y} / K_{X}\right)$ (McDonald and Harbaugh, 1988). Any unspecified values of TRPY will be assumed to be equal to 1.

Card 8D is optional and terminates further entry for the BCF package.

8D. KEY:DATA AUTO (LUMP)

TYPE: $\quad$ Alphanumeric data input cards

AUTO = automatically creates a BCF package from the vertical discretization that was specified in the GR3 file if the fragment 'AUTO' is detected. Transmissivity arrays are assigned a pname of PERM. Vertical leakance arrays are assigned a pname of VCON. Storage coefficient arrays are created and assigned a pname of STOR only if the simulation is transient.

$($ LUMP $)=$ assigns a pname of PERM to both transmissivity and vertical leakance arrays so they can be estimated as a single parameter. This is an OPTIONAL assignment.

NOTE: The widths of the columns and rows are not read because they were specified previously on card C.1B.

DATA GROUP 9 - Identifying estimated hydraulic properties or stresses that are stored in a ncol by nrow array

9A. KEY:DATA

TYPE:

TAG:pname
TAG:pname Layer:laypro

Alphanumeric data input cards
(SP:itime)

$=$ is the user defined, 4-character tag that corresponds to the ptype variable defined on card B.5A in the OPT input file. Pname denotes if the property to be varied is a hydraulic conductivity, recharge, vertical leakance, or any other array of a hydraulic property or stress. 
Layer:laypro $\quad=$ identifies the model layer for comparing IZONE numbers to the zone numbers that are specified in the igbact array before an optimization parameter can modify the MODFLOW array.

Layer numbers can be greater than the number of model layers.

SP:itime (OPTIONAL) identifies the stress period that corresponds to the stress periods specified in the igbsp array before an optimization parameter can modify the MODFLOW array. If itime is not specified, itime is set equal to 1 .

9B. DATA Locat Cnstnt Fmtin Iprn

TYPE: $\quad$ Alphanumeric data input card

Locat is a flag. If Locat $=0$, all elements are set equal to Cnstnt and card 9C is not read (McDonald and Harbaugh, 1988). If Locat $>0, n c o l$ by nrow values are read. Locat is not used as a unit number. The unit number written to the constructed MODFLOW package is equivalent to the unit number assigned in card 3A.

Cnstnt = Constant to which all array values are set if Locat is equal to zero or by which all array values are multiplied if Locat is not equal to zero (McDonald and Harbaugh, 1988).

Fmtin $=$ Format under which arrays are written and read from the constructed MODFLOW file. A format that maintains at least five significant figures should be used.

Iprn $\quad=$ MODFLOW code for format to be used when printing arrays (McDonald and Harbaugh, 1988).

9C. DATA work

TYPE: $\quad$ Real array

work = ncol by nrow values are read (free-format) into the temporary work array and are written in fixed format to the constructed MODFLOW package.

NOTE: Cards 9A and 9B are read for every array that contains hydraulic properties or stresses to be modified by optimization parameters.

If the RADIAL flag was set on card D.1A, all arrays to be estimated will be multiplied by $2 \pi$ times an array of the $\mathrm{X}$-coordinate of the columns.

DATA GROUP 10 - Identifying estimated hydraulic properties or stresses that are stored in cell-by-cell lists.

10A. KEY:DATA TAG:pname Field:Lf SP:itime

TYPE: $\quad$ Alphanumeric data input card

TAG:pname $\quad=$ is the user defined, 4-character tag that corresponds to the ptype variable defined on card B.5A in the OPT input file. Pname denotes if the property to be varied is a pumping rate, riverbed conductance, specified head, or any other cell-by-cell list of hydraulic properties or stresses.

Field:Lf $\quad=$ The presence of the Field flag instead of the Layer flag indicates that a cell-by-cell list follows. The variable $L f$ identifies the field of the cell-by-cell list input file that is to be modified. For example, flow rates that are specified in the well package are in the $4^{\text {th }}$ field and riverbed conductances that are specified in the river package are in the $5^{\text {th }}$ field.

SP:itime (OPTIONAL) identifies the stress period that corresponds to the stress periods specified in the igbsp array before an optimization parameter can modify the MODFLOW array. If itime is not specified, itime is set equal to 1 . 
10B. DATA

ITMP

TYPE:

Integer

ITMP is a flag. If ITMP $<=0$, cell-by-cell data from a previous stress period will be reused and input from item $10 \mathrm{C}$ will not be read. If ITMP $>0$, it is the number of records of cell-by-cell data that will be read for the current stress period.

10C. DATA

TYPE:

txt

(ZONE:mz)

(KOFF:koff)
txt
(ZONE:mz)
(KOFF:koff)

Alphanumeric data input card is a cell-by-cell data entry. Values are read using free-format to the $\mathrm{Lf}^{\text {th }}$ field specified. The first three values (Layer, row, and column indices) are written to a (I10) format and the remaining values to the $\mathrm{Lf}^{\text {th }}$ field specified are written to a (G10.4) format. Modifies non-zero zone assignments in izcube array to $m z$ for each entry. Assigns an offset of koff to cell-by-cell layer assignment which allows for cell-by-cell entries in multiple layers to be specified by a single layer.

NOTE: Card 10A is read once for each cell-by-cell list MODFLOW package. It is assumed that only one field will be estimated in any package and that the same field will be estimated for all stress periods.

Input item 10C consists of one card for each cell-by-cell property specified. If ITMP is less than or equal to zero, item $10 \mathrm{C}$ is not read. 


\section{Appendix D Input for grid discretization file}

DATA GROUP 1 - Width of columns and reference for the X-coordinate
1A. DATA
ncol
(xref)
(ixref)
(MULT)
(RADIAL)

TYPE:

Alphanumeric header card

ncol is the number of model columns in the X-coordinate.

(xref) is the $\mathrm{X}$-coordinate at the node in model column ixref. Default reference is $\mathrm{X}=0$ at the left-edge of the model.

(ixref) is the column of the reference node that coincides with the X-coordinate (xref).

(MULT) is a flag that indicates the last value read in the $d x m$ array is the width of the model (the sum of $d x m$ values from 1 to $n c o l$ ). If the MULT flag is set, between 2 and $n c o l-1$ values will be read. The remaining columns are filled by a uniform multiplier which successively increases the widths of the following columns until the total model width is equal to the last value read in the $d x m$ array.

(RADIAL) is a flag that indicates the model will simulate radial flow. A radial model can have only one row or one layer. The reference of the $\mathrm{X}$-coordinate is set to the default setting of $\mathrm{X}=0$ at the left-edge of the model. All arrays that are estimated in the BCF package are multiplied by an array of $2 \pi$ times the Xcoordinate of the columns.
1B. DATA
dxm
TYPE:
Matrix reader

$\mathrm{dxm} \quad$ is the width of the model columns. Typically, one uniform value is read or an array of ncol values are read. If the MULT flag is set, between 2 and ncol-1 values will be read. (See MULT-flag explanation) Data entry to the matrix reader is terminated with an '<end>' flag on the line that follows the last value read.

DATA GROUP 2 - Width of rows and reference for the Y-coordinate

2A. DATA nrow $\quad$ (yref) (iyref) (MULT)

TYPE: $\quad$ Alphanumeric header card

nrow is the number of model rows in the Y-coordinate.

(yref) is the Y-coordinate at the node in model row iyref.

(iyref) is the row of the reference node that coincides with the Y-coordinate (yref).

(MULT) is a flag that indicates the last value read in the $d y m$ array is the height of the model (the sum of $d y m$ values from 1 to nrow). If the MULT flag is set, between 2 and nrow-1 values will be read. The remaining columns are filled by a uniform multiplier which successively increases the widths of the following rows until the total model width is equal to the last value read in the dym array.

2B. DATA dym

TYPE: $\quad$ Matrix reader

dym is the width of the model rows. Typically, one uniform value is read or an array of nrow values are read. If the MULT flag is set, between 2 and nrow-1 values will be read. (See MULT-flag explanation) Data entry to the matrix reader is terminated with an '<end>' flag on the line that follows the last value read. 
DATA GROUP 3 - Thickness of layers and elevation of nodes

Data groups 3, 4, and 5 are mutually exclusive methods of defining vertical discretization.

3A. DATA nlay (izpls) (q3dthk)

TYPE: $\quad$ Alphanumeric header card

nlay is the number of model layers in the Z-coordinate.

(izpls) is the number of layers in excess of model layers that will be specified for defining parameter zones.

(q3dthk) is the gap between layers that must be exceeded before water-level and subsidence observations are interpolated with a quasi-3D interpretation of vertical leakances.

3B. DATA dzm

TYPE: $\quad$ Matrix reader

dzm is a ncol by nrow by nlay array of cell thicknesses. Either 1, nlay, or ncol $\mathrm{x}$ nrow x nlay values are read by the matrix reader. A uniform thickness is assigned to all cells if 1 value is read and to each layer if nlay values are read. Otherwise each cell thickness is assigned individually if ncol x nrow x nlay values are read. If the number of values read is not equal to 1, nlay, or ncol x nrow x nlay, MODOPTIM will halt and the error will be reported. Data entry to the matrix reader is terminated with an '<end>' flag on the line that follows the last value read.

3C. DATA

TYPE:

$\mathrm{zc}$

Matrix reader

$\mathrm{zc}$

is a ncol by nrow by nlay array of model node elevations. Either 1, nlay, ncol x nrow, or ncol x nrow $\mathrm{x}$ nlay values are read by the matrix reader. If 1 or ncol $\mathrm{x}$ nrow values are read, the array read is used as the top of the uppermost layer and the elevation of each node is computed by assuming all layers are contiguous. If nlay values are read, a uniform elevation is assigned to all nodes within each layer. If $n c o l$ $\mathrm{x}$ nrow $\mathrm{x}$ nlay values are read, the elevation of each node is assigned individually. If the number of values read is not equal to 1, nlay, ncol $\mathrm{x}$ nrow, or ncol $\mathrm{x}$ nrow $\mathrm{x}$ nlay, MODOPTIM will halt and the error will be reported. Data entry to the matrix reader is terminated with an '<end>' flag on the line that follows the last value read.

DATA GROUP 4 - Contacts of layers
4A. DATA
nlay (izpls) CONTACT

TYPE:

Alphanumeric header card

nlay is the number of model layers in the Z-coordinate. Nlay can be specified as 0 and will be counted by MODOPTIM.

(izpls) is the number of layers in excess of model layers that will be specified for defining parameter zones.

CONTACT is a flag that indicates vertical discretization will be defined by $n l a y+l$ contacts. The top of layer $\mathrm{k}$ is coincident with the bottom of layer k-1 if vertical discretization is defined with contacts.

\section{Card $4 \mathrm{~B}$ is read nlay+1 times to define the thicknesses and elevations of all layers.}

4B. DATA work

TYPE: Matrix reader 
work $\quad$ is a ncol by nrow array. Either 1 or ncol $\mathrm{x}$ nrow values are read by the matrix reader. A uniform elevation is assigned to all cells if 1 value is read. Otherwise each elevation is assigned individually. If the number of values read is not equal to 1 or ncol $\mathrm{x}$ nrow, MODOPTIM will halt and the error will be reported. Data entry to the matrix reader is terminated with an '<end>' flag on the line that follows the last value read.

DATA GROUP 5 - Tops and Bottoms of layers

5A. DATA

nlay (izpls) (q3dthk)

TOPandBOTTOM

TYPE:

Alphanumeric header card

nlay is the number of model layers in the Z-coordinate. Nlay can be specified as 0 and will be counted by MODOPTIM.

(izpls) is the number of layers in excess of model layers that will be specified for defining parameter zones.

(q3dthk) is the gap between layers that must be exceeded before water-level and subsidence observations are interpolated with a quasi-3D interpretation of vertical leakances.

TOPandBOTTOM is a flag that indicates vertical discretization will be defined with a top and bottom surface for each layer.

Cards 5B and 5C are read nlay times to define the top and bottom elevations of all layers.

5B. DATA top

TYPE: $\quad$ Matrix reader

top is a ncol by nrow by nlay array of layer top elevations. Either 1 or ncol $\mathrm{x}$ nrow values are read by the matrix reader for each layer. A uniform top elevation is assigned to all cells in a layer if 1 value is read. Otherwise each top elevation is assigned individually. If the number of values read is not equal to 1 or ncol $\mathrm{x}$ nrow, MODOPTIM will halt and the error will be reported. Data entry to the matrix reader is terminated with an '<end>' flag on the line that follows the last value read.

5C. DATA bottom

TYPE: $\quad$ Matrix reader

bottom is a ncol by nrow by nlay array of layer bottom elevations. Either 1 or ncol $\mathrm{x}$ nrow values are read by the matrix reader for each layer. A uniform bottom elevation is assigned to all cells in a layer if 1 value is read. Otherwise each bottom elevation is assigned individually. If the number of values read is not equal to 1 or ncol x nrow, MODOPTIM will halt and the error will be reported. Data entry to the matrix reader is terminated with an '<end>' flag on the line that follows the last value read. 


\section{Appendix E Time-variant hydraulic-property package (VAR1)}

The Time-Variant Hydraulic-Property Package was developed to accommodate temporal changes in inter-node conductance, hydraulic conductivity, the top and bottom elevations of model layers, and the primary and secondary storage coefficients. The package allows hydraulic properties to be modified step-wise from one stress period to the next. The Time-Variant Hydraulic-Property Package does not alter the formulation of the finite-difference equations in MODFLOW-96 (Harbaugh and McDonald, 1996). Because the package is not a complex modification of MODFLOW, documentation in this appendix is the minimum amount required to implement the package.

\section{Input Instructions}

Input for the Time-Variant Hydraulic-Property Package is read from the unit in IUNIT(21) specified in the basic package input (see McDonald and Harbaugh, 1988, chap. 4, p. 9-11).

FOR EACH SIMULATION

1. Data: MXVAR

VAR1AL

Format:I10

FOR EACH STRESS PERIOD

2. Data: ITMP

VAR1RP

Format:I10

3a. Data: Layer Row Column ModifierPropertyAction (optional)

Format:I10 I10 I10 F10.0 A10 A78

3b. Data: Lay2 Row2 Col2 flag

Format:I10 I10 I10 10x A88

(input item 3 normally consists of either one record for each time-variant hydraulic-property cell modified or two records to define a cube of time-variant hydraulic-property cells to be modified. If ITMP is zero or less, item 3 is not read) 


\section{Explanation of Fields Used in Input Instructions}

MXVAR is the maximum number of time-variant hydraulic-property cells to be modified.

ITMP is a flag.

If $I T M P<=0$, hydraulic-property data from a previous stress period will be reused and input from item 3 will not be read.

If ITMP $>0, \quad$ it is the number of records of hydraulic-property data that will be read for the current stress period.

Layer is the layer number of the cell affected by the modification of the hydraulic property.

Row is the row number of the cell affected by the modification of the hydraulic property.

Column is the column number of the cell affected by the modification of the hydraulic property.

Modifier is the multiplier or replacement value of the hydraulic property to be modified.

Property identifies which hydraulic property is to be modified. The properties are identified by the keys listed in table E1.

Action specifies if the hydraulic property is to be multiplied or replaced by the modifier. If the string "REPLACE" (case insensitive) is detected, the modifier will replace the old value. Otherwise, the old value will be multiplied by the modifier.

Lay2 is the far layer of the cube of cells to be affected by the modification of the hydraulic property.

Row2 is the far row of the cube of cells to be affected by the modification of the hydraulic property.

Col2 is the far column of the cube of cells to be affected by the modification of the hydraulic property.

flag signals this is line $3 \mathrm{~b}$ if the string "CUBE" (case insensitive) is detected. 
Table E1. Hydraulic properties modified by VAR1 and the property keys that identify the properties to be modified

\begin{tabular}{|c|c|c|}
\hline Hydraulic property & Key ${ }^{1}$ & MODFLOW array ${ }^{2}$ \\
\hline Inter-node conductance along rows ${ }^{3}$ & $\underline{\mathrm{X}}$-conductance & $\mathrm{CR}$ \\
\hline Inter-node conductance along columns ${ }^{3}$ & $\underline{Y}$-conductance & $\mathrm{CC}$ \\
\hline Inter-node conductance between layers ${ }^{3}$ & $\underline{Z}$-conductance & $\mathrm{CV}$ \\
\hline $\begin{array}{l}\text { Inter-node conductances along rows and } \\
\qquad \text { columns }^{3}\end{array}$ & $\underline{\mathrm{XY}}$-conductance & $\mathrm{CR}, \mathrm{CC}$ \\
\hline $\begin{array}{l}\text { Inter-node conductances along columns } \\
\text { and between layers }{ }^{3}\end{array}$ & $\underline{\text { YZ-conductance }}$ & $\mathrm{CC}, \mathrm{CV}$ \\
\hline $\begin{array}{l}\text { Inter-node conductances along rows } \\
\text { and between layers }{ }^{3}\end{array}$ & $\underline{\mathrm{XZ}}$-conductance & $\mathrm{CR}, \mathrm{CV}$ \\
\hline $\begin{array}{l}\text { Inter-node conductances along rows and } \\
\text { columns and between layers }{ }^{3}\end{array}$ & $\underline{\mathrm{XYZ}}$-conductance & $\mathrm{CR}, \mathrm{CC}, \mathrm{CV}$ \\
\hline Hydraulic conductivity of layer 1 & $\underline{\mathrm{HY}}$ d. conductivity & HY \\
\hline Elevation of the layer top & $\underline{\text { TOP of layer }}$ & TOP \\
\hline Elevation of the layer bottom & BOTtom of layer & BOT \\
\hline Primary storage coefficient & $\underline{\mathrm{SC} 1}$ & $\mathrm{SC} 1$ \\
\hline Secondary storage coefficient & $\underline{\mathrm{SC} 2}$ & $\mathrm{SC} 2$ \\
\hline
\end{tabular}

${ }^{1}$ Only the underlined fragment of the key is needed.

${ }^{2}$ See McDonald and Harbaugh (1988) for further description of the arrays.

${ }^{3}$ Inter-node conductances modified by the X-conductance, Y-conductance, and Z-conductance keys when applied to the $<\mathrm{i}, \mathrm{j}, \mathrm{k}>$ node are shown below:

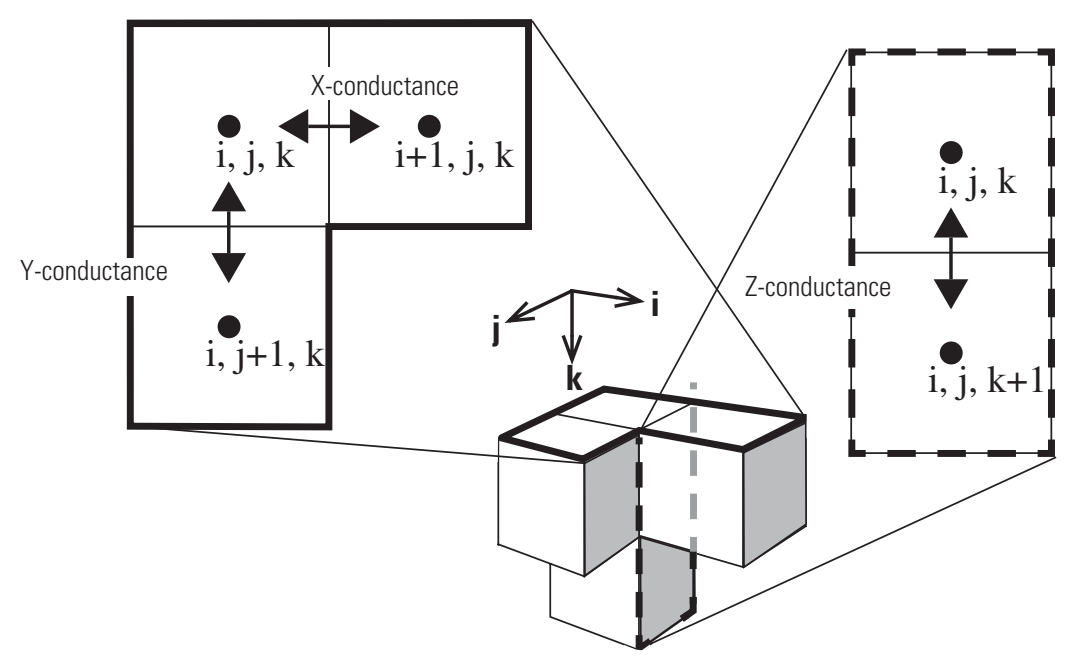



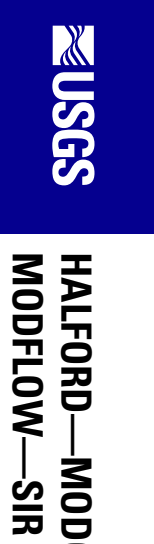

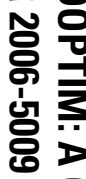

몽

음

롤.

공

ํㅗㅇ

혹

목

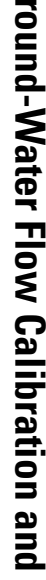

목

章 